\title{
ON 3-DIMENSIONAL HOMOTOPY QUANTUM FIELD THEORY III: COMPARISON OF TWO APPROACHES
}

\author{
VLADIMIR TURAEV AND ALEXIS VIRELIZIER
}

\begin{abstract}
Let $G$ be a discrete group and $\mathcal{C}$ be an additive spherical $G$-fusion category. We prove that the state sum 3-dimensional HQFT derived from $\mathcal{C}$ is isomorphic to the surgery 3-dimensional HQFT derived from the $G$-center of $\mathcal{C}$.
\end{abstract}

\section{INTRODUCTION}

Homotopy Quantum Field Theories (HQFTs) introduced in Tu2 generalize Topological Quantum Field Theories to manifolds and cobordisms endowed with homotopy classes of maps to a fixed target space. We focus here on 3-dimensional HQFTs with target space the (pointed) Eilenberg-MacLane space $K(G, 1)$ where $G$ is a discrete group. Note that the homotopy classes of maps from a manifold $M$ to $K(G, 1)$ bijectively correspond to isomorphism classes of principal $G$-bundles over $M$. In TV2 we defined spherical $G$-fusion categories over a commutative ring $\mathbb{k}$ and showed that such a category $\mathcal{C}$ satisfying a non-degeneracy condition determines a 3 -dimensional HQFT $|\cdot|_{\mathcal{C}}$ over $\mathbb{k}$. The non-degeneracy condition in question requires the $\operatorname{dimension} \operatorname{dim}\left(\mathcal{C}_{1}\right) \in \mathbb{k}$ of the neutral component $\mathcal{C}_{1}$ of $\mathcal{C}$ to be invertible in $\mathbb{k}$. The construction of $|\cdot|_{\mathcal{C}}$ uses Turaev-Viro-Barrett-Westbury-type state sums on skeletons of 3 -manifolds. In TV4, we defined $G$-modular categories over $\mathbb{k}$ and showed that any such category $\mathcal{B}$ endowed with a square root of $\operatorname{dim}\left(\mathcal{B}_{1}\right)$ in $\mathbb{k}$ determines a 3 -dimensional HQFT $\tau_{\mathcal{B}}$ over $\mathbb{k}$. The construction of $\tau_{\mathcal{B}}$ uses Reshetikhin-Turaev-type surgery formulas. The main result of the present paper is an isomorphism between the HQFTs $|\cdot|_{\mathcal{C}}$ and $\tau_{\mathcal{B}}$ provided $\mathcal{B}=\mathcal{Z}_{G}(\mathcal{C})$ is the $G$-center of $\mathcal{C}$ and $\mathbb{k}$ is an algebraically closed field.

To be more specific, consider the cobordism category $\mathrm{Cob}^{G}$ whose objects are $G$-surfaces, that is, pointed closed oriented surfaces endowed with homotopy classes of maps to $K(G, 1)$. Morphisms in $\mathrm{Cob}^{G}$ are represented by $G$-cobordisms defined as compact oriented 3-dimensional cobordisms endowed with homotopy classes of maps to $K(G, 1)$ (for a precise definition of $\mathrm{Cob}^{G}$, see Section 3.1 below). The category $\mathrm{Cob}^{G}$ has a natural structure of a symmetric monoidal category. For a commutative ring $\mathbb{k}$, the category $\operatorname{Mod}_{\mathbb{k}}$ of $\mathbb{k}$-modules and $\mathbb{k}$-homomorphisms is a symmetric monoidal category with monoidal product $\otimes_{\mathbb{k}}$ and unit object $\mathbb{k}$. A 3-dimensional HQFT over $\mathbb{k}$ is a symmetric strong monoidal functor $\mathrm{Cob}^{G} \rightarrow$ $\operatorname{Mod}_{k}$. A precise definition of a 3-dimensional HQFT involves Lagrangian spaces in homology of surfaces and $p_{1}$-structures in cobordisms (see [Tu2]). However, the HQFTs studied in this paper do not depend on this data and we ignore it from now on.

We now state our main results. Consider an additive spherical $G$-fusion category $\mathcal{C}=\oplus_{g \in G} \mathcal{C}_{g}$ over an algebraically closed field $\mathbb{k}$ such that $\operatorname{dim}\left(\mathcal{C}_{1}\right) \neq 0$. Consider the center $\mathcal{Z}_{G}(\mathcal{C})=\oplus_{g \in G} \mathcal{Z}_{g}(\mathcal{C})$ of $\mathcal{C}$ relative to $\mathcal{C}_{1}$ (see GNN for finite $G$ and TV3] for all $G$ ). By [TV3, $\mathcal{Z}_{G}(\mathcal{C})$ is an additive $G$-modular category. Thus $\mathcal{C}$ gives

Date: November 26, 2019.

2000 Mathematics Subject Classification. 57M27, 18D10, 57R56. 
rise to two 3-dimensional HQFTs over $\mathbb{k}$ : the state sum HQFT $|\cdot|_{\mathcal{C}}$ and the surgery HQFT $\tau_{\mathcal{Z}_{G}(\mathcal{C})}$ determined by the square root $\operatorname{dim}\left(\mathcal{C}_{1}\right)$ of $\operatorname{dim}\left(\mathcal{Z}_{1}(\mathcal{C})\right)=\left(\operatorname{dim}\left(\mathcal{C}_{1}\right)\right)^{2}$.

Theorem 1.1. The HQFTs $|\cdot|_{\mathcal{C}}$ and $\tau_{\mathcal{Z}_{G}(\mathcal{C})}$ are isomorphic.

Theorem 1.1 means that one can pick for each $G$-surface $\Sigma$ a $\mathbb{k}$-linear isomorphism of modules $|\Sigma|_{\mathcal{C}} \simeq \tau_{\mathcal{Z}_{G}(\mathcal{C})}(\Sigma)$ so that the resulting family of isomorphisms is compatible with disjoint unions of $G$-surfaces and the action of $G$-cobordisms. For a closed oriented 3-manifold $M$ endowed with a homotopy class of maps to $K(G, 1)$, Theorem 1.1 gives

$$
|M|_{\mathcal{C}}=\tau_{\mathcal{Z}_{G}(\mathcal{C})}(M) \in \mathbb{k} .
$$

For $G=\{1\}$, Theorem 1.1 was established in [TV1] and independently in [Ba].

We prove Theorem 1.1 by first introducing the notion of a graph HQFT over a crossed $G$-graded category (see Section [.1) and then by deducing Theorem 1.1 from the following stronger claim:

Theorem 1.2. Both HQFTs $|\cdot|_{\mathcal{C}}$ and $\tau_{\mathcal{Z}_{G}(\mathcal{C})}$ extend to graph HQFTs over $\mathcal{Z}_{G}(\mathcal{C})$. The resulting graph HQFTs are isomorphic to each other.

Theorem 1.2 is the main result of this paper and is a combination of several claims established in Section 8.2 and of Theorem 8.1 and Theorem 8.2. The extension of the state sum HQFT to a graph HQFT (Theorem 8.1) uses an invariant of colored knotted nets in the 2-dimensional sphere (see Section 91) which plays the role of the familiar $6 j$-symbols. The comparison of the surgery and state sum graph HQFTs (Theorem 8.2) is based on a surgery formula for graph HQFTs via so-called torus vectors (see Section 11). In the appendix, we recall the braided structure of $\mathcal{Z}_{G}(\mathcal{C})$.

We fix throughout the paper a non-zero commutative ring $\mathbb{k}$, a discrete group $G$, and an Eilenberg-MacLane space $\mathbf{X}=K(G, 1)$ with base point $\mathbf{x} \in \mathbf{X}$. Thus, $\mathbf{X}$ is a connected aspherical CW-space and $\pi_{1}(\mathbf{X}, \mathbf{x})=G$.

Acknowledgments. V. Turaev acknowledges support from the NSF grant DMS1664358. A. Virelizier acknowledges support from the Labex CEMPI (ANR-11LABX-0007-01) and from the FNS-ANR grant OChoTop (ANR-18-CE93-0002).

\section{Graded monoidal CATEgories}

We recall the basic notions of the theory of graded and crossed categories referring to [ML], EGNO], Tu2, [TV3]-TV5] for details and proofs.

2.1. Pivotal categories. A pivotal category is a monoidal category $\mathcal{C}=(\mathcal{C}, \otimes, \mathbb{1})$ such that each object $X$ of $\mathcal{C}$ has a dual object $X^{*} \in \mathcal{C}$ and four morphisms

$$
\begin{array}{ll}
\mathrm{ev}_{X}: X^{*} \otimes X \rightarrow \mathbb{1}, & \operatorname{coev}_{X}: \mathbb{1} \rightarrow X \otimes X^{*}, \\
\widetilde{\mathrm{ev}}_{X}: X \otimes X^{*} \rightarrow \mathbb{1}, & \widetilde{\operatorname{coev}_{X}: \mathbb{1} \rightarrow X^{*} \otimes X,}
\end{array}
$$

satisfying several conditions which say, in summary, that the associated left/right dual functors coincide as monoidal functors. In particular, each morphism $f: X \rightarrow$ $Y$ in $\mathcal{C}$ has a dual morphism $f^{*}: Y^{*} \rightarrow X^{*}$ computed by

$$
\begin{aligned}
f^{*} & =\left(\operatorname{ev}_{Y} \otimes \operatorname{id}_{X^{*}}\right)\left(\operatorname{id}_{Y^{*}} \otimes f \otimes \operatorname{id}_{X^{*}}\right)\left(\operatorname{id}_{Y^{*}} \otimes \operatorname{coev}_{X}\right) \\
& =\left(\operatorname{id}_{X^{*}} \otimes \widetilde{\operatorname{ev}}_{Y}\right)\left(\operatorname{id}_{X^{*}} \otimes f \otimes \operatorname{id}_{Y^{*}}\right)\left(\widetilde{\operatorname{coev}_{X}} \otimes \operatorname{id}_{Y^{*}}\right) .
\end{aligned}
$$

We shall omit brackets in monoidal products and suppress the associativity constraints $(X \otimes Y) \otimes Z \cong X \otimes(Y \otimes Z)$, the unitality constraints $X \otimes \mathbb{1} \cong X \cong \mathbb{1} \otimes X$, and the duality constraints $X^{*} \otimes Y^{*} \cong(Y \otimes X)^{*}$ and $\mathbb{1}^{*} \cong \mathbb{1}$. This does not lead 
to ambiguity because, by the Mac Lane coherence theorem, all legitimate ways of inserting these constraints give the same results.

The left and right traces of an endomorphism $f$ of an object $X$ of a pivotal category $\mathcal{C}$ are defined by

$$
\operatorname{tr}_{l}(f)=\operatorname{ev}_{X}\left(\operatorname{id}_{X^{*}} \otimes f\right) \widetilde{\operatorname{coev}_{X}} \quad \text { and } \quad \operatorname{tr}_{r}(f)=\widetilde{\operatorname{ev}}_{X}\left(f \otimes \operatorname{id}_{X^{*}}\right) \operatorname{coev}_{X} .
$$

Both traces take values in the commutative monoid $\operatorname{End}_{\mathcal{C}}(\mathbb{1})$. The left and right dimensions of an object $X \in \mathcal{C}$ are defined by

$$
\operatorname{dim}_{l}(X)=\operatorname{tr}_{l}\left(\operatorname{id}_{X}\right) \in \operatorname{End}_{\mathcal{C}}(\mathbb{1}) \quad \text { and } \quad \operatorname{dim}_{r}(X)=\operatorname{tr}_{r}\left(\operatorname{id}_{X}\right) \in \operatorname{End}_{\mathcal{C}}(\mathbb{1}) .
$$

A spherical category is a pivotal category such that the left and right traces of any endomorphism of any object are equal. In a spherical category, the trace of an endomorphism $f$ and the dimension of an object $X$ are defined by

$$
\operatorname{tr}(f)=\operatorname{tr}_{l}(f)=\operatorname{tr}_{r}(f) \text { and } \operatorname{dim}(X)=\operatorname{dim}_{l}(X)=\operatorname{dim}_{r}(X) .
$$

2.2. Linear categories. A monoidal category $\mathcal{C}$ is $\mathbb{k}$-linear if for all $X, Y \in \mathcal{C}$, the set $\operatorname{Hom}_{\mathcal{C}}(X, Y)$ carries a structure of a left $\mathbb{k}$-module so that both the composition and the monoidal product of morphisms are $\mathbb{k}$-bilinear. Note that then the monoid $\operatorname{End}_{\mathcal{C}}(\mathbb{1})$ is a commutative $\mathbb{k}$-algebra.

In the rest of this section we focus on $\mathbb{k}$-linear pivotal categories. For such a category $\mathcal{C}$, we let $\operatorname{Aut}(\mathcal{C})$ be the category of pivotal strong monoidal $\mathbb{k}$-linear auto-equivalences of $\mathcal{C}$. The objects of the category $\operatorname{Aut}(\mathcal{C})$ are pivotal strong monoidal functors $\mathcal{C} \rightarrow \mathcal{C}$ which are $\mathbb{k}$-linear on the Hom-sets and are equivalences of categories. The morphisms in $\operatorname{Aut}(\mathcal{C})$ are monoidal natural isomorphisms of such functors. Then $\operatorname{Aut}(\mathcal{C})$ is a strict monoidal category with monoidal product being the composition of functors and monoidal unit being the identity endofunctor of $\mathcal{C}$.

2.3. Graded categories. In this paper, by a $G$-graded category $\mathcal{C}$ (over $\mathbb{k}$ ), we mean a $\mathbb{k}$-linear pivotal category endowed with pairwise disjoint full subcategories $\left\{\mathcal{C}_{\alpha}\right\}_{\alpha \in G}$ such that:

(i) if $X \in \mathcal{C}_{\alpha}$ and $Y \in \mathcal{C}_{\beta}$ with $\alpha \neq \beta$, then $\operatorname{Hom}_{\mathcal{C}}(X, Y)=0$;

(ii) if $X \in \mathcal{C}_{\alpha}$ and $Y \in \mathcal{C}_{\beta}$, then $X \otimes Y \in \mathcal{C}_{\alpha \beta}$;

(iii) the unit object $\mathbb{1}$ of $\mathcal{C}$ belongs to $\mathcal{C}_{1}$ where $1 \in G$ is the group unit;

(iv) if $X \in \mathcal{C}_{\alpha}$, then $X^{*} \in \mathcal{C}_{\alpha^{-1}}$.

The category $\mathcal{C}_{1}$ corresponding to $1 \in G$ is called the neutral component of $\mathcal{C}$.

An object $X$ of a $G$-graded category $\mathcal{C}$ is homogeneous if $X \in \mathcal{C}_{\alpha}$ for some $\alpha \in G$. Such an $\alpha$ is then uniquely determined by $X$ and is denoted by $|X|$. We let $\mathcal{C}_{\text {hom }}=\amalg_{\alpha \in G} \mathcal{C}_{\alpha}$ be the full subcategory of homogeneous objects of $\mathcal{C}$. Clearly, $\mathcal{C}_{\text {hom }}$ is itself a $G$-graded category and $\left(\mathcal{C}_{\text {hom }}\right)_{\text {hom }}=\mathcal{C}_{\text {hom }}$. Note that two objects $X \in \mathcal{C}_{\alpha}, Y \in \mathcal{C}_{\beta}$ with $\alpha \neq \beta$ may be isomorphic but then both are zero objects in the sense that $\operatorname{id}_{X}=0$ and $\operatorname{id}_{Y}=0$.

A $G$-graded category $\mathcal{C}$ is additive if any finite (possibly empty) family of objects of $\mathcal{C}$ has a direct sum in $\mathcal{C}$. In this case, we write $\mathcal{C}=\oplus_{\alpha \in G} \mathcal{C}_{\alpha}$.

2.4. Crossed categories. Let $\bar{G}$ be the category whose objects are elements of the group $G$ and morphisms are identities. We view $\bar{G}$ as a strict monoidal category with monoidal product $\alpha \otimes \beta=\beta \alpha$ for all $\alpha, \beta \in G$.

A crossing 11 of a $G$-graded category $\mathcal{C}$ is a strong monoidal functor $\varphi: \bar{G} \rightarrow$ $\operatorname{Aut}(\mathcal{C})$ such that $\varphi_{\alpha}\left(\mathcal{C}_{\beta}\right) \subset \mathcal{C}_{\alpha^{-1} \beta \alpha}$ for all $\alpha, \beta \in G$. The condition that $\varphi_{\alpha}: \mathcal{C} \rightarrow \mathcal{C}$

\footnotetext{
${ }^{1}$ In this paper, crossings correspond to pivotal crossings in TV3, TV4].
} 
is a pivotal strong monoidal functor means that it comes equipped with natural isomorphisms

$$
\begin{aligned}
& \left(\varphi_{\alpha}\right)_{0}: \mathbb{1} \stackrel{\sim}{\longrightarrow} \varphi_{\alpha}(\mathbb{1}), \\
& \left(\varphi_{\alpha}\right)_{2}=\left\{\left(\varphi_{\alpha}\right)_{2}(X, Y): \varphi_{\alpha}(X) \otimes \varphi_{\alpha}(Y) \stackrel{\sim}{\longrightarrow} \varphi_{\alpha}(X \otimes Y)\right\}_{X, Y \in \mathcal{C}}, \\
& \varphi_{\alpha}^{1}=\left\{\varphi_{\alpha}^{1}(X): \varphi_{\alpha}\left(X^{*}\right) \stackrel{\sim}{\longrightarrow}\left(\varphi_{\alpha}(X)\right)^{*}\right\}_{X \in \mathcal{C} .}
\end{aligned}
$$

The condition that the functor $\varphi: \bar{G} \rightarrow \operatorname{Aut}(\mathcal{C})$ is strong monoidal means that it comes equipped with natural isomorphisms

$$
\begin{aligned}
\varphi_{2} & =\left\{\varphi_{2}(\alpha, \beta)=\left\{\varphi_{2}(\alpha, \beta)_{X}: \varphi_{\alpha} \varphi_{\beta}(X) \stackrel{\sim}{\longrightarrow} \varphi_{\beta \alpha}(X)\right\}_{X \in \mathcal{C}}\right\}_{\alpha, \beta \in G}, \\
\varphi_{0} & =\left\{\left(\varphi_{0}\right)_{X}: X \stackrel{\sim}{\longrightarrow} \varphi_{1}(X)\right\}_{X \in \mathcal{C}} .
\end{aligned}
$$

These isomorphisms should satisfy appropriate compatibility conditions, see [TV4].

A $G$-crossed category $(\mathcal{C}, \varphi)$ is a pair consisting of a $G$-graded category $\mathcal{C}$ and a crossing $\varphi$ of $\mathcal{C}$. Then for any $\alpha \in G$ and any integer $n \geq 3$, we have a natural transformation

$$
\left(\varphi_{\alpha}\right)_{n}=\left\{\left(\varphi_{\alpha}\right)_{n}\left(X_{1}, \ldots, X_{n}\right): \varphi_{\alpha}\left(X_{1}\right) \otimes \cdots \otimes \varphi_{\alpha}\left(X_{n}\right) \rightarrow \varphi_{\alpha}\left(X_{1} \otimes \cdots \otimes X_{n}\right)\right\}
$$

where $X_{1}, \ldots, X_{n} \in \mathcal{C}$. It is obtained by composing monoidal products of the transformations $\left(\varphi_{\alpha}\right)_{2}$ and the identity morphisms. For instance, for $n=3$,

$$
\left(\varphi_{\alpha}\right)_{3}\left(X_{1}, X_{2}, X_{3}\right)=\left(\varphi_{\alpha}\right)_{2}\left(X_{1}, X_{2} \otimes X_{3}\right)\left(\operatorname{id}_{\varphi_{\alpha}\left(X_{1}\right)} \otimes\left(\varphi_{\alpha}\right)_{2}\left(X_{2}, X_{3}\right)\right) .
$$

We can also use $\varphi$ to transform certain isomorphisms in $\mathcal{C}$. Namely, for any isomorphism $\psi: X \rightarrow \varphi_{\alpha}(Y)$ with $X, Y \in \mathcal{C}$ and $\alpha \in G$, we let $\psi^{-}: X^{*} \rightarrow \varphi_{\alpha}\left(Y^{*}\right)$ be the composition of the isomorphisms

$$
X^{*} \stackrel{\left(\psi^{-1}\right)^{*}}{\longrightarrow}\left(\varphi_{\alpha}(Y)\right)^{*} \stackrel{\left(\varphi_{\alpha}^{1}(Y)\right)^{-1}}{\longrightarrow} \varphi_{\alpha}\left(Y^{*}\right) .
$$

In this context, we will sometimes write $\psi^{+}$for $\psi$.

2.5. Braided and ribbon graded categories. A $G$-braiding ${ }^{2}$ of a $G$-crossed category $(\mathcal{C}, \varphi)$ is a family of isomorphisms

$$
\tau=\left\{\tau_{X, Y}: X \otimes Y \rightarrow Y \otimes \varphi_{|Y|}(X)\right\}_{X \in \mathcal{C}, Y \in \mathcal{C}_{\mathrm{hom}}}
$$

which is natural in $X, Y$ and satisfies three conditions: two of them generalizing the usual braiding relations in braided monoidal categories and the third condition relating $\tau$ and $\varphi$, see TV4] for details. A $G$-braided category is a $G$-crossed category endowed with a $G$-braiding.

The twist of a $G$-braided category $(\mathcal{C}, \varphi, \tau)$ is the natural isomorphism $\theta=$ $\left\{\theta_{X}\right\}_{X \in \mathcal{C}_{\text {hom }}}$ defined by

$$
\theta_{X}=\left(\operatorname{ev}_{X} \otimes \operatorname{id}_{\varphi_{|X|}(X)}\right)\left(\operatorname{id}_{X} * \otimes \tau_{X, X}\right)\left(\widetilde{\operatorname{coev}_{X}} \otimes \operatorname{id}_{X}\right): X \rightarrow \varphi_{|X|}(X) .
$$

A $G$-ribbon category is a $G$-braided category $(\mathcal{C}, \varphi, \tau)$ whose twist is self-dual in the sense that for all $\alpha \in G$ and all $X \in \mathcal{C}_{\alpha}$,

$$
\left(\theta_{X}\right)^{*}=\left(\varphi_{0}\right)_{X}^{*}\left(\varphi_{2}\left(\alpha^{-1}, \alpha\right)_{X}^{-1}\right)^{*} \varphi_{\alpha^{-1}}^{1}\left(\varphi_{\alpha}(X)\right) \theta_{\varphi_{\alpha}(X)^{*}}
$$

Note that then the neutral component $\mathcal{C}_{1}$ of $\mathcal{C}$ is a ribbon category in the usual sense with braiding

$$
\left\{c_{X, Y}=\left(\operatorname{id}_{Y} \otimes\left(\varphi_{0}\right)_{X}^{-1}\right) \tau_{X, Y}: X \otimes Y \rightarrow Y \otimes X\right\}_{X, Y \in \mathcal{C}_{1}}
$$

and twist

$$
\left\{v_{X}=\left(\varphi_{0}\right)_{X}^{-1} \theta_{X}: X \rightarrow X\right\}_{X \in \mathcal{C}_{1}} .
$$

All $G$-ribbon categories are spherical as pivotal categories (see [TV4, Section 6.3]).

\footnotetext{
${ }^{2}$ In this paper, $G$-braidings correspond to pivotal $G$-braidings in TV3 TV4].
} 
2.6. Fusion graded categories. An object $X$ of a $\mathbb{k}$-linear category is simple if the $\mathbb{k}$-module of the endomorphisms of $X$ is a free $\mathbb{k}$-module of rank 1 with basis $\left\{\operatorname{id}_{X}\right\}$. It is clear that all objects isomorphic to a simple object are simple and (in a pivotal category) the dual of a simple object is simple.

A $G$-fusion category (over $\mathbb{k}$ ) is a $G$-graded category $\mathcal{C}$ (over $\mathbb{k}$ ) such that there is a set $I$ of homogeneous simple objects of $\mathcal{C}$ satisfying the following four conditions:

(a) for each $\alpha \in G$, the set $I_{\alpha} \subset I$ of elements of $I$ belonging to $\mathcal{C}_{\alpha}$ is finite and non-empty;

(b) the unit object $\mathbb{1}$ of $\mathcal{C}$ belongs to $I_{1} \subset I$;

(c) $\operatorname{Hom}_{\mathcal{C}}(i, j)=0$ for any distinct $i, j \in I$;

(d) every object of $\mathcal{C}$ is a direct sum of a finite family of elements of $I$.

The set $I$ is then called a representative set of simple objects. Clearly, $I=\amalg_{\alpha \in G} I_{\alpha}$ and every simple object of $\mathcal{C}$ is isomorphic to precisely one object belonging to $I$.

Let $\mathcal{C}$ be a $G$-fusion category with representative set of simple objects $I$. Condition (b) above implies that the map $\mathbb{k} \rightarrow \operatorname{End}_{\mathcal{C}}(\mathbb{1}), k \mapsto k \mathrm{id}_{\mathbb{1}}$ is a $\mathbb{k}$-algebra isomorphism which we use to identify $\operatorname{End}_{\mathcal{C}}(\mathbb{1})=\mathbb{k}$. Conditions $(\mathrm{c})$ and $(\mathrm{d})$ imply that the Hom-sets in $\mathcal{C}$ are free of finite rank. The neutral component $\mathcal{C}_{1}$ of $\mathcal{C}$ is a fusion (in the usual sense) $\mathbb{k}$-linear pivotal category of dimension

$$
\operatorname{dim}\left(\mathcal{C}_{1}\right)=\sum_{i \in I_{1}} \operatorname{dim}_{l}(i) \operatorname{dim}_{r}(i) \in \operatorname{End}_{\mathcal{C}}(\mathbb{1})=\mathbb{k}
$$

2.7. Modular graded categories. Consider a $G$-ribbon $G$-fusion category $\mathcal{C}$ (over $\mathbb{k}$ ) with representative set of simple objects $I$. For $i, j \in I_{1} \subset I$, set

$$
S_{i, j}=\operatorname{tr}\left(c_{j, i} \circ c_{i, j}: i \otimes j \rightarrow i \otimes j\right) \in \operatorname{End}_{\mathcal{C}}(\mathbb{1})=\mathbb{k}
$$

where $c_{i, j}: i \otimes j \rightarrow j \otimes i$ is the braiding in $\mathcal{C}_{1}$ defined in Section 2.5. The symmetric matrix $S=\left[S_{i, j}\right]_{i, j \in I_{1}}$ is called the $S$-matrix of $\mathcal{C}$. Since each object $i \in I_{1}$ is simple, the twist $v_{i}: i \rightarrow i$ in $\mathcal{C}_{1}$ (see Section 2.5) expands as $v_{i}=\nu_{i} \operatorname{id}_{i}$ with $\nu_{i} \in \mathbb{k}$. Since $v_{i}$ is an isomorphism, $\nu_{i}$ in invertible in $\mathbb{k}$. Set

$$
\Delta_{ \pm}=\sum_{i \in I_{1}} \nu_{i}^{ \pm 1}(\operatorname{dim}(i))^{2} \in \mathbb{k}
$$

where $\operatorname{dim}(i)=\operatorname{dim}_{l}(i)=\operatorname{dim}_{r}(i)$ since such a category $\mathcal{C}$ is spherical.

A $G$-modular category is a $G$-ribbon $G$-fusion category whose $S$-matrix is invertible over the ground ring $\mathbb{k}$. In other words, a $G$-modular category is a $G$-ribbon $G$-fusion category $\mathcal{C}$ whose neutral component $\mathcal{C}_{1}$ is modular in the sense of [Tu1. The properties of modular categories imply that then the elements $\Delta_{ \pm} \in \mathbb{k}$ above are invertible in $\mathbb{k}$ and $\operatorname{dim}\left(\mathcal{C}_{1}\right)=\Delta_{+} \Delta_{-}$, see [Tu1, Formula II.2.4.a]. As a consequence, $\operatorname{dim}\left(\mathcal{C}_{1}\right)$ is invertible in $\mathbb{k}$.

A $G$-modular category $\mathcal{C}$ is anomaly free if $\Delta_{+}=\Delta_{-}$. Then $\Delta=\Delta_{+}=\Delta_{-}$is a square root of $\operatorname{dim}\left(\mathcal{C}_{1}\right)$ called the canonical rank of $\mathcal{C}$.

2.8. The graded center. By $\mathrm{GNN}$, the $G$-center $\mathcal{Z}_{G}(\mathcal{C})$ of a $G$-graded category $\mathcal{C}$ over $\mathbb{k}$ is the category obtained as the (left) center of $\mathcal{C}$ relative to its neutral component $\mathcal{C}_{1} \subset \mathcal{C}$. The objects of $\mathcal{Z}_{G}(\mathcal{C})$ are (left) half braidings of $\mathcal{C}$ relative to $\mathcal{C}_{1}$, that is, pairs $(A, \sigma)$ where $A \in \mathcal{C}$ and

$$
\sigma=\left\{\sigma_{X}: A \otimes X \rightarrow X \otimes A\right\}_{X \in \mathcal{C}_{1}}
$$

is a natural family of isomorphisms satisfying

$$
\sigma_{X \otimes Y}=\left(\operatorname{id}_{X} \otimes \sigma_{Y}\right)\left(\sigma_{X} \otimes \mathrm{id}_{Y}\right)
$$


for all $X, Y \in \mathcal{C}_{1}$. A morphism $(A, \sigma) \rightarrow\left(A^{\prime}, \sigma^{\prime}\right)$ in $\mathcal{Z}_{G}(\mathcal{C})$ is a morphism $f: A \rightarrow A^{\prime}$ in $\mathcal{C}$ such that $\left(\operatorname{id}_{X} \otimes f\right) \sigma_{X}=\sigma_{X}^{\prime}\left(f \otimes \operatorname{id}_{X}\right)$ for all $X \in \mathcal{C}_{1}$. In particular,

$$
\operatorname{Hom}_{\mathcal{Z}_{G}(\mathcal{C})}\left((A, \sigma),\left(A^{\prime}, \sigma^{\prime}\right)\right) \subset \operatorname{Hom}_{\mathcal{C}}\left(A, A^{\prime}\right) \text {. }
$$

The monoidal product of $\mathcal{Z}_{G}(\mathcal{C})$ is defined by

$$
(A, \sigma) \otimes(B, \rho)=\left(A \otimes B,\left(\sigma \otimes \operatorname{id}_{B}\right)\left(\operatorname{id}_{A} \otimes \rho\right)\right)
$$

and the unit object of $\mathcal{Z}_{G}(\mathcal{C})$ is the pair $\mathbb{1}_{\mathcal{Z}_{G}(\mathcal{C})}=\left(\mathbb{1},\left\{\operatorname{id}_{X}\right\}_{X \in \mathcal{C}_{1}}\right)$.

The category $\mathcal{Z}_{G}(\mathcal{C})$ inherits most of the properties of $\mathcal{C}$. The given $\mathbb{k}$-linear structure in $\mathcal{C}$ induces a linear structure in $\mathcal{Z}_{G}(\mathcal{C})$ in the obvious way. The category $\mathcal{Z}_{G}(\mathcal{C})$ is pivotal: the dual of $(A, \sigma) \in \mathcal{Z}_{G}(\mathcal{C})$ is $(A, \sigma)^{*}=\left(A^{*}, \sigma^{\dagger}\right)$, where

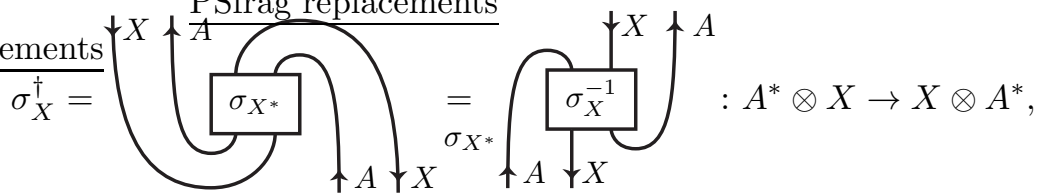

with $\mathrm{ev}_{(A, \sigma)}=\mathrm{ev}_{A}, \operatorname{coev}_{(A, \sigma)}=\operatorname{coev}_{A}, \widetilde{\mathrm{ev}}_{(A, \sigma)}=\widetilde{\mathrm{ev}}_{A}, \widetilde{\operatorname{covv}}_{(A, \sigma)}=\widetilde{\operatorname{coev}}_{A}$. Note that the traces of morphisms and dimensions of objects in $\mathcal{Z}_{G}(\mathcal{C})$ are the same as in $\mathcal{C}$ through the inclusion $\operatorname{End}_{\mathcal{Z}_{G}(\mathcal{C})}\left(\mathbb{1}_{\mathcal{Z}_{G}(\mathcal{C})}\right) \subset \operatorname{End}_{\mathcal{C}}(\mathbb{1})$. The category $\mathcal{Z}_{G}(\mathcal{C})$ is $G$-graded: for $\alpha \in G$, the component $\mathcal{Z}_{\alpha}(\mathcal{C}) \subset \mathcal{Z}_{G}(\mathcal{C})$ is the full subcategory of $\mathcal{Z}_{G}(\mathcal{C})$ formed by the half braidings $(A, \sigma)$ as above with $A \in \mathcal{C}_{\alpha}$. In particular, $\mathcal{Z}_{1}(\mathcal{C})$ is the usual Drinfeld-Joyal-Street center of $\mathcal{C}_{1}$. If $\mathcal{C}$ is additive, then so is its $G$-center, i.e., $\mathcal{Z}_{G}(\mathcal{C})=\oplus_{\alpha \in G} \mathcal{Z}_{\alpha}(\mathcal{C})$.

The forgetful functor $\mathcal{U}: \mathcal{Z}_{G}(\mathcal{C}) \rightarrow \mathcal{C}$ carries $(A, \sigma)$ to $A$ and acts in the obvious way on the morphisms. This functor is strict monoidal, strict pivotal, $\mathbb{k}$-linear, and reflects isomorphisms, meaning that a morphism in $\mathcal{Z}_{G}(\mathcal{C})$ carried to an isomorphism in $\mathcal{C}$ is itself an isomorphism.

If $\mathcal{C}$ is a $G$-fusion category over a field, then $\mathcal{Z}_{G}(\mathcal{C})$ has a canonical structure of a $G$-braided category (see [TV3, Theorem 4.1]). Furthermore, if $\mathcal{C}$ is spherical, then $\mathcal{Z}_{G}(\mathcal{C})$ is $G$-ribbon (see [TV3, Lemma 5.2]). For completeness, we recall in Appendix $\AA$ the construction of the crossing and $G$-braiding of $\mathcal{Z}_{G}(\mathcal{C})$ as well as the computation of the twist of $\mathcal{Z}_{G}(\mathcal{C})$.

By [TV3, Theorem 5.1] and [TV5, Theorem 5.4], if $\mathcal{C}$ is an additive spherical $G$-fusion category over an algebraically closed field such that $\operatorname{dim}\left(\mathcal{C}_{1}\right) \neq 0$, then $\mathcal{Z}_{G}(\mathcal{C})$ is an anomaly free $G$-modular category with canonical rank $\operatorname{dim}\left(\mathcal{C}_{1}\right)$.

\section{Three-Dimensional HQFTs}

We recall the definition of a 3 -dimensional HQFT with target $\mathbf{X}=K(G, 1)$.

3.1. The category $\mathrm{Cob}^{G}$. For an integer $n \geq 0$, by an $n$-manifold, we mean a smooth $n$-dimensional manifold with boundary (possibly, empty). By convention, the empty set $\emptyset$ is considered to be an $n$-manifold for all $n$. The boundary $\partial M$ of an $n$-manifold $M$ is an $(n-1)$-manifold and $\partial(\partial M)=\emptyset$. If $M$ is oriented, then $\partial M$ is oriented so that at any point of $\partial M$, the orientation of $M$ is given by a direction away from $M$ followed by the orientation of $\partial M$. Given an oriented manifold $M$, we let $-M$ be the same manifold with opposite orientation. Clearly, $\partial(-M)=-\partial M$. A closed manifold is a compact manifold with empty boundary.

By a surface we mean a 2-manifold. A surface $\Sigma$ is pointed if every connected component of $\Sigma$ is endowed with a base point. The set of base points of $\Sigma$ is denoted by $\Sigma$. A $G$-surface is a pair consisting of a pointed closed oriented surface $\Sigma$ and a homotopy class $g$ of maps $\left(\Sigma, \Sigma_{\mathbf{0}}\right) \rightarrow(\mathbf{X}, \mathbf{x})$. Reversing orientation in $\Sigma$, we obtain the opposite $G$-surface $-\Sigma=(-\Sigma, g)$. The empty set $\emptyset$ is considered to be a $G$ surface with $\emptyset \bullet=\emptyset$ and $-\emptyset=\emptyset$. The disjoint union of a finite family of $G$-surfaces 
is a $G$-surface in the obvious way. An isomorphism of $G$-surfaces $(\Sigma, g) \rightarrow\left(\Sigma^{\prime}, g^{\prime}\right)$ is an orientation-preserving diffeomorphism $f: \Sigma \rightarrow \Sigma^{\prime}$ such that $f\left(\Sigma_{\bullet}\right)=\Sigma_{\bullet}^{\prime}$ and $g=g^{\prime} f$ as homotopy classes of maps $\left(\Sigma, \Sigma_{\bullet}\right) \rightarrow(\mathbf{X}, \mathbf{x})$.

We define a category $\mathrm{Cob}^{G}$ following [TV2]. The objects of $\mathrm{Cob}^{G}$ are $G$-surfaces. For $G$-surfaces $\Sigma_{0}, \Sigma_{1}$, a morphism $\Sigma_{0} \rightarrow \Sigma_{1}$ in $\mathrm{Cob}^{G}$ is represented by a triple $(M, g, h)$ consisting of a compact oriented 3-manifold $M$ with pointed boundary, a homotopy class $g$ of maps $\left(M,(\partial M)_{\bullet}\right) \rightarrow(\mathbf{X}, \mathbf{x})$, and an isomorphism of $G$-surfaces

$$
h:\left(-\Sigma_{0}\right) \sqcup \Sigma_{1} \rightarrow\left(\partial M,\left.g\right|_{\partial M}\right) .
$$

Such triples $(M, g, h)$ are called $G$-cobordisms between $\Sigma_{0}$ and $\Sigma_{1}$. Two $G$-cobordisms $(M, g, h),\left(M^{\prime}, g^{\prime}, h^{\prime}\right)$ between $\Sigma_{0}$ and $\Sigma_{1}$ represent the same morphism $\Sigma_{0} \rightarrow \Sigma_{1}$ if there is an orientation-preserving diffeomorphism $f: M \rightarrow M^{\prime}$ such that $f\left((\partial M)_{\bullet}\right)=$ $\left(\partial M^{\prime}\right)_{\bullet}, g=g^{\prime} f$, and $h^{\prime}=f h$. Composition of morphisms in $\mathrm{Cob}^{G}$ is defined via the obvious gluing of cobordisms. The identity morphism of a $G$-surface $\Sigma$ is represented by the cylinder $\Sigma \times[0,1]$ endowed with the standard identification of its boundary with $(-\Sigma) \sqcup \Sigma$. The morphisms $\emptyset \rightarrow \emptyset$ in $\mathrm{Cob}^{G}$ are represented by closed 3-dimensional $G$-manifolds, that is, by pairs $(M, g)$ consisting of a closed oriented 3 -manifold $M$ and a homotopy class of maps $g: M \rightarrow \mathbf{X}$.

The category $\mathrm{Cob}^{G}$ with monoidal product induced by disjoint union and with unit object $\emptyset$ is a symmetric monoidal category.

3.2. HQFTs. Let $\operatorname{Mod}_{\mathbb{k}}$ be the symmetric monoidal category of $\mathbb{k}$-modules and $\mathbb{k}$-linear homomorphisms. A 3-dimensional HQFT over $\mathbb{k}$ with target $\mathbf{X}=K(G, 1)$ is a symmetric strong monoidal functor

$$
Z: \mathrm{Cob}^{G} \rightarrow \operatorname{Mod}_{\mathbb{k}} \text {. }
$$

Such a functor carries the following data: a $\mathbb{k}$-module $Z(\Sigma)$ for every $G$-surface $\Sigma$, a $\mathbb{k}$-linear homomorphism $Z(M): Z\left(\Sigma_{0}\right) \rightarrow Z\left(\Sigma_{1}\right)$ for every $G$-cobordism $M: \Sigma_{0} \rightarrow$ $\Sigma_{1}$, a $\mathbb{k}$-linear isomorphism $Z_{0}: \mathbb{k} \simeq Z(\emptyset)$, and $\mathbb{k}$-linear isomorphisms

$$
Z_{2}\left(\Sigma, \Sigma^{\prime}\right): Z(\Sigma) \otimes_{\mathbb{k}} Z\left(\Sigma^{\prime}\right) \simeq Z\left(\Sigma \sqcup \Sigma^{\prime}\right)
$$

for any $G$-surfaces $\Sigma, \Sigma^{\prime}$. This data should satisfy the usual compatibility axioms of a monoidal functor. Two 3-dimensional HQFTs are isomorphic if they are isomorphic as monoidal functors.

A 3-dimensional HQFT $Z$ can be applied to the morphism $\emptyset \rightarrow \emptyset$ in $\mathrm{Cob}^{G}$ represented by a closed 3 -dimensional $G$-manifold $(M, g)$. The resulting endomorphism of $Z(\emptyset) \simeq \mathbb{k}$ is multiplication by an element of $\mathbb{k}$. This element is denoted by $Z(M, g)$ and is a homeomorphism invariant of the pair $(M, g)$. It is clear that isomorphic HQFTs yield the same invariants of closed 3-dimensional $G$-manifolds.

\section{Colored G-Graphs}

In this section, $M$ is a compact oriented 3 -manifold with pointed boundary and $\mathcal{B}$ is a $G$-crossed category over $\mathbb{k}$ (see Section 2.4). We discuss $G$-graphs in $M$ and their $\mathcal{B}$-colorings. Our definitions and results are parallel to those of [TV4] where we studied $G$-graphs in $\mathbb{R}^{3}$.

4.1. Pointed ribbon graphs. We recall the notion of a ribbon graph following Tu1, TV5]. We restrict ourselves to ribbon graphs formed from arcs and coupons and having no circle components. An arc is an oriented segment, i.e., an oriented 1-manifold homeomorphic to $[0,1]$. A coupon is a rectangle with a distinguished side called the bottom base; the opposite side being the top base. A ribbon graph $\Omega$ in $M$ is a union of a finite number of arcs and coupons embedded in $M$ and called the strata of $\Omega$. The strata must satisfy the following two conditions: 
(i) they are disjoint except that some endpoints of the arcs may lie on the bases of the coupons; all other endpoints of the arcs lie in $\partial M \backslash(\partial M)$ • and form $\Omega \cap \partial M$;

(ii) $\Omega$ carries a continuous field of tangent directions in $M$ (the framing) which is transversal to all strata and tangent to $\partial M$ on $\Omega \cap \partial M$.

We provide all coupons of a ribbon graph $\Omega \subset M$ with the orientation which together with the framing of $\Omega$ determine the orientation of $M$ opposite to the given one.

A ribbon graph $\Omega$ in $M$ is pointed if every closed connected component $C$ of $M$ such that $C \cap \Omega \neq \emptyset$ is equipped with a base point lying in $C \backslash \Omega$. The set of these base points is denoted by $\Omega_{\bullet}$. Clearly, the sets $(\partial M)_{\bullet}$ and $\Omega_{\bullet}$ are disjoint, lie in $M \backslash \Omega$, and their union meets every component of $M$ encountering $\Omega$ in at least one point. For example, the empty ribbon graph $\emptyset$ in $M$ is pointed with $\emptyset_{\bullet}=\emptyset$.

4.2. Tracks and detours. Let $\Omega \subset M$ be a pointed ribbon graph. Slightly pushing $\Omega$ along the framing, we obtain a parallel copy $\widetilde{\Omega} \subset M \backslash \Omega$ of $\Omega$ which is also a ribbon graph, see the following picture (in this and the next pictures the orientation of $M$ is right-handed):

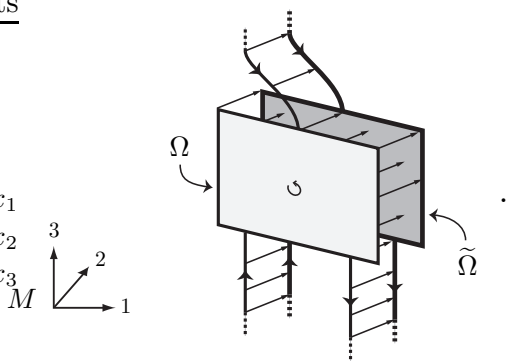

Each stratum $e$ of $\Omega$ yields a stratum $\widetilde{e}$ of $\widetilde{\Omega}$ in the obvious way. A track of $e$ is a homotopy class of paths in $M \backslash \Omega$ leading from a point of $(\partial M) \bullet \cup \Omega$ • to $\tilde{e}$. For a track $\gamma$ of $e$, we let $\mu_{\gamma} \in \pi_{1}(M \backslash \Omega, \gamma(0))$ be the homotopy class of the loop $\gamma l_{e} \gamma^{-1}$, where $l_{e}$ is a small loop in $M \backslash \Omega$ based at the endpoint $\gamma(1)$ of $\gamma$ and encircling $e$ as in the following picture (in particular, if $e$ is an arc then its linking number with $l_{e}$ is -1$)$ :
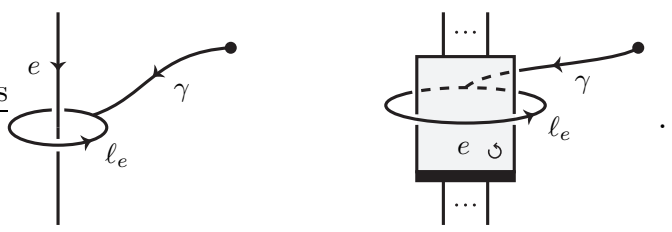

Here and in the sequel, the bottom bases of coupons are drawn boldface.

A detour in $M \backslash \Omega$ is a homotopy class of paths in $M \backslash \Omega$ with endpoints in $(\partial M)_{\bullet} \cup \Omega_{\bullet}$. In particular, the homotopy class of a constant path in a point of $(\partial M)_{\bullet} \cup \Omega_{\bullet}$ is a detour called the constant detour. If a detour $\beta$ in $M \backslash \Omega$ is composable with a track $\gamma$ of a stratum of $\Omega$ (i.e., if $\beta$ ends in the starting point $\gamma(0)$ of $\gamma)$, then $\beta \gamma$ is a track of the same stratum and $\mu_{\beta \gamma}=\beta \mu_{\gamma} \beta^{-1}$. It follows from our definitions that every detour in $M \backslash \Omega$ either has both endpoints in $(\partial M)$. or is a homotopy class of loops based at a point of $\Omega_{\bullet}$.

4.3. $G$-graphs. A $G$-graph is a triple $(M, \Omega, g)$ where $\Omega$ is a pointed ribbon graph in $M$ and $g$ is a homotopy class of maps

$$
\left(M \backslash \Omega,(\partial M) \bullet \cup \Omega_{\bullet}\right) \rightarrow(\mathbf{X}, \mathbf{x}) .
$$

Clearly, $g$ carries any detour in $M \backslash \Omega$ into a homotopy class of loops in $\mathbf{X}$ based at $\mathbf{x}$. The corresponding element of $G=\pi_{1}(\mathbf{X}, \mathbf{x})$ will be denoted by the same letter 
as the detour itself. Similarly, for any track $\gamma$ of a stratum of $\Omega$, the homotopy class of loops $\mu_{\gamma}$ (see Section 4.2) is carried by $g$ into an element of $G$ denoted again by $\mu_{\gamma}$.

For brevity, we will often drop the symbol $g$ from the notation for a $G$-graph $(M, \Omega, g)$ and denote this $G$-graph by $(M, \Omega)$.

4.4. Precolorings. A $\mathcal{B}$-precoloring $u$ of a $G$-graph $(M, \Omega)$ comprises two functions. The first function assigns to every track $\gamma$ of an arc of $\Omega$ an object $u_{\gamma} \in \mathcal{B}_{\mu_{\gamma}}$ called the color of $\gamma$ (see Section 4.2 for the definition of $\mu_{\gamma}$ ). The second function assigns to every pair $(\beta, \gamma)$, where $\beta$ is a detour in $M \backslash \Omega$ and $\gamma$ is a track of an arc of $\Omega$ which is composable with $\beta$, an isomorphism

$$
u_{\beta, \gamma}: u_{\beta \gamma} \rightarrow \varphi_{\beta-1}\left(u_{\gamma}\right)
$$

These functions must satisfy the following two conditions (where $\varphi$ denotes the crossing of $\mathcal{B})$ :

(i) If $\beta$ is the constant detour at the starting point of a track $\gamma$ of an arc of $\Omega$, then

$$
u_{\beta, \gamma}=\left(\varphi_{0}\right)_{u_{\gamma}}: u_{\gamma} \rightarrow \varphi_{1}\left(u_{\gamma}\right)
$$

(ii) For any composable detours $\beta, \delta$ in $M \backslash \Omega$ and any track $\gamma$ of an arc of $\Omega$ which is composable with $\delta$, the following diagram commutes:

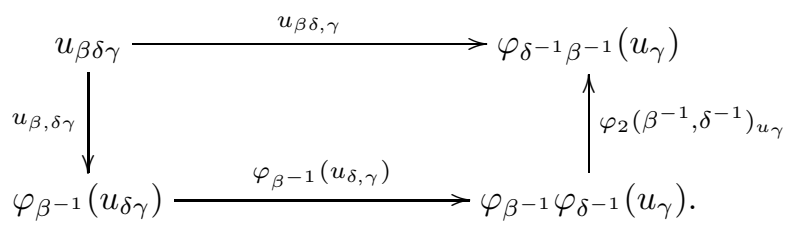

A $\mathcal{B}$-precolored $G$-graph is a $G$-graph endowed with a $\mathcal{B}$-precoloring. Disjoint unions of $\mathcal{B}$-precolored $G$-graphs are $\mathcal{B}$-precolored $G$-graphs in the obvious way.

4.5. Colorings. A coupon-coloring of a $\mathcal{B}$-precolored $G$-graph $((M, \Omega), u)$ is a function which assigns to each track $\gamma$ of a coupon $c$ of $\Omega$ a morphism $v_{\gamma}$ in the category $\mathcal{B}_{\mu_{\gamma}} \subset \mathcal{B}$. To state our requirements on $v_{\gamma}$, we need more terminology. The inputs (respectively, outputs) of $c$ are the endpoints of the arcs of $\Omega$ lying on the bottom (respectively, top) base of $c$. The direction of the bottom base induced by the orientation of $c$ determines an order in the set of the inputs. Let $m \geq 0$ be the number of inputs of $c$ and let $e_{k}$ be the arc of $\Omega$ incident to the $k$-th input for $k=1, \ldots, m$. Set $\varepsilon_{k}=+$ if $e_{k}$ is directed out of $c$ at the $k$-th input and $\varepsilon_{k}=-$ otherwise. Similarly, the direction of the top base of $c$ induced by the opposite orientation of $c$ determines an order in the set of the outputs. Let $n \geq 0$ be the number of outputs of $c$ and let $e^{l}$ be the arc of $\Omega$ incident to the $l$-th output for $l=1, \ldots, n$. Set $\varepsilon^{l}=+$ if $e^{l}$ is directed into $c$ at the $l$-th output and $\varepsilon^{l}=-$ otherwise. Recall the parallel copy $\widetilde{c} \subset \widetilde{\Omega}$ of $c$ (see Section 4.2). Let $\gamma$ be a track of $c$. Composing $\gamma$ with a path in $\widetilde{c}$ leading to the $k$-th input, we obtain a track $\rho_{k}$ of $e_{k}$. Composing $\gamma$ with a path in $\widetilde{c}$ leading to the $l$-th output, we obtain a track $\rho^{l}$ of $e^{l}$. Clearly,

$$
\mu_{\gamma}=\mu_{\rho_{1}}^{\varepsilon_{1}} \cdots \mu_{\rho_{m}}^{\varepsilon_{m}}=\mu_{\rho^{1}}^{\varepsilon^{1}} \cdots \mu_{\rho^{n}}^{\varepsilon^{n}} \in \pi_{1}(M \backslash \Omega, \gamma(0))
$$

where $\gamma(0) \in(\partial M) \bullet \cup \Omega_{\bullet}$ is the starting point of $\gamma$. Set

$$
u_{\gamma}=\bigotimes_{k=1}^{m} u_{\rho_{k}}^{\varepsilon_{k}} \quad \text { and } \quad u^{\gamma}=\bigotimes_{l=1}^{n} u_{\rho^{l}}^{\varepsilon^{l}},
$$

where $X^{+}=X$ and $X^{-}=X^{*}$ for any object $X \in \mathcal{B}$. We require that:

(i) $v_{\gamma} \in \operatorname{Hom}_{\mathcal{B}}\left(u_{\gamma}, u^{\gamma}\right)$ for any track $\gamma$ of any coupon of $\Omega$; 
(ii) for any detour $\beta$ in $M \backslash \Omega$ composable with a track $\gamma$ of a coupon of $\Omega$, the following diagram (using the notation above for the inputs and outputs of the coupon) commutes:

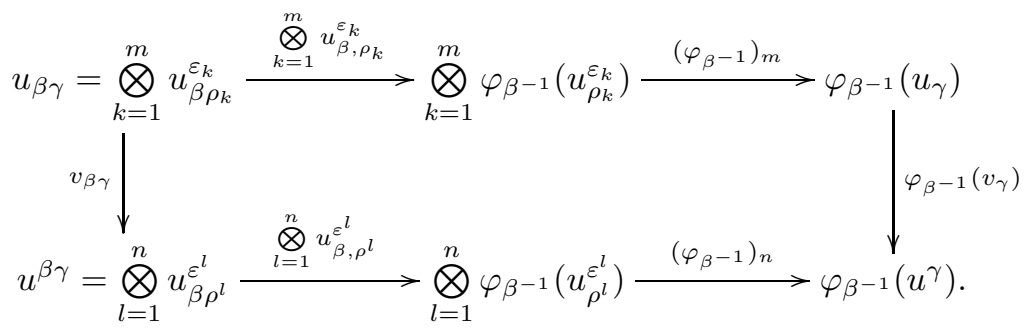

Here $u_{\beta, \gamma}^{+}=u_{\beta, \gamma}$ while $u_{\beta, \gamma}^{-}: u_{\beta \gamma}^{*} \rightarrow \varphi_{\beta^{-1}}\left(u_{\gamma}^{*}\right)$ and $\left(\varphi_{\beta^{-1}}\right)_{m}$ are defined in Section 2.4.

For $m=n=1$ and $\varepsilon_{1}=\varepsilon^{1}=+$, the diagram (2) simplifies to

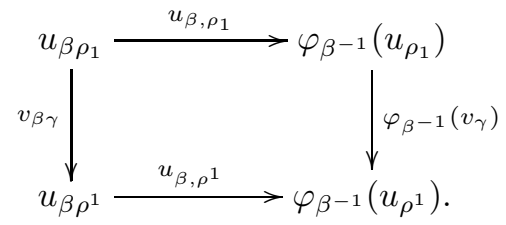

A $\mathcal{B}$-coloring of a $G$-graph $(M, \Omega)$ is a pair $(u, v)$ where $u$ is a $\mathcal{B}$-precoloring of $(M, \Omega)$ and $v$ is a coupon-coloring of $((M, \Omega), u)$. A $\mathcal{B}$-colored $G$-graph is a $G$-graph endowed with a $\mathcal{B}$-coloring. Disjoint unions of $\mathcal{B}$-colored $G$-graphs are $\mathcal{B}$-colored $G$-graphs in the obvious way.

4.6. Stabilization and conjugation. We define two operations on a $\mathcal{B}$-colored $G$-graphs called stabilization and conjugation. To stabilize a $\mathcal{B}$-colored $G$-graph $(M, \Omega, g)$, we insert a small coupon $c$ in the middle of an arc of $\Omega$, keeping the rest of the data. We take as the bottom base of $c$ one of its sides incident to the arc. We insert $c$ so that it is transverse to the framing $\nu$ at the middle point of the arc, and provide $c$ with the constant framing equal to $\nu$ :

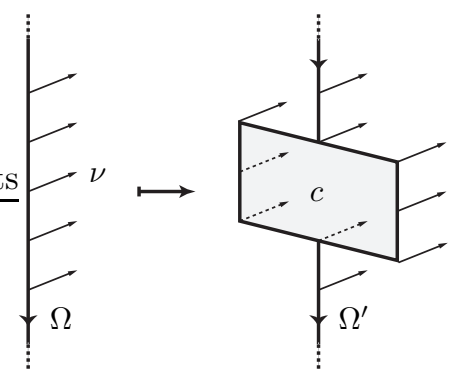

This gives a $G$-graph $\left(M, \Omega^{\prime}, g^{\prime}\right)$, where the class of maps $g^{\prime}$ is the restriction of $g$ via the inclusion $M \backslash \Omega^{\prime} \subset M \backslash \Omega$. The given $\mathcal{B}$-precoloring $u$ of $\Omega$ restricts to a $\mathcal{B}$-precoloring $u^{\prime}$ of $\Omega^{\prime}$ via the same inclusion. The given coupon-coloring of $\Omega$ similarly restricts to a coupon-coloring of all coupons of $\Omega^{\prime}$ except $c$. We color any track $\gamma^{\prime}$ of $c$ with the identity morphism of the object $u_{\gamma^{\prime}}^{\prime}=\left(u^{\prime}\right)^{\gamma^{\prime}}$. Note that this object is computed by $u_{\gamma}^{\varepsilon}$, where $\gamma$ is the track of the original arc of $\Omega$ determined by $\gamma^{\prime}$ and $\varepsilon=+$ if the arc incident to the bottom base of $c$ is directed out of $c$ while $\varepsilon=-$ otherwise.

The conjugation by any $\kappa \in G$ of a $G$-graph $(M, \Omega, g)$ produces a $G$-graph $(M, \Omega, g)^{\kappa}=\left(M, \Omega, g^{\kappa}\right)$, where $g^{\kappa}$ is the composition of $g$ with the homotopy class of maps $(\mathbf{X}, x) \rightarrow(\mathbf{X}, x)$ representing the endomorphism of $G=\pi_{1}(\mathbf{X}, \mathbf{x})$ carrying 
each $\alpha \in G$ to $\kappa^{-1} \alpha \kappa \in G$. This transformation lifts to $\mathcal{B}$-colored $G$-graphs as follows. The precoloring $u$ of $(M, \Omega, g)$ induces a precoloring $u^{\kappa}$ of $(M, \Omega, g)^{\kappa}$ which carries every track $\gamma$ of an arc of $\Omega$ to $\left(u^{\kappa}\right)_{\gamma}=\varphi_{\kappa}\left(u_{\gamma}\right)$ and carries every pair $(\beta, \gamma)$, where $\beta$ is a detour in $M \backslash \Omega$ and $\gamma$ is a track of an arc of $\Omega$ composable with $\beta$, to the isomorphism $\left(u^{\kappa}\right)_{\beta, \gamma}$ defined as the following composition:

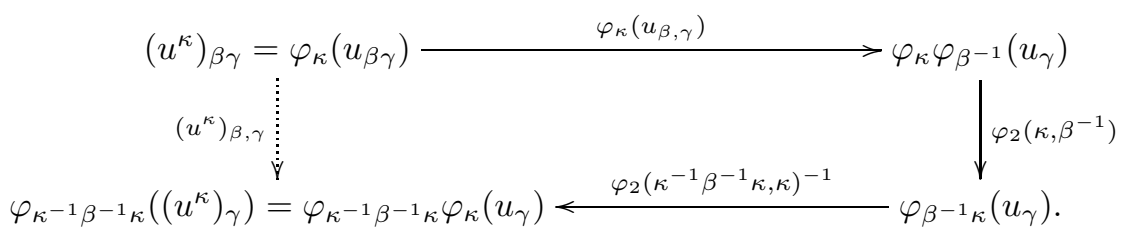

Here, the element of $G=\pi_{1}(\mathbf{X}, \mathbf{x})$ represented by the image under $g$ of the detour $\beta$ is denoted (as above) by the same letter $\beta$, so that the element of $G$ represented by the image under $g^{\kappa}$ of the detour $\beta$ is indeed computed by $\kappa^{-1} \beta \kappa$. The coupon-coloring $v^{\kappa}$ of $\left((M, \Omega, g)^{\kappa}, u^{\kappa}\right)$ is similarly induced by the coupon-coloring $v$ of $((M, \Omega, g), u)$ and the isomorphisms $\left(\varphi_{\kappa}\right)_{n}$ and $\varphi_{\kappa}^{1}$ associated with $\varphi$ (see Section 2.4). More explicitly, if $\gamma$ is a track of a coupon of $\Omega$ and using the notation of Section 4.5 then the morphism $\left(v^{\kappa}\right)_{\gamma}$ is defined as the following composition:

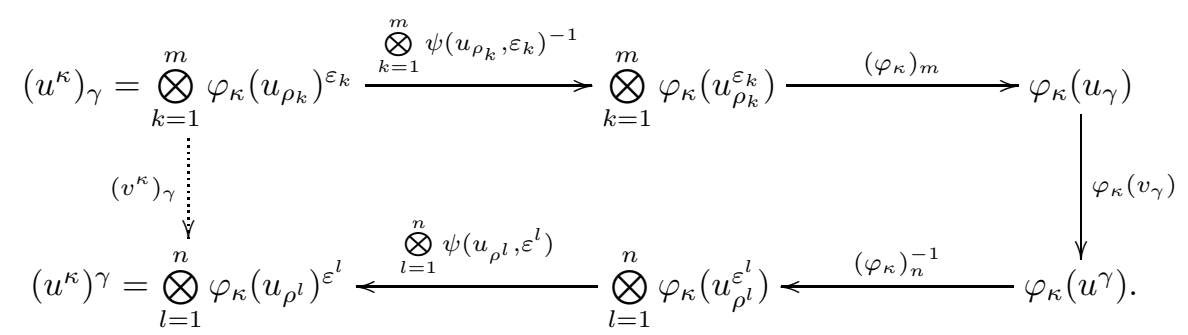

Here, for an object $X \in \mathcal{B}$, we set $\psi(X,+)=\operatorname{id}_{\varphi_{\kappa}(X)}$ and $\psi(X,-)=\varphi_{\kappa}^{1}(X)$.

Two key properties of this transformation will be stated in Section 5.2 .

\section{ISOMORPHISMS AND CONSTRUCTIONS OF COLORINGS}

In this section, $\mathcal{B}$ is a $G$-crossed category over $\mathbb{k}$.

5.1. Isomorphisms of $G$-graphs. An isomorphism between $G$-graphs $(M, \Omega, g)$ and $\left(M^{\prime}, \Omega^{\prime}, g^{\prime}\right)$ is an orientation-preserving diffeomorphism $f: M \rightarrow M^{\prime}$ which carries $(\partial M)_{\bullet}$ onto $\left(\partial M^{\prime}\right)_{\bullet}, \Omega_{\bullet}$ onto $\Omega_{\bullet}^{\prime}$, and $\Omega$ onto $\Omega^{\prime}$ (preserving the strata, the orientation, and the framing) and satisfies

$$
g=g^{\prime} f:\left(M \backslash \Omega,(\partial M) \bullet \cup \Omega_{\bullet}\right) \rightarrow(\mathbf{X}, \mathbf{x}) .
$$

Composing $f$ with a track $\gamma$ of a stratum of $\Omega$, we obtain a track $f \gamma$ of the corresponding stratum of $\Omega^{\prime}$. Similarly, composing $f$ with a detour in $M \backslash \Omega$, we obtain a detour $f \beta$ in $M^{\prime} \backslash \Omega^{\prime}$.

An isomorphism between $\mathcal{B}$-precolored $G$-graphs $((M, \Omega), u)$ and $\left(\left(M^{\prime}, \Omega^{\prime}\right), u^{\prime}\right)$ is a pair $(f, w)$ where $f: M \rightarrow M^{\prime}$ is an isomorphism between the $G$-graphs $(M, \Omega),\left(M^{\prime}, \Omega^{\prime}\right)$ and $w$ is a function which assigns to every track $\gamma$ of an arc of $\Omega$ an isomorphism $w_{\gamma}: u_{\gamma} \rightarrow u_{f \gamma}^{\prime}$ in $\mathcal{B}$ so that for any such $\gamma$ and any detour $\beta$ in $M \backslash \Omega$ composable with $\gamma$, the following diagram commutes:

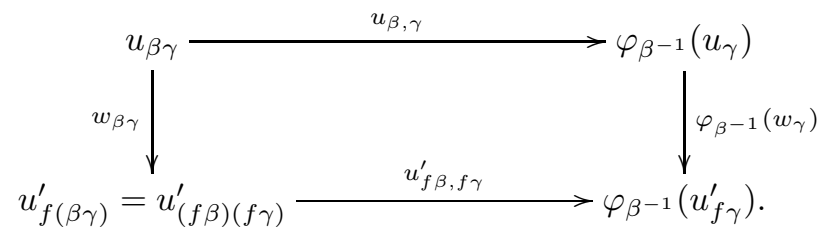


An isomorphism between $\mathcal{B}$-colored $G$-graphs $((M, \Omega), u, v)$ and $\left(\left(M^{\prime}, \Omega^{\prime}\right), u^{\prime}, v^{\prime}\right)$ is an isomorphism $(f, w)$ between the underlying $\mathcal{B}$-precolored $G$-graphs $((M, \Omega), u)$ and $\left(\left(M^{\prime}, \Omega^{\prime}\right), u^{\prime}\right)$ such that for each track $\gamma$ of a coupon of $\Omega$, the diagram

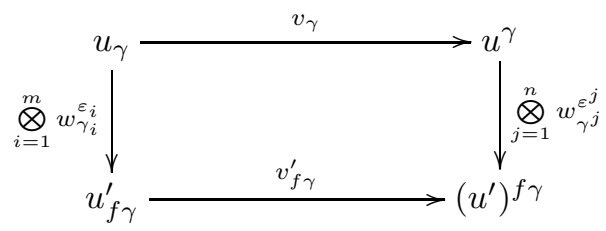

commutes. (Here, we use the same notation as in Section 4.5.) Since the vertical arrows are isomorphisms, the commutativity of this diagram implies that $v^{\prime}$ is uniquely determined by $v$ and the isomorphism $(f, w)$.

5.2. Strong isomorphisms. A strong isomorphism between two $\mathcal{B}$-precolorings $u, u^{\prime}$ of a same $G$-graph $(M, \Omega)$ is a function $w$ which assigns to every track $\gamma$ of an arc of $\Omega$ an isomorphism $w_{\gamma}: u_{\gamma} \rightarrow u_{\gamma}^{\prime}$ in $\mathcal{B}$ such that the pair $\left(\operatorname{id}_{M}: M \rightarrow M, w\right)$ is an isomorphism between the $\mathcal{B}$-precolored $G$-graphs $((M, \Omega), u)$ and $\left((M, \Omega), u^{\prime}\right)$.

A strong isomorphism between two $\mathcal{B}$-colorings $(u, v)$ and $\left(u^{\prime}, v^{\prime}\right)$ of a $G$-graph $(M, \Omega)$ is a strong isomorphism $w$ between the $\mathcal{B}$-precolorings $u, u^{\prime}$ of $(M, \Omega)$ such that the pair $\left(\operatorname{id}_{M}: M \rightarrow M, w\right)$ is an isomorphism between the $\mathcal{B}$-colored $G$-graphs $((M, \Omega), u, v)$ and $\left((M, \Omega), u^{\prime}, v^{\prime}\right)$.

The notion of a strong isomorphism allows us to formulate two key properties of the conjugation of a $\mathcal{B}$-colored $G$-graph $((M, \Omega), u, v)$, see Section 4.6. Namely, the $\mathcal{B}$-colored $G$-graph $((M, \Omega), u, v)^{1}$ obtained via conjugation by $1 \in G$ is strongly isomorphic to the original $\mathcal{B}$-colored $G$-graph $((M, \Omega), u, v)$. Also, for any $\kappa, \kappa^{\prime} \in G$, the $\mathcal{B}$-colored $G$-graphs

$$
((M, \Omega), u, v)^{\kappa \kappa^{\prime}} \text { and } \quad\left(((M, \Omega), u, v)^{\kappa}\right)^{\kappa^{\prime}}
$$

are strongly isomorphic. We leave the proofs of these claims to the reader.

5.3. Systems of tracks. A 1-system of tracks for a $G$-graph $(M, \Omega)$ is a family $\left\{\gamma_{e}\right\}_{e}$ where $e$ runs over the arcs of $\Omega$ and $\gamma_{e}$ is a track of $e$. To exhibit such a system we just pick one track for every arc of $\Omega$ (this is possible since every component of $M$ meeting $\Omega$ also meets $\left.(\partial M) \bullet \cup \Omega_{\bullet}\right)$. An arbitrary family of objects $\left\{u_{e} \in \mathcal{B}_{\mu_{\gamma_{e}}}\right\}_{e}$ determines a $\mathcal{B}$-precoloring $u$ of $(M, \Omega)$ as follows. Each track $\gamma$ of an arc $e$ of $\Omega$ expands uniquely as $\gamma=\alpha \gamma_{e}$ where $\alpha$ is a detour in $M \backslash \Omega$. Set

$$
u_{\gamma}=\varphi_{\alpha^{-1}}\left(u_{e}\right) \in \mathcal{B}_{\alpha \mu_{\gamma_{e}} \alpha^{-1}}=\mathcal{B}_{\mu_{\gamma}} .
$$

Note that $u_{\gamma_{e}}=\varphi_{1}\left(u_{e}\right)$ is canonically isomorphic to $u_{e}$. For any detour $\beta$ in $M \backslash \Omega$ such that $\gamma$ is composable with $\beta$, set

$$
u_{\beta, \gamma}=\left(\varphi_{2}\left(\beta^{-1}, \alpha^{-1}\right)_{u_{e}}\right)^{-1}: u_{\beta \gamma}=\varphi_{\alpha^{-1} \beta^{-1}}\left(u_{e}\right) \rightarrow \varphi_{\beta^{-1}} \varphi_{\alpha^{-1}}\left(u_{e}\right)=\varphi_{\beta^{-1}}\left(u_{\gamma}\right) .
$$

Lemma 5.1. The objects and isomorphisms defined above form a $\mathcal{B}$-precoloring $u$ of $(M, \Omega)$ which only depends (up to strong isomorphism) on the isomorphism classes of the objects $\left\{u_{e}\right\}_{e}$.

Proof. That $u$ is a $\mathcal{B}$-precoloring is a direct consequence of the following axioms of the crossing $\varphi$ (see [TV4]): $\varphi_{2}(1, a)_{X}=\left(\varphi_{0}\right)_{\varphi_{a}(X)}$ and

$$
\varphi_{2}(b a, c)_{X} \varphi_{2}(a, b)_{\varphi_{c}(X)}=\varphi_{2}(a, c b)_{X} \varphi_{a}\left(\varphi_{2}(b, c)_{X}\right)
$$

for all $a, b, c \in G$ and all object $X$ of $\mathcal{B}$.

Next, consider a family of isomorphisms $\left\{\phi_{e}: u_{e} \rightarrow u_{e}^{\prime}\right\}_{e}$ in $\mathcal{B}$ and denote by $u^{\prime}$ the $\mathcal{B}$-precoloring of $(M, \Omega)$ derived as above from the family $\left\{u_{e}^{\prime}\right\}_{e}$. For a track $\gamma$ of an arc $e$ of $\Omega$, expand $\gamma=\alpha \gamma_{e}$ as above and set

$$
w_{\gamma}=\varphi_{\alpha^{-1}}\left(\phi_{e}\right): u_{\gamma}=\varphi_{\alpha^{-1}}\left(u_{e}\right) \rightarrow \varphi_{\alpha^{-1}}\left(u_{e}^{\prime}\right)=u_{\gamma}^{\prime} .
$$


It follows directly from the naturality of $\varphi_{2}$ that the function $\gamma \mapsto w_{\gamma}$ is a strong isomorphism between the $\mathcal{B}$-precolorings $u, u^{\prime}$ of $(M, \Omega)$.

The following lemma shows that this construction yields all $\mathcal{B}$-precolorings of $(M, \Omega)$, at least up to strong isomorphism.

Lemma 5.2. Any $\mathcal{B}$-precoloring $U$ of $(M, \Omega)$ is strongly isomorphic to the $\mathcal{B}$ precoloring $u$ of $(M, \Omega)$ determined by the family of objects $\left\{u_{e}=U_{\gamma_{e}}\right\}_{e}$.

Proof. For a track $\gamma$ of an arc $e$ of $\Omega$, expand $\gamma=\alpha \gamma_{e}$ as above and set

$$
w_{\gamma}=U_{\alpha, \gamma_{e}}: U_{\gamma}=U_{\alpha \gamma_{e}} \rightarrow \varphi_{\alpha^{-1}}\left(U_{\gamma_{e}}\right)=u_{\gamma}
$$

It is easy to check that the function $\gamma \mapsto w_{\gamma}$ is a strong isomorphism between the $\mathcal{B}$-precolorings $U$ and $u$ of $(M, \Omega)$.

A 2-system of tracks for a $G$-graph $(M, \Omega)$ is a family $\left\{\gamma_{c}\right\}_{c}$ where $c$ runs over all coupons of $\Omega$ and $\gamma_{c}$ is a track of $c$. To exhibit such a system we just fix one track for every $c$. Given a $\mathcal{B}$-precoloring $u$ of $(M, \Omega)$, pick a 2 -system of tracks $\left\{\gamma_{c}\right\}_{c}$ for $(M, \Omega)$ and an arbitrary family of morphisms $\left\{v_{c}: u_{\gamma_{c}} \rightarrow u^{\gamma_{c}}\right\}_{c}$ in $\mathcal{B}$. (Here, the objects $u_{\gamma_{c}}$ and $u^{\gamma_{c}}$ are as in Section 4.5). Each track of a coupon $c$ of $\Omega$ expands uniquely as $\beta \gamma_{c}$ where $\beta$ is a detour in $M \backslash \Omega$. Formula (2) with $\gamma=\gamma_{c}$ and $v_{\gamma}=v_{c}$ defines a morphism

$$
v_{\beta \gamma_{c}}: u_{\beta \gamma_{c}} \rightarrow u^{\beta \gamma_{c}}
$$

in $\mathcal{B}$. This yields a coupon-coloring $v$ of $((M, \Omega), u)$ and a $\mathcal{B}$-coloring $(u, v)$ of $(M, \Omega)$. Lemma 5.2 implies that all colorings of $\Omega$ may be obtained in this way, at least up to strong isomorphism.

\section{Colored G-Surfaces}

In this section we introduce markings and colorings of $G$-surfaces. We fix until the end of the section a $G$-crossed category $\mathcal{B}$ over $\mathbb{k}$.

6.1. Marked $G$-surfaces. A point of a surface is marked if it is endowed with a tangent direction and a sign \pm 1 . A marked $G$-surface is a triple consisting of a pointed closed oriented surface $\Sigma$, a finite set of marked points $A \subset \Sigma \backslash \Sigma$, and a homotopy class of maps $g:\left(\Sigma \backslash A, \Sigma_{\bullet}\right) \rightarrow(\mathbf{X}, \mathbf{x})$. For a point $a \in A$, we let $\varepsilon_{a}= \pm 1$ be its sign and let $*_{a} \in \Sigma \backslash A$ be the base point of the connected component of $\Sigma$ containing $a$. Slightly pushing $a$ in the given tangent direction we get a point $\tilde{a} \in \Sigma \backslash A$. A track of $a$ is a homotopy class $\gamma$ of paths leading from $*_{a}$ to $\tilde{a}$ in $\Sigma \backslash A$. We let $\mu_{\gamma} \in \pi_{1}\left(\Sigma \backslash A, *_{a}\right)$ be the element represented the loop $\gamma m_{a}^{\varepsilon_{a}} \gamma^{-1}$, where $m_{a}$ is a small loop in $\Sigma \backslash A$ based in $\tilde{a}$ and encircling $a$ in the direction induced by the orientation of $\Sigma$ (i.e., $m_{a}$ is the boundary of disk embedded in $\Sigma \backslash(A \backslash\{a\})$ containing $a$ in its interior and $\tilde{a}$ in its boundary). Multiplication by loops defines a left action of the group $\pi_{1}\left(\Sigma \backslash A, *_{a}\right)$ on the set of tracks of $a$. Clearly, $\mu_{\beta \gamma}=\beta \mu_{\gamma} \beta^{-1}$ for any $\beta \in \pi_{1}\left(\Sigma \backslash A, *_{a}\right)$. As above, we denote by the same letters the elements of $\pi_{1}\left(\Sigma \backslash A, *_{a}\right)$ and their images under the group homomorphism $\pi_{1}\left(\Sigma \backslash A, *_{a}\right) \rightarrow G$ induced by $g$.

An isomorphism of marked $G$-surfaces $(\Sigma, A, g)$ and $\left(\Sigma^{\prime}, A^{\prime}, g^{\prime}\right)$ is an orientation-preserving diffeomorphism $f: \Sigma \rightarrow \Sigma^{\prime}$ which carries $\Sigma_{\bullet}$ onto $\Sigma_{\bullet}^{\prime}$ and $A$ onto $A^{\prime}$ (preserving the tangent directions and the signs of the marked points) and such that $g=g^{\prime} f$.

For brevity, we will often denote a marked $G$-surface $(\Sigma, A, g)$ by $(\Sigma, A)$ or even by $\Sigma$ suppressing $g$ and $A$. 
6.2. Colored $G$-surfaces. A $\mathcal{B}$-coloring $u$ of a marked $G$-surface $(\Sigma, A)$ comprises two functions. The first function assigns to every track $\gamma$ of any $a \in A$ an object $u_{\gamma} \in \mathcal{B}_{\mu_{\gamma}}$ called the color of $\gamma$. The second function assigns to every track $\gamma$ of any $a \in A$ and to every $\beta \in \pi_{1}\left(\Sigma \backslash A, *_{a}\right)$ an isomorphism

$$
u_{\beta, \gamma}: u_{\beta \gamma} \rightarrow \varphi_{\beta-1}\left(u_{\gamma}\right)
$$

such that:

(i) for every track $\gamma$,

$$
u_{1, \gamma}=\left(\varphi_{0}\right)_{u_{\gamma}}: u_{\gamma} \rightarrow \varphi_{1}\left(u_{\gamma}\right)
$$

(ii) for every track $\gamma$ of any $a \in A$ and for all $\beta, \delta \in \pi_{1}\left(\Sigma \backslash A, *_{a}\right)$, the following diagram commutes:

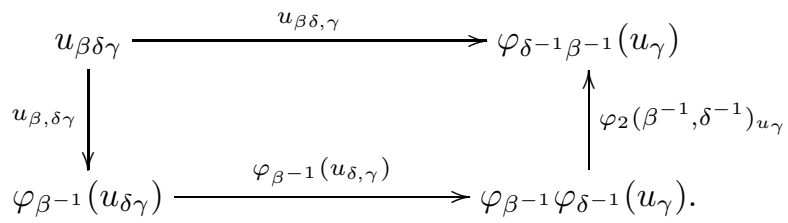

A $\mathcal{B}$-colored $G$-surface is a marked $G$-surface endowed with a $\mathcal{B}$-coloring. Disjoint unions of $\mathcal{B}$-colored $G$-surfaces are $\mathcal{B}$-colored $G$-surfaces in the obvious way. Reversing the orientation of the ambient surface and the signs of all marked points (while keeping the colors of the tracks), we transform a $\mathcal{B}$-colored $G$-surface $\Sigma$ into the opposite $\mathcal{B}$-colored $G$-surface $-\Sigma$.

An isomorphism between $\mathcal{B}$-colored $G$-surfaces $(\Sigma, A, u)$ and $\left(\Sigma^{\prime}, A^{\prime}, u^{\prime}\right)$ is a pair $(f, F)$ consisting of an isomorphism $f: \Sigma \rightarrow \Sigma^{\prime}$ of the underlying marked $G$-surfaces and a function $F$ which assigns to every track $\gamma$ of any $a \in A$ an isomorphism $F_{\gamma}: u_{\gamma} \rightarrow u_{f \gamma}^{\prime}$ in $\mathcal{B}$ such that, for all $\beta \in \pi_{1}\left(\Sigma \backslash A, *_{a}\right)$, the following diagram commutes:

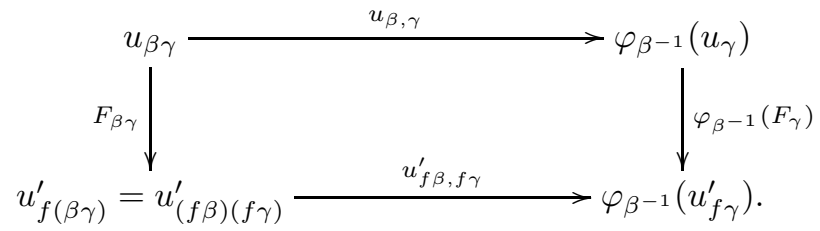

Here $f \gamma$ is the track of $f(a) \in A^{\prime}$ obtained by composing $\gamma$ and $f$.

6.3. Strong isomorphisms and systems of tracks. Let $(\Sigma, A)$ be a marked $G$-surface. A strong isomorphism between two $\mathcal{B}$-colorings $u, u^{\prime}$ of $(\Sigma, A)$ is a function $F$ which assigns to every track $\gamma$ of any $a \in A$ an isomorphism $F_{\gamma}: u_{\gamma} \rightarrow u_{\gamma}^{\prime}$ in $\mathcal{B}$ such that the pair $\left(\operatorname{id}_{\Sigma}: \Sigma \rightarrow \Sigma, F\right)$ is an isomorphism between the $\mathcal{B}$-colored $G$-surfaces $(\Sigma, A, u)$ and $\left(\Sigma, A, u^{\prime}\right)$.

A 0 -system of tracks for $(\Sigma, A)$ is a family $\left\{\gamma_{a}\right\}_{a \in A}$ where $\gamma_{a}$ is a track of $a$. To produce such a system one just picks a track for each point of $A$ (this is always possible since every component of $\Sigma$ has a base point).

Given a 0 -system of tracks $\left\{\gamma_{a}\right\}_{a \in A}$ for $(\Sigma, A)$, any family $\left\{u_{a} \in \mathcal{B}_{\mu_{\gamma_{a}}}\right\}_{a \in A}$ determines a $\mathcal{B}$-coloring $u$ of $(\Sigma, A)$ as follows. Every track $\gamma$ of any $a \in A$ expands uniquely as $\gamma=\alpha \gamma_{a}$ with $\alpha \in \pi_{1}\left(\Sigma \backslash A, *_{a}\right)$. Set

$$
u_{\gamma}=\varphi_{\alpha^{-1}}\left(u_{a}\right) \in \mathcal{B}_{\alpha \mu_{\gamma_{a}} \alpha^{-1}}=\mathcal{B}_{\mu_{\gamma}} .
$$

In particular, $u_{\gamma_{a}}=\varphi_{1}\left(u_{a}\right)$ is canonically isomorphic to $u_{a}$. For $\gamma=\alpha \gamma_{a}$ as above and any $\beta \in \pi_{1}\left(\Sigma \backslash A, *_{a}\right)$, set

$$
u_{\beta, \gamma}=\left(\varphi_{2}\left(\beta^{-1}, \alpha^{-1}\right)_{u_{a}}\right)^{-1}: u_{\beta \gamma}=\varphi_{\alpha^{-1} \beta^{-1}}\left(u_{a}\right) \rightarrow \varphi_{\beta^{-1}} \varphi_{\alpha^{-1}}\left(u_{a}\right)=\varphi_{\beta^{-1}}\left(u_{\gamma}\right) .
$$


Lemma 6.1. The objects and isomorphisms above form a $\mathcal{B}$-coloring of $(\Sigma, A)$ which only depends (up to strong isomorphism) on the isomorphism classes of the objects $\left\{u_{a}\right\}_{a \in A}$. This construction yields all $\mathcal{B}$-colorings of $(\Sigma, A)$ up to strong isomorphism: any $\mathcal{B}$-coloring $U$ of $(\Sigma, A)$ is strongly isomorphic to the $\mathcal{B}$-coloring of $(\Sigma, A)$ determined by the family of objects $\left\{U_{\gamma_{a}}\right\}_{a \in A}$.

Lemma 6.1 is an analogue of Lemmas $5.1,5.2$ and is proven similarly.

6.4. Surfaces versus graphs. Colored $G$-surfaces and $G$-graphs are related in two ways: the boundary of any precolored $G$-graph is a colored $G$-surface and the cylinder over any colored $G$-surface is a colored $G$-graph. Here are more details. Consider a $\mathcal{B}$-precolored $G$-graph $((M, \Omega), u)$. We endow the pointed oriented surface $\partial M$ with the set of marked points $\partial \Omega=\partial M \cap \Omega$ where the distinguished tangent direction at any $a \in \partial \Omega$ is induced by the framing of $\Omega$ at $a$ and the sign of $a$ is + if the adjacent arc of $\Omega$ is directed inside $M$ and - otherwise. The given homotopy class of maps $\left(M \backslash \Omega,(\partial M) \bullet \cup \Omega_{\bullet}\right) \rightarrow(\mathbf{X}, \mathbf{x})$ restricts to a homotopy class of maps $(\partial M \backslash \partial \Omega,(\partial M) \bullet) \rightarrow(\mathbf{X}, \mathbf{x})$. This turns $(\partial M, \partial \Omega)$ into a marked $G$-surface. We define its $\mathcal{B}$-coloring $\partial u$. Each track $\gamma$ of a point $a \in \partial \Omega$ in $\partial M$ determines through the inclusion $\partial M \hookrightarrow M$ a track, again denoted by $\gamma$, of the arc of $\Omega$ adjacent to $a$. Set $(\partial u)_{\gamma}=u_{\gamma} \in \mathcal{B}_{\mu_{\gamma}}$ and $(\partial u)_{\beta, \gamma}=u_{\beta, \gamma}$ for any $\beta \in \pi_{1}\left(\partial M \backslash \partial \Omega, *_{a}\right)$ where $*_{a}$ is the only point of $(\partial M)$. lying in the same component of $\partial M$ as $a$. This gives a $\mathcal{B}$-colored $G$-surface $(\partial M, \partial \Omega, \partial u)$. It is clear that (strongly) isomorphic $\mathcal{B}$ precolorings of $(M, \Omega)$ yield in this way (strongly) isomorphic $\mathcal{B}$-colored $G$-surfaces.

The cylinder construction starts from an arbitrary $\mathcal{B}$-colored $G$-surface $(\Sigma, A, u)$. Let $I=[0,1]$ be the unit segment directed from 0 to 1 . Consider the 3 -manifold $C=$ $\Sigma \times I$ with the product orientation and pointed boundary where $(\partial C)_{\bullet}=\Sigma_{\bullet} \times\{0,1\}$. Then $\Omega=A \times I \subset C$ is a ribbon graph with arcs $\{a \times I\}_{a \in A}$ and no coupons. Each arc $a \times I$ carries a framing which is a lift of the given tangent direction at $a$. The arc $a \times I$ is directed towards $a \times\{0\}$ if the sign of $a$ is + and towards $a \times\{1\}$ otherwise. Since $C$ has no closed components, $\Omega_{\bullet}=\emptyset$. Composing the projection pr: $C \backslash \Omega \rightarrow \Sigma \backslash A$ with the given homotopy class of maps $\left(\Sigma \backslash A, \Sigma_{\bullet}\right) \rightarrow(\mathbf{X}, x)$, we obtain a homotopy class of maps $\left(C \backslash \Omega,(\partial C)_{\bullet}\right) \rightarrow(\mathbf{X}, \mathbf{x})$. This determines a $G$-graph $(C, \Omega)$. Every track $\gamma$ of the arc $a \times I$ of $\Omega$ projects to the track $\operatorname{pr}(\gamma)$ of $a$ in $\Sigma$, and we set $U_{\gamma}=u_{p r(\gamma)} \in \mathcal{B}_{\mu_{\gamma}}$. If $\gamma$ is composable with a detour $\beta$ in $C \backslash \Omega$, consider $\operatorname{pr}(\beta) \in \pi_{1}\left(\Sigma \backslash A, *_{a}\right)$ and set

$$
U_{\beta, \gamma}=u_{\operatorname{pr}(\beta), \operatorname{pr}(\gamma)}: U_{\beta \gamma}=u_{\operatorname{pr}(\beta \gamma)} \rightarrow \varphi_{\beta^{-1}}\left(u_{\operatorname{pr}(\gamma)}\right)=\varphi_{\beta^{-1}}\left(U_{\gamma}\right) .
$$

This defines a $\mathcal{B}$-precoloring $U$ of $(C, \Omega)$. Since the ribbon graph $\Omega$ has no coupons, the tuple $C_{\Sigma}=((C, \Omega), U)$ is a $\mathcal{B}$-colored $G$-graph. It is called the cylinder over $(\Sigma, A, u)$.

\section{The CATEgory $\operatorname{Cob}_{\mathcal{B}}^{G}$}

For each $G$-crossed category $\mathcal{B}$ over $\mathbb{k}$, we define a symmetric monoidal category $\operatorname{Cob}_{\mathcal{B}}^{G}$ whose objects are $\mathcal{B}$-colored $G$-surfaces and whose morphisms are certain equivalence classes of $\mathcal{B}$-colored $G$-graphs.

7.1. Objects and morphisms. The objects of $\operatorname{Cob}_{\mathcal{B}}^{G}$ are $\mathcal{B}$-colored $G$-surfaces (possibly, empty). For $\mathcal{B}$-colored $G$-surfaces $\Sigma_{0}, \Sigma_{1}$, a morphism $\Sigma_{0} \rightarrow \Sigma_{1}$ in $\operatorname{Cob}_{\mathcal{B}}^{G}$ is represented by a triple $(M, \Omega, h)$ consisting of a $\mathcal{B}$-colored $G$-graph $(M, \Omega)$ and an isomorphism of $\mathcal{B}$-colored $G$-surfaces

$$
h:\left(-\Sigma_{0}\right) \sqcup \Sigma_{1} \rightarrow \partial M=(\partial M, \partial \Omega) .
$$

We call such a triple $(M, \Omega, h)$ a $\mathcal{B}$-colored $G$-cobordism between $\Sigma_{0}$ and $\Sigma_{1}$. Note that the image of $\Sigma_{0}$ (respectively, $\Sigma_{1}$ ) under $h$ is a union of several connected 
components of the $\mathcal{B}$-colored $G$-surface $\partial M$. We denote this image by $\partial_{-} M$ (respectively, $\left.\partial_{+} M\right)$ so that $\partial M$ is the disjoint union of the $\mathcal{B}$-colored $G$-surfaces $\partial_{-} M$ and $\partial_{+} M$. The isomorphism $h$ restricts to isomorphisms of $\mathcal{B}$-colored $G$-surfaces $h_{-}:-\Sigma_{0} \rightarrow \partial_{-} M$ and $h_{+}: \Sigma_{1} \rightarrow \partial_{+} M$.

Two $\mathcal{B}$-colored $G$-cobordisms between $\Sigma_{0}$ and $\Sigma_{1}$ represent the same morphism $\Sigma_{0} \rightarrow \Sigma_{1}$ in $\operatorname{Cob}_{\mathcal{B}}^{G}$ if they are obtained from each other by a finite sequence of the following operations and their inverses:

(i) Isomorphism: one picks an isomorphism $f:(M, \Omega) \rightarrow\left(M^{\prime}, \Omega^{\prime}\right)$ of $\mathcal{B}$-colored $G$-graphs and replaces $(M, \Omega, h)$ with

$$
\left(M^{\prime}, \Omega^{\prime}, f h:\left(-\Sigma_{0}\right) \sqcup \Sigma_{1} \rightarrow\left(\partial M^{\prime}, \partial \Omega^{\prime}\right)\right) .
$$

(ii) Stabilization: one keeps $M, h$ and stabilizes $\Omega$ at an arc as in Section 4.6.

(iii) Conjugation: one picks a closed component $C$ of $M$ meeting $\Omega$ and conjugates $(C, \Omega \cap C)$ by an element of the group $G$ keeping the rest of $M, \Omega$ and the isomorphism $h$.

7.2. Composition of morphisms. Let $\Sigma_{0}, \Sigma_{1}, \Sigma_{2}$ be $\mathcal{B}$-colored $G$-surfaces and let $\chi_{1}: \Sigma_{0} \rightarrow \Sigma_{1}$ and $\chi_{2}: \Sigma_{1} \rightarrow \Sigma_{2}$ be morphisms in $\operatorname{Cob}_{\mathcal{B}}^{G}$ represented respectively by $\mathcal{B}$-colored $G$-cobordisms $\left(M_{1}, \Omega_{1}, h_{1}\right)$ and $\left(M_{2}, \Omega_{2}, h_{2}\right)$. The morphism $\chi_{2} \circ \chi_{1}$ is represented by the $\mathcal{B}$-colored $G$-cobordism between $\Sigma_{0}$ and $\Sigma_{2}$ defined as follows. First, gluing $M_{1}$ and $M_{2}$ along the diffeomorphism

$$
\left(h_{2}\right)_{-} \circ\left(h_{1}\right)_{+}^{-1}: \partial_{+}\left(M_{1}\right) \rightarrow \partial_{-}\left(M_{2}\right)
$$

we obtain a 3 -manifold $M$. The orientations of $M_{1}$ and $M_{2}$ determine an orientation of $M$ so that both natural embeddings $j_{1}: M_{1} \hookrightarrow M$ and $j_{2}: M_{2} \hookrightarrow M$ are orientation preserving. Clearly, $\partial M=\partial_{-} M \sqcup \partial_{+} M$ where $\partial_{-} M=j_{1}\left(\partial_{-}\left(M_{1}\right)\right)$ and $\partial_{+} M=j_{2}\left(\partial_{+}\left(M_{2}\right)\right)$. We endow $\partial M$ with the set of base points

$$
(\partial M)_{\bullet}=j_{1}\left(\left(\partial_{-}\left(M_{1}\right)\right)_{\bullet}\right) \cup j_{2}\left(\left(\partial_{+}\left(M_{2}\right)\right) \bullet\right) .
$$

Thus, $M$ is a compact oriented 3-manifold with pointed boundary. Note that the diffeomorphisms $\left(h_{1}\right)_{+}: \Sigma_{1} \rightarrow \partial_{+}\left(M_{1}\right)$ and $\left(h_{2}\right)_{-}: \Sigma_{1} \rightarrow \partial_{-}\left(M_{2}\right)$ induce an embedding $h: \Sigma_{1} \hookrightarrow M$ such that

$$
h\left(\Sigma_{1}\right)=j_{1}\left(\partial_{+}\left(M_{1}\right)\right)=j_{2}\left(\partial_{-}\left(M_{2}\right)\right) \subset \operatorname{Int}(M) .
$$

Since the map (3) is an isomorphism of $G$-surfaces, the endpoints of the arcs of $\Omega_{1}, \Omega_{2}$ in $\partial_{+}\left(M_{1}\right), \partial_{-}\left(M_{2}\right)$ match under the gluing and so do the orientations and the framings of the adjacent arcs of $\Omega_{1}, \Omega_{2}$. At each of these endpoints we insert in the union $j_{1}\left(\Omega_{1}\right) \cup j_{2}\left(\Omega_{2}\right)$ a small coupon with one input and one output as in Section 4.6. The bottom base of this coupon is its side lying in $j_{1}\left(M_{1}\right)$. The resulting set $\Omega \subset M$ contains $j_{1}\left(\Omega_{1}\right) \cup j_{2}\left(\Omega_{2}\right)$ and expands as a union of a finite number of arcs and coupons. The coupons of $\Omega$ are the images of the coupons of $\Omega_{1}, \Omega_{2}$ under $j_{1}, j_{2}$ and the coupons added above at the points of the set $h\left(\left(\Sigma_{1}\right)_{\bullet}\right)$. The arcs of $\Omega$ are the images of the arcs of $\Omega_{1}, \Omega_{2}$ under $j_{1}, j_{2}$ which are slightly shortened near $h\left(\left(\Sigma_{1}\right)_{\bullet}\right)$. The framings of $\Omega_{1}, \Omega_{2}$ extend to a framing of $\Omega$ in the obvious way. Clearly, $\Omega$ is a ribbon graph in $M$.

Each closed connected component $C$ of $M$ either lies in $j_{i}\left(M_{i}\right)$ for $i \in\{1,2\}$ or meets the surface $h\left(\Sigma_{1}\right)$ along several components. For $C$ meeting both $h\left(\Sigma_{1}\right)$ and $\Omega$, pick any point $p_{C} \in C \cap h\left(\left(\Sigma_{1}\right) \bullet\right)$. We endow $\Omega$ with the set of base points

$$
\Omega_{\bullet}=j_{1}\left(\left(\Omega_{1}\right) \bullet\right) \cup j_{2}\left(\left(\Omega_{2}\right)_{\bullet}\right) \cup\left\{p_{C}\right\}_{C}
$$

where $C$ runs over closed components of $M$ meeting both $h\left(\Sigma_{1}\right)$ and $\Omega$. This turns $\Omega$ into a pointed ribbon graph in $M$. Note the inclusion

$$
(\partial M) \bullet \cup \Omega_{\bullet} \subset j_{1}\left(\left(\partial M_{1}\right) \bullet \cup\left(\Omega_{1}\right)_{\bullet}\right) \cup j_{2}\left(\left(\partial M_{2}\right) \bullet \cup\left(\Omega_{2}\right)_{\bullet}\right) .
$$


Since the map (3) is an isomorphism of $G$-surfaces, we can pick representatives in the given homotopy classes of maps

$$
\left\{\left(M_{i} \backslash \Omega_{i},\left(\partial M_{i}\right) \bullet \cup\left(\Omega_{i}\right) \bullet\right) \rightarrow(\mathbf{X}, x)\right\}_{i=1,2}
$$

which match under (3). These representatives determine a map

$$
\left(M \backslash \Omega, \quad j_{1}\left(\left(\partial M_{1}\right) \bullet \cup\left(\Omega_{1}\right)_{\bullet}\right) \cup j_{2}\left(\left(\partial M_{2}\right) \bullet \cup\left(\Omega_{2}\right)_{\bullet}\right)\right) \rightarrow(\mathbf{X}, x) .
$$

In view of the inclusion (4), the latter map determines a homotopy class of maps

$$
\left(M \backslash \Omega,(\partial M) \bullet \cup \Omega_{\bullet}\right) \rightarrow(\mathbf{X}, x) .
$$

Since $\mathbf{X}=K(G, 1)$, an elementary obstruction theory shows that this homotopy class does not depend on the choice of representatives of the homotopy classes (5). In this way, $(M, \Omega)$ becomes a $G$-graph.

Let $\left(u^{i}, v^{i}\right)$ be the $\mathcal{B}$-coloring of $\left(M_{i}, \Omega_{i}\right)$ for $i=1,2$. We use the method of Section 5.3 to derive from these colorings a $\mathcal{B}$-coloring $(u, v)$ of $(M, \Omega)$. For $i=1,2$, pick a 1-system of tracks $\left\{\gamma_{e}^{i}\right\}_{e}$ for $\left(M_{i}, \Omega_{i}\right)$ where $e$ runs over the arcs of $\Omega_{i}$. Consider any track $\gamma$ in $M \backslash \Omega$ of an arc of $\Omega$. This arc lies in $j_{i}(e)$ for some $i=1,2$ and some arc $e$ of $\Omega_{i}$. Then $\gamma$ expands uniquely as the product $\gamma=\alpha j_{i}\left(\gamma_{e}^{i}\right)$ where $\alpha$ is a homotopy class of paths in $M \backslash \Omega$ from $\gamma(0) \in(\partial M) \bullet \cup \Omega$ • to

$$
j_{i}\left(\gamma_{e}^{i}(0)\right) \in j_{i}\left(\left(\partial M_{i}\right) \bullet \cup\left(\Omega_{i}\right)_{\bullet}\right) .
$$

The map (6) carries $\alpha$ into a homotopy class of loops in $(\mathbf{X}, \mathbf{x})$ representing an element of $G=\pi_{1}(\mathbf{X}, \mathbf{x})$ also denoted by $\alpha$. Set

$$
u_{\gamma}=\varphi_{\alpha^{-1}}\left(u_{\gamma_{e}^{i}}^{i}\right) \in \mathcal{B}_{\alpha \mu_{\gamma_{e}^{i} \alpha^{-1}}}=\mathcal{B}_{\mu_{\gamma}}
$$

For any detour $\beta$ in $M \backslash \Omega$ composable with $\gamma$, set

$u_{\beta, \gamma}=\left(\varphi_{2}\left(\beta^{-1}, \alpha^{-1}\right)_{u_{\gamma_{e}^{i}}^{i}}\right)^{-1}: u_{\beta \gamma}=\varphi_{\alpha^{-1} \beta^{-1}}\left(u_{\gamma_{e}^{i}}^{i}\right) \rightarrow \varphi_{\beta^{-1}} \varphi_{\alpha^{-1}}\left(u_{\gamma_{e}^{i}}^{i}\right)=\varphi_{\beta^{-1}}\left(u_{\gamma}\right)$.

This defines a $\mathcal{B}$-precoloring $u$ of $(M, \Omega)$. We now define a coupon-coloring $v$ of $((M, \Omega), u)$. For $i=1,2$ pick a 2 -system of tracks $\left\{\gamma_{c}^{i}\right\}_{c}$ of $\Omega_{i}$ where $c$ runs over the coupons of $\Omega_{i}$. Also pick a 0 -system of tracks $\left\{\gamma_{a}\right\}_{a}$ for $\Sigma_{1}$ where $a$ runs over the marked points of $\Sigma_{1}$. For each track $\gamma$ of a coupon of $\Omega$, we define a morphism $v_{\gamma}: u_{\gamma} \rightarrow u^{\gamma}$ in $\mathcal{B}$ as follows. Assume first that the coupon in question is $j_{i}(c)$ where $i=1,2$ and $c$ is a coupon of $\Omega_{i}$. Then $\gamma$ expands uniquely as the product $\gamma=\alpha j_{i}\left(\gamma_{c}^{i}\right)$ where $\alpha$ is a homotopy class of paths in $M \backslash \Omega$ from $\gamma(0) \in(\partial M) \bullet \cup \Omega$. to

$$
j_{i}\left(\gamma_{c}^{i}(0)\right) \in j_{i}\left(\left(\partial M_{i}\right) \bullet \cup\left(\Omega_{i}\right)_{\bullet}\right) .
$$

As above, we use the same letter $\alpha$ to denote the element of $G$ represented by the image of $\alpha$ in $\mathbf{X}$. We use the notation of Section 4.5 for the data associated with the coupon $c$ and its track $\gamma_{c}^{i}$ in $M_{i} \backslash \Omega_{i}$. Consider the morphism

$$
v_{\gamma_{c}^{i}}^{i}:\left(u^{i}\right)_{\gamma_{c}^{i}} \rightarrow\left(u^{i}\right)^{\gamma_{c}^{i}} \quad \text { where } \quad\left(u^{i}\right)_{\gamma_{c}^{i}}=\bigotimes_{k=1}^{m}\left(u_{\rho_{k}}^{i}\right)^{\varepsilon_{k}} \quad \text { and } \quad\left(u^{i}\right)^{\gamma_{c}^{i}}=\bigotimes_{\ell=1}^{n}\left(u_{\rho^{\ell}}^{i}\right)^{\varepsilon^{\ell}} .
$$

(Recall that $X^{+}=X$ and $X^{-}=X^{*}$ for any object $X \in \mathcal{B}$.) The composition of the track $\gamma$ of the coupon $j_{i}(c) \subset \Omega$ with a path in the parallel coupon $\widetilde{j_{i}(c)} \subset \widetilde{\Omega}$ leading to the $k$-th input is the product track $\alpha j_{i}\left(\rho_{k}\right)$. Hence

$$
u_{\gamma}=\bigotimes_{k=1}^{m} u_{\alpha j_{i}\left(\rho_{k}\right)}^{\varepsilon_{k}}=\bigotimes_{k=1}^{m}\left(\varphi_{\alpha^{-1}}\left(u_{\rho_{k}}^{i}\right)\right)^{\varepsilon_{k}}
$$

Similarly,

$$
u^{\gamma}=\bigotimes_{\ell=1}^{n} u_{\alpha j_{i}\left(\rho^{\ell}\right)}^{\varepsilon^{\ell}}=\bigotimes_{\ell=1}^{n}\left(\varphi_{\alpha^{-1}}\left(u_{\rho^{\ell}}^{i}\right)\right)^{\varepsilon^{\ell}}
$$


Define the morphism $v_{\gamma}: u_{\gamma} \rightarrow u^{\gamma}$ as the following composition:

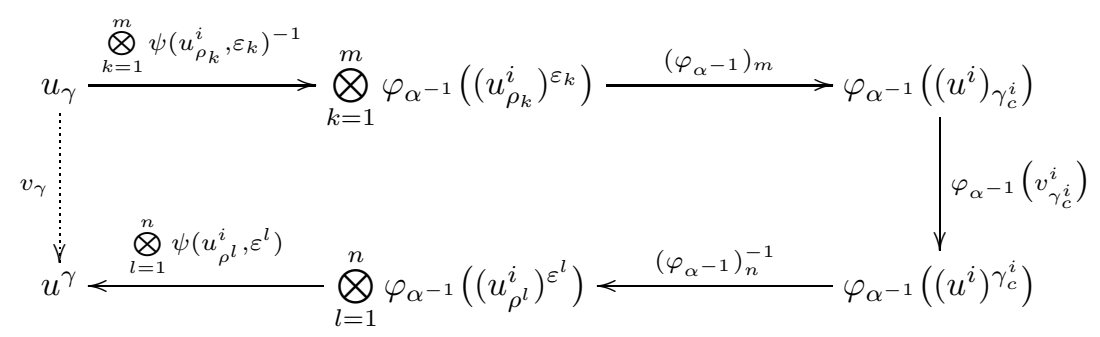

where, for an object $X \in \mathcal{B}$, we set $\psi(X,+)=\operatorname{id}_{\varphi_{\alpha-1}(X)}$ and $\psi(X,-)=\varphi_{\alpha^{-1}}^{1}(X)$. Next, consider a track $\gamma$ of the coupon $c_{a}$ of $\Omega$ at a marked point $a$ of $\Sigma_{1}$. We expand (uniquely) $\gamma=\alpha h\left(\gamma_{a}\right)$ where $h: \Sigma_{1} \hookrightarrow \operatorname{Int}(M)$ is the embedding above and $\alpha$ is a homotopy class of paths in $M \backslash \Omega$ from $\gamma(0) \in(\partial M) \bullet \cup \Omega$ • to

$$
h\left(\gamma_{a}(0)\right) \in h\left(\left(\Sigma_{1}\right)_{\bullet}\right)=j_{1}\left(\partial_{+}\left(M_{1}\right)_{\bullet}\right)=j_{2}\left(\partial_{-}\left(M_{2}\right)_{\bullet}\right) \subset h\left(\Sigma_{1}\right) .
$$

For $i=1,2$ the point $h_{i}(a) \in \partial \Omega_{i}$ is an endpoint of a unique arc $e_{i}$ of $\Omega_{i}$. Then $h_{i}\left(\gamma_{a}\right)$ is a track of $e_{i}$ in $M_{i} \backslash \Omega_{i}$, and $h_{i}\left(\gamma_{a}\right)=\beta_{i} \gamma_{e_{i}}^{i}$ for a unique detour $\beta_{i}$ in $M_{i} \backslash \Omega_{i}$. The isomorphisms of $\mathcal{B}$-colored $G$-surfaces (3) induces an isomorphism

For $i=1,2$, set

$$
\Phi: u_{\beta_{1} \gamma_{e_{1}}^{1}}^{1}=u_{h_{1}\left(\gamma_{a}\right)}^{1} \rightarrow u_{h_{2}\left(\gamma_{a}\right)}^{2}=u_{\beta_{2} \gamma_{e_{2}}^{2}}^{2} .
$$

$$
\Psi_{i}=\varphi_{2}\left(\alpha^{-1}, \beta_{i}^{-1}\right)_{u_{\gamma_{e_{i}}^{i}}^{i}} \circ \varphi_{\alpha^{-1}}\left(u_{\beta_{i}, \gamma_{e_{i}}^{i}}^{i}\right): \varphi_{\alpha^{-1}}\left(u_{\beta_{i} \gamma_{e_{i}}^{i}}^{i}\right) \rightarrow \varphi_{\beta_{i}^{-1} \alpha^{-1}}\left(u_{\gamma_{e_{i}}^{i}}^{i}\right) .
$$

Let $\varepsilon=\varepsilon_{a}$ be the sign carried by $a$. Clearly, the composition of the track $\gamma$ with a path in the parallel coupon $\widetilde{c_{a}}$ leading to its unique input is the track $\alpha j_{1}\left(\beta_{1} \gamma_{e_{1}}^{1}\right)$ of the $\operatorname{arc} j_{1}\left(e_{1}\right)$ of $\Omega$. Then

$$
u_{\gamma}=\left(\varphi_{\left(\alpha \beta_{1}\right)^{-1}}\left(u_{\gamma_{e_{1}}^{1}}^{1}\right)\right)^{\varepsilon}=\left(\varphi_{\beta_{1}^{-1} \alpha^{-1}}\left(u_{\gamma_{e_{1}}^{1}}^{1}\right)\right)^{\varepsilon}
$$

Considering the output of $\widetilde{c_{a}}$, we similarly obtain that

$$
u^{\gamma}=\left(\varphi_{\left(\alpha \beta_{2}\right)^{-1}}\left(u_{\gamma_{e_{2}}^{2}}^{2}\right)\right)^{\varepsilon}=\left(\varphi_{\beta_{2}^{-1} \alpha^{-1}}\left(u_{\gamma_{e_{2}}^{2}}^{2}\right)\right)^{\varepsilon} .
$$

Define the morphism $v_{\gamma}: u_{\gamma} \rightarrow u^{\gamma}$ as

$$
v_{\gamma}= \begin{cases}\Psi_{2} \circ \varphi_{\alpha^{-1}}(\Phi) \circ\left(\Psi_{1}\right)^{-1} & \text { if } \varepsilon=+, \\ \left(\Psi_{1} \circ \varphi_{\alpha^{-1}}\left(\Phi^{-1}\right) \circ\left(\Psi_{2}\right)^{-1}\right)^{*} & \text { if } \varepsilon=-.\end{cases}
$$

This defines a coupon-coloring $v$ of $((M, \Omega), u)$ and a $\mathcal{B}$-coloring $(u, v)$ of $(M, \Omega)$.

It follows from the definition of $u$ that the embedding $j_{1}: M_{1} \hookrightarrow M$ induces an isomorphism of $\mathcal{B}$-colored $G$-surfaces $\partial_{-}\left(M_{1}\right) \rightarrow \partial_{-} M$. Composing with the isomorphism $\left(h_{1}\right)_{-}:-\Sigma_{0} \rightarrow \partial_{-}\left(M_{1}\right)$, we obtain an isomorphism $-\Sigma_{0} \rightarrow \partial_{-} M$. Similarly, $j_{2}$ and $\left(h_{2}\right)_{+}$induce an isomorphism $\Sigma_{2} \rightarrow \partial_{+} M$. This yields an isomorphism of $\mathcal{B}$-colored $G$-surfaces

$$
\left(-\Sigma_{0}\right) \sqcup \Sigma_{2} \rightarrow \partial M=(\partial M, \partial \Omega, \partial u) .
$$

The $\mathcal{B}$-colored $G$-graph $((M, \Omega), u, v)$ endowed with this isomorphism is a $\mathcal{B}$-colored $G$-cobordism between $\Sigma_{0}$ and $\Sigma_{2}$ which represents the composition $\chi_{2} \circ \chi_{1}$ in $\operatorname{Cob}_{\mathcal{B}}^{G}$. This composition is well-defined: it is independent of the choice of the points $\left\{p_{C}\right\}_{C}$ and the tracks above. In particular, if $p_{C} \in C \cap h\left(\left(\Sigma_{1}\right) \bullet\right)$ is replaced with $p_{C}^{\prime} \in$ $C \cap h\left(\left(\Sigma_{1}\right)_{\bullet}\right)$, then there is an isotopy $\left\{f_{t}: M \rightarrow M\right\}_{t \in I}$ such that $f_{0}=\operatorname{id}_{M}$, $\left.f_{t}\right|_{\Omega}=\operatorname{id}_{\Omega}$ and $\left.f_{t}\right|_{M \backslash C}=\operatorname{id}_{M \backslash C}$ for all $t \in I$, and $f_{1}\left(p_{C}\right)=p_{C}^{\prime}$. The $\mathcal{B}$-colored $G$ cobordisms derived from $p_{C}$ and $p_{C}^{\prime}$ are related via the diffeomorphism $f_{1}$ and the conjugation by the element of $G$ represented by the path $t \in I \mapsto f_{t}\left(p_{C}\right) \in C \backslash \Omega$. Also, this composition of morphisms in $\operatorname{Cob}_{\mathcal{B}}^{G}$ is associative. 
7.3. Further structures in $\operatorname{Cob}_{\mathcal{B}}^{G}$. All objects of $\operatorname{Cob}_{\mathcal{B}}^{G}$ have identity endomorphisms in $\operatorname{Cob}_{\mathcal{B}}^{G}$. Namely, for a $\mathcal{B}$-colored $G$-surface $\Sigma$, the cylinder over $\Sigma$ (see Section 6.4) endowed with the standard identification of its boundary with $(-\Sigma) \sqcup \Sigma$ is a $\mathcal{B}$-colored $G$-cobordism representing $\operatorname{id}_{\Sigma}$ in $\operatorname{Cob}_{\mathcal{B}}^{G}$. These identity endomorphisms are identities for the composition in $\operatorname{Cob}_{\mathcal{B}}^{G}$. $\operatorname{So}, \operatorname{Cob}_{\mathcal{B}}^{G}$ is a category.

A more general construction derives from any isomorphism $f: \Sigma \rightarrow \Sigma^{\prime}$ between $\mathcal{B}$-colored $G$-surfaces a morphism $\Sigma \rightarrow \Sigma^{\prime}$ in $\operatorname{Cob}_{\mathcal{B}}^{G}$ called the cylinder of $f$ and denoted cyl $(f)$. This morphism is represented by the $\mathcal{B}$-colored $G$-cobordism between $\Sigma$ and $\Sigma^{\prime}$ which is formed by the cylinder $C_{\Sigma^{\prime}}$ over $\Sigma^{\prime}$ together with the isomorphism of $\mathcal{B}$-colored $G$-surfaces $(-\Sigma) \sqcup \Sigma^{\prime} \rightarrow \partial C_{\Sigma^{\prime}}$ carrying any $x \in \Sigma$ to $(f(x), 0)$ and any $x^{\prime} \in \Sigma^{\prime}$ to $\left(x^{\prime}, 1\right)$. It is easy to check that if $f: \Sigma \rightarrow \Sigma^{\prime}$ and $g: \Sigma^{\prime} \rightarrow \Sigma^{\prime \prime}$ are composable isomorphisms between $\mathcal{B}$-colored $G$-surfaces, then $\operatorname{cyl}(g f)=\operatorname{cyl}(g) \circ \operatorname{cyl}(f)$. Consequently, the cylinders are isomorphisms in $\mathrm{Cob}_{\mathcal{B}}^{G}$.

The disjoint union of $\mathcal{B}$-colored $G$-surfaces and $G$-cobordisms turns $\operatorname{Cob}_{\mathcal{B}}^{G}$ into a symmetric monoidal category. Its unit object is the empty set $\emptyset$ viewed as a $\mathcal{B}$-colored $G$-surface. The associativity and unitality constraints in $\operatorname{Cob}_{\mathcal{B}}^{G}$ are the cylinders of the tautological isomorphisms

$$
\left(\Sigma \sqcup \Sigma^{\prime}\right) \sqcup \Sigma^{\prime \prime} \simeq \Sigma \sqcup\left(\Sigma^{\prime} \sqcup \Sigma^{\prime \prime}\right) \text { and } \emptyset \sqcup \Sigma \simeq \Sigma \simeq \Sigma \sqcup \emptyset,
$$

where $\Sigma, \Sigma^{\prime}, \Sigma^{\prime \prime}$ run over all $\mathcal{B}$-colored $G$-surfaces. The symmetry in $\operatorname{Cob}_{\mathcal{B}}^{G}$ is determined by the cylinders of the obvious permutation isomorphisms

$$
\Sigma \otimes \Sigma^{\prime}=\Sigma \sqcup \Sigma^{\prime} \simeq \Sigma^{\prime} \sqcup \Sigma=\Sigma^{\prime} \otimes \Sigma .
$$

The category $\operatorname{Cob}_{\mathcal{B}}^{G}$ has a canonical left duality $\left\{\left(-\Sigma, \mathrm{ev}_{\Sigma}\right)\right\}_{\Sigma}$, where $\Sigma$ runs over all $\mathcal{B}$-colored $G$-surfaces. Here $-\Sigma$ is the opposite $\mathcal{B}$-colored $G$-surface (see Section 6.2) and the morphism $\mathrm{ev}_{\Sigma}:(-\Sigma) \otimes \Sigma \rightarrow \emptyset$ in $\operatorname{Cob}_{\mathcal{B}}^{G}$ is represented by the $\mathcal{B}$-colored $G$-cobordism formed by the cylinder $C_{-\Sigma}$ over $-\Sigma$ together with the isomorphism of $\mathcal{B}$-colored $G$-surfaces

$$
-((-\Sigma) \otimes \Sigma) \sqcup \emptyset=\Sigma \sqcup(-\Sigma) \simeq(\Sigma \times\{0\}) \sqcup(-\Sigma \times\{1\})=\partial C_{-\Sigma} .
$$

This left duality turns the symmetric monoidal category $\operatorname{Cob}_{\mathcal{B}}^{G}$ into a ribbon category with trivial twist (see [TV5, Lemma 3.5]). In particular, $\operatorname{Cob}_{\mathcal{B}}^{G}$ is spherical.

The category $\mathrm{Cob}^{G}$ defined in Section 3.1 is a symmetric monoidal subcategory of $\mathrm{Cob}_{\mathcal{B}}^{G}$. Indeed, any $G$-surface in the sense of Section 3.1 is a $\mathcal{B}$-colored $G$ surface with an empty set of marked points (so that the notion of a $\mathcal{B}$-coloring for this surface is void). Also, any $G$-cobordism between $G$-surfaces (in the sense of Section 3.1) is a $\mathcal{B}$-colored $G$-cobordism with an empty ribbon graph (so that the notion of a $\mathcal{B}$-coloring for this cobordism is void). This defines an embedding of categories $\mathrm{Cob}^{G} \hookrightarrow \mathrm{Cob}_{\mathcal{B}}^{G}$ which is symmetric strict monoidal.

\section{Graph HQFTs And THE MAIN THEOREM}

8.1. Graph HQFTs. Let $\mathcal{B}$ be a $G$-crossed category over $\mathbb{k}$. A graph HQFT over $\mathcal{B}$ with target $\mathbf{X}=K(G, 1)$ is a symmetric strong monoidal functor $Z$ : $\operatorname{Cob}_{\mathcal{B}}^{G} \rightarrow \operatorname{Mod}_{\mathbb{k}}$ where $\operatorname{Cob}_{\mathcal{B}}^{G}$ is the symmetric monoidal category defined in Section 7 and $\operatorname{Mod}_{\mathbb{k}}$ is the symmetric monoidal category of $\mathbb{k}$-modules and $\mathbb{k}$-linear homomorphisms. Such a functor includes $\mathbb{k}$-linear isomorphisms

$$
Z_{0}: \mathbb{k} \stackrel{\sim}{\longrightarrow} Z(\emptyset) \text { and } Z_{2}\left(\Sigma, \Sigma^{\prime}\right): Z(\Sigma) \otimes_{\mathbb{k}} Z\left(\Sigma^{\prime}\right) \stackrel{\sim}{\longrightarrow} Z\left(\Sigma \sqcup \Sigma^{\prime}\right)
$$

for any $\mathcal{B}$-colored $G$-surfaces $\Sigma, \Sigma^{\prime}$.

Recall that a morphism $\emptyset \rightarrow \emptyset$ in $\operatorname{Cob}_{\mathcal{B}}^{G}$ is represented by a triple $\left(M, \Omega, \operatorname{id}_{\emptyset}\right)$, where $M$ is a closed oriented 3 -manifold and $\Omega$ is a $\mathcal{B}$-colored $G$-graph in $M$. Applying $Z$ to this morphism we get a $\mathbb{k}$-linear homomorphism $Z(\emptyset) \rightarrow Z(\emptyset)$. Since $Z(\emptyset) \simeq \mathbb{k}$, the latter homomorphism is multiplication by an element of $\mathbb{k}$. 
This element is denoted $Z(M, \Omega)$ and is an isomorphism invariant of $\mathcal{B}$-colored $G$-graphs in closed oriented 3-manifolds.

As in topological quantum field theory (TQFT), for any $\mathcal{B}$-colored $G$-surface $\Sigma$, the $\mathbb{k}$-module $Z(\Sigma)$ is projective of finite type (see, for example, TV5]). Clearly, the isomorphism class of this module is preserved under isotopy of the set of marked points in $\Sigma$. Also, each connected component $\Gamma$ of $\Sigma$ may be treated as a $\mathcal{B}$-colored $G$-surface whose marked points are the marked points of $\Sigma$ belonging to $\Gamma$. The strong monoidality and the symmetry of $Z$ yield a $\mathbb{k}$-linear isomorphism

$$
Z(\Sigma) \simeq \bigotimes_{\Gamma} Z(\Gamma)
$$

where $\Gamma$ runs over connected components of $\Sigma$ and $\otimes$ is the unordered tensor product of $\mathbb{k}$-modules.

By Section 7.3, $\mathcal{B}$-colored $G$-surfaces and the cylinders of their isomorphisms form a (non-full) symmetric monoidal subcategory of $\mathrm{Cob}_{\mathcal{B}}^{G}$. It is denoted Homeo ${ }_{\mathcal{B}}^{G}$. Restricting a graph HQFT $Z$ to $\operatorname{Homeo}_{\mathcal{B}}^{G}$, we obtain a symmetric monoidal functor Homeo $_{\mathcal{B}}^{G} \rightarrow \operatorname{Mod}_{\mathbb{k}}$. In particular, $Z$ induces a $\mathbb{k}$-linear representation of the group of isotopy classes of automorphisms of $\Sigma$.

A graph HQFT $Z$ over $\mathcal{B}$ is non-degenerate if for any $\mathcal{B}$-colored $G$-surface $\Sigma$, the $\mathbb{k}$-module $Z(\Sigma)$ is spanned by the images of the homomorphisms $Z(\emptyset) \rightarrow Z(\Sigma)$ induced by the morphisms $\emptyset \rightarrow \Sigma$ in $\operatorname{Cob}_{\mathcal{B}}^{G}$. Formula (7) and the strong monoidality of $Z$ imply that if this condition holds for all connected $\mathcal{B}$-colored $G$-surfaces, then it holds for all disconnected $\mathcal{B}$-colored $G$-surfaces as well.

An isomorphism between graph HQFTs $Z, Z^{\prime}: \operatorname{Cob}_{\mathcal{B}}^{G} \rightarrow \operatorname{Mod}_{\mathbb{k}}$ is a monoidal natural isomorphism $Z \rightarrow Z^{\prime}$, i.e., a family of $\mathbb{k}$-linear isomorphisms $\left\{\rho_{\Sigma}: Z(\Sigma) \rightarrow\right.$ $\left.Z^{\prime}(\Sigma)\right\}_{\Sigma}$ where $\Sigma$ runs over all $\mathcal{B}$-colored $G$-surfaces. These isomorphisms should commute with the action of $\mathcal{B}$-colored $G$-cobordisms, be multiplicative under disjoint unions of $\mathcal{B}$-colored $G$-surfaces, and satisfy $\rho_{\emptyset}=Z_{0}^{\prime} Z_{0}^{-1}$. It is clear that if $Z$ and $Z^{\prime}$ are isomorphic, then $Z(M, \Omega)=Z^{\prime}(M, \Omega)$ for any $\mathcal{B}$-colored $G$-graph $\Omega$ in a closed oriented 3-manifold $M$.

Recall from Section 3.2 that a 3 -dimensional HQFT (over $\mathbb{k}$ ) is a symmetric strong monoidal functor $\mathrm{Cob}^{G} \rightarrow \operatorname{Mod}_{\mathbb{k}}$. Let $J$ be the embedding $\operatorname{Cob}^{G} \hookrightarrow \mathrm{Cob}_{\mathcal{B}}^{G}$ defined in Section 7.3. A graph extension along $\mathcal{B}$ of a 3-dimensional HQFT $Z: \operatorname{Cob}^{G} \rightarrow \operatorname{Mod}_{\mathbb{k}}$ is a graph $\mathrm{HQFT} \widetilde{Z}: \operatorname{Cob}_{\mathcal{B}}^{G} \rightarrow \operatorname{Mod}_{\mathbb{k}}$ such that $\widetilde{Z} \circ J=Z$ as symmetric monoidal functors. Clearly, two 3-dimensional HQFTs having isomorphic graph extensions are themselves isomorphic.

8.2. The surgery graph HQFT. Let $\mathcal{B}$ be an anomaly free $G$-modular category over $\mathbb{k}$ (see Section 2.7). In TV4 we derive from $\mathcal{B}$ an $\operatorname{HQFT} \tau_{\mathcal{B}}: \mathrm{Cob}^{G} \rightarrow \operatorname{Mod}_{\mathbb{k}}$ called the surgery HQFT. The methods of [Tu2, [TV4] yield a non-degenerate graph HQFT $\operatorname{Cob}_{\mathcal{B}}^{G} \rightarrow \operatorname{Mod}_{\mathbb{k}}$ extending $\tau_{\mathcal{B}}$ and called the surgery graph HQFT. It is also denoted by $\tau_{\mathcal{B}}$. We state here two formulas computing $\tau_{\mathcal{B}}$ for closed surfaces and for graphs in closed 3-manifolds.

For a connected $\mathcal{B}$-colored $G$-surface $\Sigma$ of genus $g \geq 0$, the $\mathbb{k}$-module $\tau_{\mathcal{B}}(\Sigma)$ is computed (up to isomorphism) as follows. The surface $\Sigma$ carries a base point $*$, a finite set of marked points $A=\left\{a_{1}, \ldots, a_{m}\right\} \subset \Sigma \backslash\{*\}$, and a homotopy class of maps $(\Sigma \backslash A, *) \rightarrow(\mathbf{X}, \mathbf{x})$. Pick a track $\gamma_{i}$ of $a_{i}$ for $i=1, \ldots, m$ so that these $m$ tracks can be represented by paths in $\Sigma \backslash A$ meeting only in their starting point $*$. Recall from Section 6.1 the homotopy class $\mu_{i}=\mu_{\gamma_{i}} \in \pi_{1}(\Sigma \backslash A, *)$ of the loop encircling $a_{i}$. The group $\pi_{1}(\Sigma \backslash A, *)$ is generated by $\mu_{1}, \ldots, \mu_{m}$ and $2 g$ elements $\alpha_{1}, \beta_{1}, \ldots, \alpha_{g}, \beta_{g}$ subject to the only relation

$$
\left(\alpha_{1}^{-1} \beta_{1}^{-1} \alpha_{1} \beta_{1}\right) \cdots\left(\alpha_{g}^{-1} \beta_{g}^{-1} \alpha_{g} \beta_{g}\right)\left(\mu_{1}\right)^{\varepsilon_{1}} \cdots\left(\mu_{m}\right)^{\varepsilon_{m}}=1,
$$


where $\varepsilon_{i}= \pm 1$ is the sign of $a_{i}$. As usual, denote the element of $G$ represented by the image of $\mu_{i}$ in $\mathbf{X}$ by the same symbol $\mu_{i}$, and similarly for $\alpha_{j}, \beta_{j}$. Let $u_{i} \in \mathcal{B}_{\mu_{i}}$ be the color of $\gamma_{i}$. Set

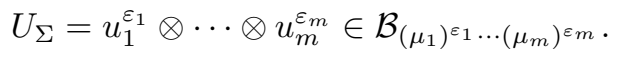

(If $m=0$, then $U_{\Sigma}=\mathbb{1} \in \mathcal{B}_{1}$.) Let $\mathcal{J}=\amalg_{\alpha \in G} \mathcal{J}_{\alpha}$ be a representative set of simple objects of $\mathcal{B}$. Then there is an $\mathbb{k}$-linear isomorphism

$$
\text { (8) } \tau_{\mathcal{B}}(\Sigma) \simeq \underset{J_{1} \in \mathcal{J}_{\beta_{1}}, \ldots, J_{g} \in \mathcal{J}_{\beta g}}{\operatorname{Hom}_{\mathcal{B}}}\left(\mathbb{1}_{\mathcal{B}},\left(\varphi_{\alpha_{1}}\left(J_{1}\right)^{*} \otimes J_{1}\right) \otimes \cdots \otimes\left(\varphi_{\alpha_{g}}\left(J_{g}\right)^{*} \otimes J_{g}\right) \otimes U_{\Sigma}\right) .
$$

We recall the definition of the scalar invariant $\tau_{\mathcal{B}}(M, \Omega) \in \mathbb{k}$ of a $\mathcal{B}$-colored $G$-graph $(M, \Omega)$ where $M$ is a closed connected oriented 3-manifold. The LickorishWallace theorem on surgery presentations of 3 -manifolds implies that there are a framed link $L=L_{1} \cup \cdots \cup L_{n}$ in $\mathbb{R}^{2} \times(0,1)$ and a ribbon graph $\Omega^{\prime}$ in $\left(\mathbb{R}^{2} \times(0,1)\right) \backslash L$ such that the surgery on $S^{3}=\mathbb{R}^{3} \cup\{\infty\}$ along $L$ turns the pair $\left(S^{3}, \Omega^{\prime}\right)$ into $(M, \Omega)$, at least up to an orientation-preserving homeomorphism. Set

$$
E=\left(\mathbb{R}^{2} \times(0,1)\right) \backslash\left(\Omega^{\prime} \cup L\right) \subset M \backslash \Omega
$$

and take any point $z \in E$ with big second coordinate. We can assume that $M_{\bullet}=$ $\Omega_{\bullet}=\{z\}$. Restricting the given homotopy class of maps $\left(M \backslash \Omega, M_{\bullet}\right) \rightarrow(\mathbf{X}, \mathbf{x})$ to $E$ we obtain a homotopy class of maps $(E, z) \rightarrow(\mathbf{X}, \mathbf{x})$. Orient the link $L$ arbitrarily. Stabilize each component $L_{i}$ of $L$ by inserting into it a small coupon $c_{i}$ as in Section 4.6. We choose the bottom base of $c_{i}$ so that its unique input is directed out of $c_{i}$. This turns $\Omega^{\prime} \cup L$ into a $G$-graph in $\mathbb{R}^{2} \times(0,1)$ denoted $\bar{\Omega}$. For each $i \in\{1, \ldots, n\}$, pick a track $\gamma(i)$ of $c_{i}$ and set $\mu_{i}=\mu_{\gamma(i)} \in \pi_{1}(E, z)$. Let $\operatorname{col}(L)$ be the set of all maps $\lambda:\{1, \ldots, n\} \rightarrow \mathcal{J}$ such that $\lambda(i) \in \mathcal{B}_{\mu_{i}}$ for all $i \in\{1, \ldots, n\}$. Any such $\lambda$ together with the $\mathcal{B}$-coloring $(u, v)$ of $\Omega$ determine a $\mathcal{B}$-coloring $(\bar{u}, \bar{v})$ of $\bar{\Omega}$ as follows. Pick a 1 -system of tracks $\left\{\gamma_{e}^{\prime}\right\}_{e}$ for $\Omega^{\prime}$. For each $i \in\{1, \ldots, n\}$, the composition of the track $\gamma(i)$ with a path in the parallel coupon $\widetilde{c_{i}}$ (obtained by slightly pushing $c_{i}$ along the framing) leading to its unique input is a track $\gamma_{i}$ of the single arc of $\bar{\Omega}$ contained in $L_{i}$. Note that $\mu_{\gamma_{i}}=\mu_{i}$. Then the family $\left\{\gamma_{e}^{\prime}\right\}_{e} \cup\left\{\gamma_{i}\right\}_{i}$ is a 1-system of tracks for $\bar{\Omega}$. By Lemma [5.1, the family of objects $\left\{u_{\gamma_{e}^{\prime}}\right\}_{e} \cup\{\lambda(i)\}_{i}$ determines a $\mathcal{B}$-precoloring $\bar{u}$ of $\bar{\Omega}$ such that $\bar{u}_{\gamma_{e}^{\prime}}=\varphi_{1}\left(u_{\gamma_{e}^{\prime}}\right)$ and $\bar{u}_{\gamma_{i}}=\varphi_{1}(\lambda(i))$. Pick a 2-system of tracks $\left\{\gamma_{c}^{\prime}\right\}_{c}$ for $\Omega^{\prime}$. Then the family $\left\{\gamma_{c}^{\prime}\right\}_{c} \cup\{\gamma(i)\}_{i}$ is a 2system of tracks for $\bar{\Omega}$. Consider the $\mathcal{B}$-coloring $\left(u^{1}, v^{1}\right)$ of $\Omega^{\prime}$ obtained from $(u, v)$ by conjugation by $1 \in G$ (see Section 4.6). It follows from the definition that for any coupon $c$ of $\Omega^{\prime},(\bar{u})_{\gamma_{c}^{\prime}}=\left(u^{1}\right)_{\gamma_{c}^{\prime}}$ and $(\bar{u})^{\gamma_{c}^{\prime}}=\left(u^{1}\right)^{\gamma_{c}^{\prime}}$. For each $i \in\{1, \ldots, n\}$, the composition of the track $\gamma(i)$ with a path in $\widetilde{c_{i}}$ leading to its unique output is a track $\gamma^{i}$ of the single arc of $\bar{\Omega}$ contained in $L_{i}$. Clearly, $\mu_{\gamma^{i}}=\mu_{i}$ and $\gamma^{i}=\lambda_{i} \gamma_{i}$ where $\lambda_{i} \in \pi_{1}(E, z)$ is the longitude of $L_{i}$ determined by $\gamma(i)$ and the orientation and the framing of $L_{i}$. By definition of the surgery, the image of $\lambda_{i}$ in $\mathbf{X}$ represents $1 \in G$ and so $\bar{u}_{\gamma^{i}}=\varphi_{1}(\lambda(i))$. Then $(\bar{u})_{\gamma(i)}=(\bar{u})^{\gamma(i)}=\varphi_{1}(\lambda(i))$. By Section 5.3, the family of morphisms $\left\{\left(v^{1}\right)_{\gamma_{c}^{\prime}}:(\bar{u})_{\gamma_{c}^{\prime}} \rightarrow(\bar{u})^{\gamma_{c}^{\prime}}\right\}_{c} \cup\left\{\operatorname{id}_{\varphi_{1}(\lambda(i))}:(\bar{u})_{\gamma(i)} \rightarrow(\bar{u})^{\gamma(i)}\right\}_{i}$ determines a coupon-coloring $\bar{v}$ of $(\bar{\Omega}, \bar{u})$. Hence $(\bar{u}, \bar{v})$ is a $\mathcal{B}$-coloring of $\bar{\Omega}$. Denote the resulting $\mathcal{B}$-colored $G$-graph by $\bar{\Omega}^{\lambda}$. It follows from Section 5.3 that the isomorphism class of $\bar{\Omega}^{\lambda}$ does not depend on the choices of the tracks. Recall from TV4] the monoidal functor $F_{\mathcal{B}}$ from the category of isotopy classes of $\mathcal{B}$-colored $G$-graphs in $\mathbb{R}^{2} \times[0,1]$ to the category $\mathcal{B}$. In particular, this functor yields an isotopy invariant $F_{\mathcal{B}}\left(\bar{\Omega}^{\lambda}\right) \in$ $\operatorname{End}_{\mathcal{B}}(\mathbb{1})=\mathbb{k}$. Then

$$
\tau_{\mathcal{B}}(M, \Omega)=\Delta^{-n-1} \sum_{\lambda \in \operatorname{col}(L)}\left(\prod_{i=1}^{n} \operatorname{dim}(\lambda(i))\right) F_{\mathcal{B}}\left(\bar{\Omega}^{\lambda}\right) \in \mathbb{k}
$$


where $\Delta$ is the canonical rank of $\mathcal{B}$ defined in Section 2.7

8.3. The state-sum graph HQFT. Assume that $\mathbb{k}$ is a field and let $\mathcal{C}$ be a spherical $G$-fusion category over $\mathbb{k}$ with $\operatorname{dim}\left(\mathcal{C}_{1}\right) \neq 0$. In [TV2], we derive from $\mathcal{C}$ a 3 dimensional HQFT $|\cdot|_{\mathcal{C}}: \mathrm{Cob}^{G} \rightarrow \operatorname{Mod}_{\mathbb{k}}$ called the state sum HQFT. By Section 2.8, the $G$-center $\mathcal{Z}_{G}(\mathcal{C})$ of $\mathcal{C}$ is a $G$-ribbon (and so $G$-crossed) category over $\mathbb{k}$. In Section 10.3, we prove the following theorem:

Theorem 8.1. The state sum $H Q F T|\cdot|_{\mathcal{C}}$ extends to a graph $H Q F T$ over $\mathcal{Z}_{G}(\mathcal{C})$.

The proof of this theorem goes by extending the state sum method of TV2 to so-called knotted plexuses in skeletons (which represent ribbon graphs, see Section (10.1) via an invariant of colored knotted $G$-nets (see Section 9). The graph HQFT constructed in the proof of Theorem 8.1 is called the state sum graph HQFT and is again denoted by $|\cdot|_{\mathcal{C}}$.

8.4. Comparison theorem. Assume that $\mathbb{k}$ is an algebraically closed field and let $\mathcal{C}=\oplus_{g \in G} \mathcal{C}_{g}$ be an additive spherical $G$-fusion category over $\mathbb{k}$ with $\operatorname{dim}\left(\mathcal{C}_{1}\right) \neq 0$. By Section 2.8, the $G$-center $\mathcal{Z}_{G}(\mathcal{C})$ of $\mathcal{C}$ is an additive anomaly free $G$-modular category over $\mathbb{k}$. By Sections 8.2 and 8.3 , the category $\mathcal{C}$ gives rise to two graph HQFTs: the surgery graph HQFT $\tau_{\mathcal{Z}_{G}(\mathcal{C})}: \operatorname{Cob}_{\mathcal{Z}_{G}(\mathcal{C})}^{G} \rightarrow \operatorname{Mod}_{\mathbb{k}}$ and the state sum graph HQFT $|\cdot|_{\mathcal{C}}: \operatorname{Cob}_{\mathcal{Z}_{G}(\mathcal{C})}^{G} \rightarrow \operatorname{Mod}_{\mathbb{k}}$. Our main result is the following theorem:

Theorem 8.2. The graph HQFTs $\tau_{\mathcal{Z}_{G}(\mathcal{C})}$ and $|\cdot|_{\mathcal{C}}$ are isomorphic.

This theorem yields a surgery computation of $|\cdot| c$. For $G=\{1\}$, Theorem 8.2 was first established in [TV1] and independently (in the case char $(\mathbb{k})=0$ ) in $[\mathrm{Ba}$. We prove Theorem 8.2 in Section 12

By Section 8.1 any graph HQFT yields a scalar invariant of colored $G$-graphs in closed oriented 3-manifolds. The next claim directly follows from Theorem 8.2 .

Corollary 8.3. For any $\mathcal{Z}_{G}(\mathcal{C})$-colored $G$-graph $\Omega$ in a closed oriented 3-manifold $M$,

$$
\tau_{\mathcal{Z}_{G}(\mathcal{C})}(M, \Omega)=|M, \Omega|_{\mathcal{C}}
$$

The following corollary of Theorem 8.2 is Theorem 1.1 of the introduction:

Corollary 8.4. The surgery $H Q F T \tau_{\mathcal{Z}_{G}(\mathcal{C})}: \mathrm{Cob}^{G} \rightarrow \operatorname{Mod}_{\mathbb{k}}$ and the state sum $H Q F T|\cdot|_{\mathcal{C}}: \mathrm{Cob}^{G} \rightarrow \operatorname{Mod}_{\mathbb{k}}$ are isomorphic.

Proof. Both HQFTs extend to graph HQFTs which are isomorphic by Theorem 8.2 . Restricting the isomorphism in question to $\mathrm{Cob}^{G}$ we obtain an isomorphism of the original HQFTs.

Applying Corollary 8.3 to empty $G$-graphs, we get the following:

Corollary 8.5. For any closed 3-dimensional $G$-manifold $M$,

$$
\tau_{\mathcal{Z}_{G}(\mathcal{C})}(M)=|M|_{\mathcal{C}}
$$

Note finally that Theorem 8.2 and the non-degeneracy of the surgery graph HQFT $\tau_{\mathcal{Z}(\mathcal{C})}$ imply that the state sum graph HQFT $|\cdot|_{\mathcal{C}}$ is non-degenerate.

8.5. Remark. Using the language of higher categories, one can define extended HQFTs. Any graph HQFT induces a 2-extended 3-dimensional HQFT in the sense of [SW] with values in the 2-category of 2 -vector spaces. This observation uses the fact that the complement of a ribbon tangle with no coupons in a 3-manifold is a 3-manifold with corners of codimension 2. Theorem 8.2 implies that (under the assumptions of this theorem) the 2-extended 3-dimensional HQFTs induced by the graph HQFTs $|\cdot|_{\mathcal{C}}$ and $\tau_{\mathcal{Z}_{G}(\mathcal{C})}$ are isomorphic. 


\section{An inVARIANT OF COLORED KNOTTED NETS}

In this section, we define an invariant of colored knotted nets which generalizes $6 j$-symbols and which is used below to construct a state sum graph HQFT. Until the end of this section, we assume $\mathbb{k}$ to be a field and fix a $G$-fusion category $\mathcal{C}$ over $\mathbb{k}$. Recall that the $G$-center $\mathcal{Z}_{G}(\mathcal{C})$ of $\mathcal{C}$ is then a $G$-braided category over $\mathbb{k}$ (see Section 2.8). Note that all constructions of this section work word for word for a larger class of $G$-graded categories, namely for non-singular $G$-graded categories over arbitrary commutative rings (see Appendix A.1).

9.1. Knotted nets. We recall the notion of a knotted net in an oriented surface introduced in TV5, Section 15.3.2]. A net $\Gamma$ is a topological space obtained from a disjoint union of a finite number of oriented circles, oriented arcs, and oriented coupons (see Section 4.1) by gluing some endpoints of the arcs to the bases of the coupons or to each other. We require that different endpoints of the arcs are never glued to the same point of a (base of a) coupon. The images in $\Gamma$ of the arcs, half-arcs, circles, and coupons are called respectively edges, half-edges, circles, and coupons of $\Gamma$. The images in $\Gamma$ of the endpoints of the arcs that are not glued to coupons (but may be glued to each other) are called vertices of $\Gamma$. Every vertex of $\Gamma$ is incident to a certain number of half-edges of $\Gamma$, called the valency of the vertex. The edges and circles of $\Gamma$ are collectively called strands.

A knotted net $\Gamma$ in an oriented surface $\Sigma$ is a net immersed in $\Sigma \backslash \partial \Sigma$ such that

(i) all coupons of $\Gamma$ are embedded in $\Sigma$ preserving orientation;

(ii) all multiple points of the immersion are double transversal intersections of the interiors of strands of $\Gamma$. At every double point, one of the two meeting strands is distinguished.

Note that the coupons and the vertices of a knotted net are pairwise disjoint. The double points of a knotted net are called crossing points or just crossings. They are finite in number and lie away from the coupons and the vertices. A crossing of a knotted net in $\Sigma$ lies in an open disk in $\Sigma$ represented in our pictures by a plane parallel to the page. The orientation of $\Sigma$ is represented by the counterclockwise orientation of this plane. The distinguished strand at the crossing is represented by a red continuous (unbroken) line:

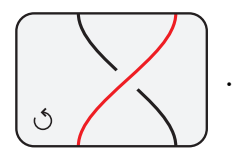

Each crossing $x$ of a knotted net $\Gamma$ in $\Sigma$ gives rise to two points on the strands of $\Gamma$ : the overcrossing $x_{\mathrm{ov}}$ lying in the distinguished strand $\left(x_{\mathrm{ov}}\right.$ is represented in our pictures by a red point) and the undercrossing $x_{\text {un }}$. The overcrossings split the strands of $\Gamma$ into consecutive segments called underpasses. A crossing $x$ of $\Gamma$ determines three underpasses: the underpass $\underline{x}$ containing the point $x_{\text {un }}$ and two underpasses $x^{-}, x^{+}$separated by the point $x_{\mathrm{ov}}$. One of the underpasses $x^{-}, x^{+}$ is directed towards $x_{\mathrm{ov}}$ and the other one is directed away from $x_{\mathrm{ov}}$. We choose notation so that $x^{+}$is directed towards $x_{\mathrm{ov}}$ if the crossing $x$ is positive and away from $x_{\mathrm{ov}}$ if $x$ is negative:
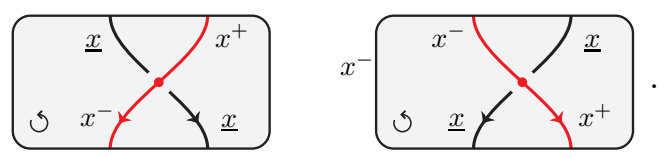

9.2. Colored knotted nets. A $\mathcal{C}$-coloring $U$ of a knotted net $\Gamma$ in an oriented surface $\Sigma$ comprises three functions. The first function assigns to each underpass $p$ of $\Gamma$ a homogeneous object $U_{p}$ of $\mathcal{C}$ or of $\mathcal{Z}_{G}(\mathcal{C})$, called the color of $p$. We require that 
if $p$ lies in a strand of $\Gamma$ which is incident to a coupon or is the distinguished strand of at least one crossing, then $U_{p} \in \mathcal{Z}_{G}(\mathcal{C})$. For any other $p$, either $U_{p} \in \mathcal{Z}_{G}(\mathcal{C})$ or $U_{p} \in \mathcal{C}$. The second function assigns to every crossing $x$ of $\Gamma$ an isomorphism

$$
U_{x}: U_{x^{+}} \rightarrow \varphi_{\left|U_{\underline{x}}\right|}\left(U_{x^{-}}\right)
$$

in $\mathcal{Z}_{G}(\mathcal{C})$ called the color of $x$. Here $\varphi$ is the crossing of the category $\mathcal{Z}_{G}(\mathcal{C})$ and, as usual, the degree in $G$ of a homogenous object $X$ of a $G$-graded category is denoted by $|X|$. If the colors involved are non-zero objects, then the existence of an isomorphism (10) implies that

$$
\left|U_{x^{+}}\right|=\left|U_{\underline{x}}\right|^{-1}\left|U_{x^{-}}\right|\left|U_{\underline{x}}\right| \in G .
$$

The third function assigns to each coupon $c$ of $\Gamma$ a morphism $c_{\text {in }} \rightarrow c^{\text {out }}$ in $\mathcal{Z}_{G}(\mathcal{C})$, where $c_{\text {in }}$ (respectively, $c^{\text {out }}$ ) is the object of $\mathcal{Z}_{G}(\mathcal{C})$ determined in the usual way by the colors and orientations of the inputs (respectively, outputs) of $c$. This morphism $c_{\text {in }} \rightarrow c^{\text {out }}$ is called the color of $c$.

A knotted $\mathcal{C}$-net is a knotted net endowed with a $\mathcal{C}$-coloring. Given a knotted $\mathcal{C}$-net $\Gamma$ in $\Sigma$, the subcolor of an underpass colored with an object $X$ is $X$ itself if $X \in \mathcal{C}$ and is the image $\mathcal{U}(X) \in \mathcal{C}$ of $X$ under the forgetful functor $\mathcal{U}: \mathcal{Z}_{G}(\mathcal{C}) \rightarrow \mathcal{C}$ if $X \in \mathcal{Z}_{G}(\mathcal{C})$. The subcolor of a crossing/coupon is the image of its color under the functor $\mathcal{U}$. Note that the above notion of a knotted $\mathcal{C}$-net generalizes the one in [TV5, Section 15.3.3] where $G=1$ and the crossings are not colored.

We will use the notions of a cyclic $\mathcal{C}$-set and its associated multiplicity module, see [TV5, Chapter 12]. A vertex $v$ of a knotted $\mathcal{C}$-net $\Gamma$ in $\Sigma$ determines a cyclic $\mathcal{C}$-set $\left(E_{v}, c_{v}, \varepsilon_{v}\right)$ as follows: $E_{v}$ is the set of half-edges of $\Gamma$ incident to $v$ with cyclic order induced by the opposite orientation of $\Sigma$, the map $c_{v}: E_{v} \rightarrow \mathrm{Ob}(\mathcal{C})$ assigns to a half-edge $e \in E_{v}$ the subcolor of the edge of $\Gamma$ containing $e$, and the map $\varepsilon_{v}: E_{v} \rightarrow\{+,-\}$ assigns to $e \in E_{v}$ the sign + if $e$ is oriented towards $v$ and - otherwise. Let $H_{v}(\Gamma)=H\left(E_{v}\right)$ be the multiplicity module of $E_{v}$. The $\mathbb{k}$-module $H_{v}(\Gamma)$ can be described as follows. Let $n \geq 1$ be the valence of $v$ and let $e_{1}<e_{2}<\cdots<e_{n}<e_{1}$ be the half-edges of $\Gamma$ incident to $v$ with cyclic order induced by the opposite orientation of $\Sigma$. Then we have the cone isomorphism

$$
\tau_{e_{1}}^{v}: H_{v}(\Gamma) \stackrel{\simeq}{\longrightarrow} \operatorname{Hom}_{\mathcal{C}}\left(\mathbb{1}, X_{1}^{\varepsilon_{1}} \otimes \cdots \otimes X_{n}^{\varepsilon_{n}}\right) .
$$

where $X_{r}=c_{v}\left(e_{r}\right)$ and $\varepsilon_{r}=\varepsilon_{v}\left(e_{r}\right)$ are the subcolor and sign of $e_{r}$ for all $r$. Set

$$
H(\Gamma)=\otimes_{v} H_{v}(\Gamma),
$$

where $v$ runs over all vertices of $\Gamma$ and $\otimes$ is the unordered tensor product of $\mathbb{k}$-modules. To emphasize the role of $\Sigma$, we sometimes write $H_{v}(\Gamma ; \Sigma)$ for $H_{v}(\Gamma)$ and $H(\Gamma ; \Sigma)$ for $H(\Gamma)$. If $\Gamma$ has no vertices, then by definition $H(\Gamma)=\mathbb{k}$.

An isotopy of knotted $\mathcal{C}$-nets in $\Sigma$ is an ambient isotopy in the class of knotted $\mathcal{C}$-nets in $\Sigma$ preserving all the data, that is the vertices, the strands, the crossings (with their distinguished strand), the coupons (with their distinguished base), the orientations, and the colors. An isotopy between two knotted $\mathcal{C}$-nets $\Gamma$ and $\Gamma^{\prime}$ in $\Sigma$ induces a $\mathbb{k}$-linear isomorphism $H(\Gamma) \rightarrow H\left(\Gamma^{\prime}\right)$ in the obvious way.

Any orientation preserving embedding $f$ of $\Sigma$ into an oriented surface $\Sigma^{\prime}$ carries a knotted $\mathcal{C}$-net $\Gamma$ in $\Sigma$ into a knotted $\mathcal{C}$-net $\Gamma^{\prime}=f(\Gamma)$ in $\Sigma^{\prime}$ and induces a $\mathbb{k}$-linear isomorphism $H(f): H(\Gamma ; \Sigma) \rightarrow H\left(\Gamma^{\prime} ; \Sigma^{\prime}\right)$ in the obvious way. This applies, in particular, when $f$ is an orientation preserving self-homeomorphism of $\Sigma$.

9.3. An invariant of knotted $\mathcal{C}$-nets in $\mathbb{R}^{2}$. Let $\Gamma$ be a knotted $\mathcal{C}$-net in the plane $\mathbb{R}^{2}$ (oriented counterclockwise). Pick a vector $\alpha_{v} \in H_{v}(\Gamma)$ for every vertex $v$ of $\Gamma$ and transform $\Gamma$ at its vertices, crossings, and coupons to obtain a Penrose diagram as follows. First, at each vertex $v$ of $\Gamma$, pick a half-edge $e_{v} \in E_{v}$, isotop $\Gamma$ near $v$ so that the half-edges incident to $v$ lie above $v$ with respect to the second 
coordinate on $\mathbb{R}^{2}$ and $e_{v}$ is the leftmost of them, and replace $v$ by a box colored with $\tau_{e_{v}}^{v}\left(\alpha_{v}\right)$, where $\tau^{v}$ is the universal cone of $H_{v}(\Gamma)$ :

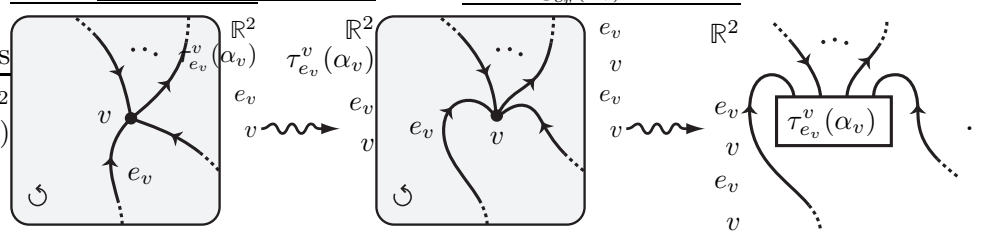

Next, at each crossing $x$ of $\Gamma$, isotop $\Gamma$ near $x$ to make to ensure that the strands are oriented downward. Consider the color $(A, \sigma) \in \mathcal{Z}_{G}(\mathcal{C})$ of the underpass $x^{+}$, the color $(B, \gamma) \in \mathcal{Z}_{G}(\mathcal{C})$ of the underpass $x^{-}$, the subcolor $X \in \mathcal{C}_{\text {hom }}$ of the underpass $\underline{x}$, and the subcolor $\psi: A \rightarrow \mathcal{U}\left(\varphi_{|X|}(B, \gamma)\right)$ of the crossing $x$. If the crossing $x$ is positive, then replace $x$ as follows:

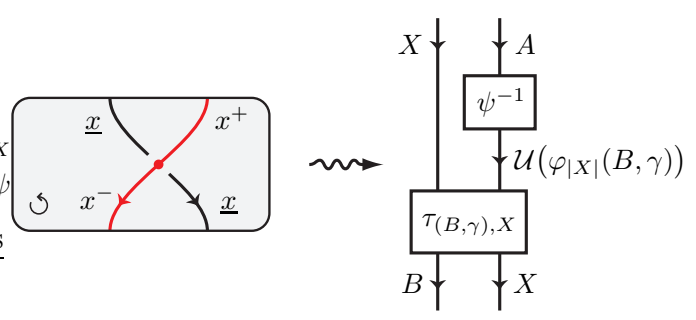

where $\tau$ is the enhanced $G$-braiding of $\mathcal{Z}_{G}(\mathcal{C})$, see Appendix A.4. If the crossing $x$ is negative, then replace $x$ as follows:

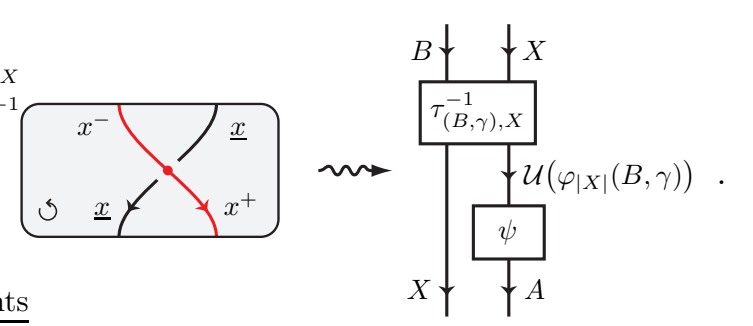

Finally, at each coupon $c$ of $\Gamma$, isotop $\Gamma$ near $c$ to make the bases of $c$ horizontal and to ensure that the distinguished (bottom) base lies below the opposite base with respect to the second coordinate on $\mathbb{R}^{2}$, replace $c$ by a box with the same inputs and outputs as $c$, and label this box with the subcolor of $c$ :

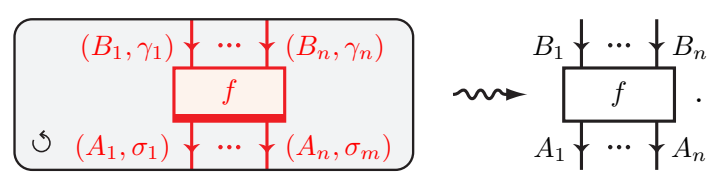

Also, the colors of all edges of $\Gamma$ are traded for the corresponding subcolors. This turns $\Gamma$ in a $\mathcal{C}$-colored Penrose diagram without free ends. The Penrose calculus associates with this diagram an element of $\operatorname{End}_{\mathcal{C}}(\mathbb{1})$ denoted $\mathbb{F}_{\mathcal{C}}(\Gamma)\left(\otimes_{v} \alpha_{v}\right)$. By linear extension, this procedure defines a $\mathbb{k}$-linear homomorphism

$$
\mathbb{F}_{\mathcal{C}}(\Gamma): H(\Gamma)=\otimes_{v} H_{v}(\Gamma) \rightarrow \operatorname{End}_{\mathcal{C}}(\mathbb{1})
$$

This homomorphism is an isotopy invariant of the $\mathcal{C}$-colored graph $\Gamma$. More precisely, for any isotopy $\iota$ between two knotted $\mathcal{C}$-nets $\Gamma$ and $\Gamma^{\prime}$ in $\mathbb{R}^{2}$, we have $\mathbb{F}_{\mathcal{C}}\left(\Gamma^{\prime}\right) H(\iota)=\mathbb{F}_{\mathcal{C}}(\Gamma)$, where $H(\iota): H(\Gamma) \rightarrow H\left(\Gamma^{\prime}\right)$ is the $\mathbb{k}$-linear isomorphism induced by $\iota$. 
9.4. An example. Let $\Gamma$ be the following knotted $\mathcal{C}$-net in $\mathbb{R}^{2}$ :

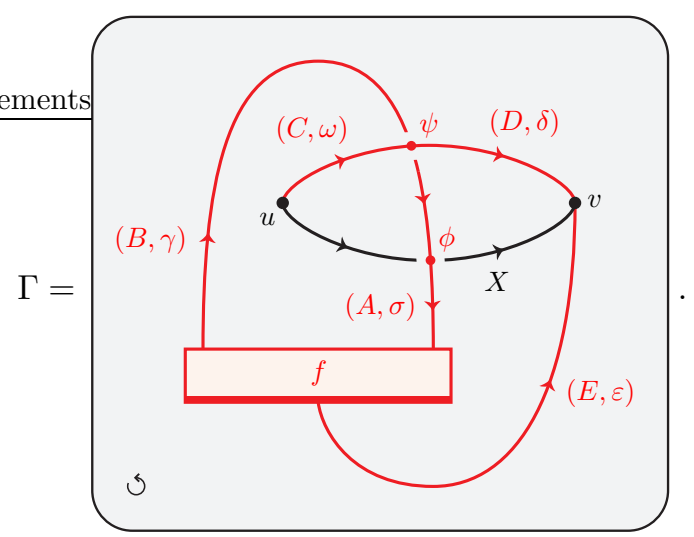

The net $\Gamma$ has two vertices $u$ and $v$, six underpasses colored $(A, \sigma),(B, \gamma),(C, \omega)$, $(D, \delta),(E, \varepsilon) \in \mathcal{Z}_{G}(\mathcal{C})_{\text {hom }}$ and $X \in \mathcal{C}_{\text {hom }}$, one coupon colored by a morphism in $\mathcal{Z}_{G}(\mathcal{C})$

$$
f:(E, \varepsilon) \rightarrow(B, \gamma)^{*} \otimes(A, \sigma)
$$

and two crossings colored by isomorphisms in $\mathcal{Z}_{G}(\mathcal{C})$

$$
\phi:(B, \gamma) \rightarrow \varphi_{|X|}(A, \sigma) \text { and } \psi:(D, \delta) \rightarrow \varphi_{|B|}(C, \omega) .
$$

Clearly, $H(\Gamma)=H_{u}(\Gamma) \otimes H_{v}(\Gamma)$. Pick $\alpha \in H_{u}(\Gamma)$ and $\beta \in H_{v}(\Gamma)$. Then, by definition, we have

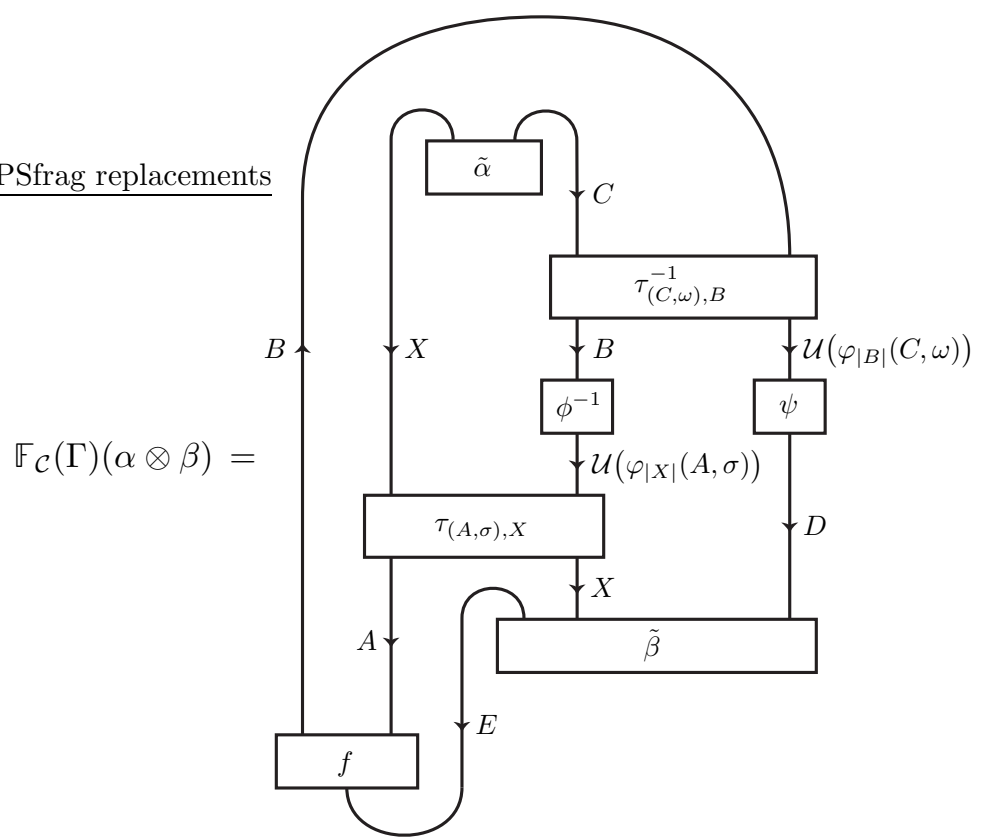

where $\tilde{\alpha}$ and $\tilde{\beta}$ are the ismages of $\alpha$ and $\beta$ under the cone isomorphisms

$$
H_{u}(\Gamma) \stackrel{\simeq}{\longrightarrow} \operatorname{Hom}_{\mathcal{C}}\left(\mathbb{1}, X^{*} \otimes C^{*}\right) \text { and } H_{v}(\Gamma) \stackrel{\simeq}{\longrightarrow} \operatorname{Hom}_{\mathcal{C}}(\mathbb{1}, E \otimes X \otimes D) .
$$

9.5. An invariant of knotted $\mathcal{C}$-nets in 2-spheres. Suppose that the category $\mathcal{C}$ is spherical. Then the invariant $\mathbb{F}_{\mathcal{C}}$ of knotted $\mathcal{C}$-nets in $\mathbb{R}^{2}$ defined in Section 9.3 extends uniquely to an isotopy invariant of knotted $\mathcal{C}$-nets in the 2 -sphere $S^{2}=\mathbb{R}^{2} \cup$ $\{\infty\}$ endowed with the orientation extending the counterclockwise orientation in $\mathbb{R}^{2}$. Indeed, consider a knotted $\mathcal{C}$-net $\Gamma$ in $S^{2}$. Pushing $\Gamma$ away from $\infty$ by an isotopy, we obtain a $\mathcal{C}$-colored graph $\Gamma_{0}$ in $\mathbb{R}^{2}$. The isotopy induces a $\mathbb{k}$-linear isomorphism 
$H\left(\Gamma ; S^{2}\right) \simeq H\left(\Gamma_{0} ; \mathbb{R}^{2}\right)$. Composing with $\mathbb{F}_{\mathcal{C}}\left(G_{0}\right): H\left(\Gamma_{0} ; \mathbb{R}^{2}\right) \rightarrow \operatorname{End}_{\mathcal{C}}(\mathbb{1})$ we obtain a $\mathbb{k}$-linear homomorphism

$$
\mathbb{F}_{\mathcal{C}}(\Gamma): H\left(\Gamma ; S^{2}\right) \rightarrow \operatorname{End}_{\mathcal{C}}(\mathbb{1}) .
$$

The sphericity of $\mathcal{C}$ implies that this homomorphism does not depend on the way we push $\Gamma$ in $\mathbb{R}^{2}$ and is an isotopy invariant of $\Gamma$.

The invariant $\mathbb{F}_{\mathcal{C}}$ further extends to knotted $\mathcal{C}$-nets in an arbitrary oriented surface $\Sigma$ homeomorphic to $S^{2}$. Namely, given a knotted $\mathcal{C}$-net $\Gamma$ in $\Sigma$, pick an orientation preserving homeomorphism $f: \Sigma \rightarrow S^{2}$, consider the induced $\mathbb{k}$-linear isomorphism $H(f): H(\Gamma ; \Sigma) \rightarrow H\left(f(\Gamma) ; S^{2}\right)$, and set

$$
\mathbb{F}_{\mathcal{C}}(\Gamma)=\mathbb{F}_{\mathcal{C}}(f(\Gamma)) \circ H(f): H(\Gamma ; \Sigma) \rightarrow \operatorname{End}_{\mathcal{C}}(\mathbb{1}) .
$$

Since all orientation preserving homeomorphisms $\Sigma \rightarrow S^{2}$ are isotopic, this homomorphism does not depend on the choice of $f$ and is an isotopy invariant of $\Gamma$.

We view $\mathbb{F}_{\mathcal{C}}(\Gamma)$ as a generalization of the familiar $6 j$-symbols which arise when $\Gamma \subset S^{2}$ is the 1-skeleton of a tetrahedron.

\section{The STATE SUm Graph HQFT}

In this section we construct state sum graph HQFTs over the $G$-centers of spherical $G$-fusion categories. This construction is based on a presentation of ribbon graphs in 3-manifolds by knotted plexuses in skeletons introduced in TV5, Chapter 14] where we refer for details.

10.1. Knotted plexuses in skeletons. Let $M$ be a closed oriented 3-manifold. A skeleton of $M$ is an oriented compact 2-dimensional complex $P \subset M$ whose complement in $M$ is a disjoint union of open 3-balls called the $P$-balls. A vertex of $P$ is a point of the 0 -skeleton $P^{(0)}$ of $P$. We let $P^{(1)}$ be the 1 -skeleton of $P$. Here $P$ oriented means that the surface $\operatorname{Int}(P)=P \backslash P^{(1)}$ is oriented. The set $P^{(1)} \backslash P^{(0)}$ is a finite disjoint union of open intervals whose closures are called the edges of $P$.

By a plexus, we mean a topological space obtained from a disjoint union of a finite number of arcs and coupons by gluing the endpoints of the arcs to the bases of the coupons. We require that different endpoints of the arcs are never glued to the same point of a (base of a) coupon.

A knotted plexus $d$ in $P$ is a plexus drawn (i.e., immersed) in $P \backslash P^{(0)}$ possibly with double crossings of $\operatorname{arcs}$ in $\operatorname{Int}(P)$ so that at every crossing, one of two arcs is distinguished. All coupons of $d$ must lie in $\operatorname{Int}(P)$ while the arcs of $d$ may meet the edges of $P$ transversely at a finite number of points called the switches of $d$. A neighborhood in $P$ of a switch of $d$ is formed by a finite number (greater than or equal to 2) of half-planes adjacent to the edge of $P$ containing the switch so that the plexus $d$ meets these half-planes along a segment contained in the union of two of them:

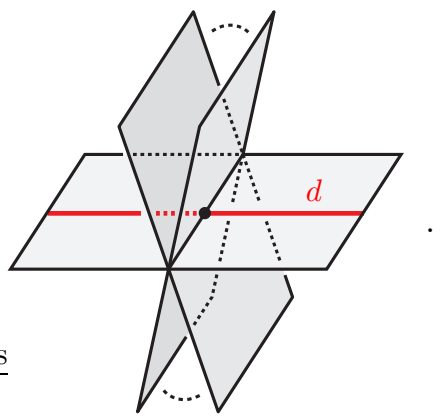


We assume that the given orientations of the regions of $P$ containing these two half-planes are compatible near the switch, i.e., they are induced by an orientation of the horizontal plane in the above figure. (The pair $(P, d)$ is called a positive diagram without circle components in TV5.)

Every knotted plexus $d$ in $P$ determines a ribbon graph in $M$ as follows. Pick a field of normal directions $n$ on $\operatorname{Int}(P)$ such that the orientation of $\operatorname{Int}(P)$ followed by $n$ yields the opposite orientation of $M$. Slightly pushing the undistinguished strands at the crossings of $d$ along $n$, we obtain an embedding $d \hookrightarrow M$ whose image is denoted $d^{n}$. The above orientation condition at the switches implies that the vector field $n$ can be chosen to continuously extend to all switches and to determine thus a framing of $d^{n}$. This turns $d^{n}$ into a ribbon graph in $M$. We say that a ribbon graph in $M$ is represented by $d$ if it is isotopic to $d^{n}$.

By [TV5, Corollary 14.5], all ribbon graphs in $M$ can be represented by knotted plexus in skeletons of $M$, and two knotted plexus in skeletons of $M$ represent isotopic ribbon graphs if they can be related by certain moves generalizing the Reidemeiter moves to skeletons.

10.2. An invariant of colored $G$-graphs. In the rest of this section, $\mathcal{C}$ is a spherical $G$-fusion category over a field $\mathbb{k}$ such that $\operatorname{dim}\left(\mathcal{C}_{1}\right) \neq 0$. Recall from Section 2.8 that the $G$-center $\mathcal{Z}_{G}(\mathcal{C})$ of $\mathcal{C}$ is then a $G$-ribbon category. We define here a state sum invariant $|M, \Omega|_{\mathcal{C}} \in \mathbb{k}$ for any $\mathcal{Z}_{G}(\mathcal{C})$-colored $G$-graph $\Omega$ in a closed oriented 3-manifold $M$.

Let $P$ be a skeleton of $M$ and $d$ be a knotted plexus in $P$ representing the underlying ribbon graph of $\Omega$ (see Section 10.1). The vertices of $P$ and the switches, crossings, and coupons of $d$ are called the nodes of $d$. The complement of the nodes in $\tilde{d}=d \cup P^{(1)} \subset P$ is a finite disjoint union of open intervals. Their closures are called the rims of $d$. Each rim $e$ lies in $P^{(1)}$ or in $d$ and connects two nodes (possibly, equal) called the endpoints of $e$. By an oriented rim we mean a rim endowed with orientation (which may be compatible or not with the orientation of the strand of $d$ containing this rim). Cutting $P$ along $\tilde{d}$, we obtain a compact surface (with interior $P \backslash \tilde{d}$ ) whose components are called the faces of $d$. We let $\operatorname{Fac}(d)$ be the (finite) set of faces of $d$.

We define a map $\ell: \operatorname{Fac}(d) \rightarrow G$, called the $G$-labeling of $d$. In every $P$-ball, pick a point, called its center. Pick in the given homotopy class of maps $\left(M \backslash \Omega, \Omega_{\bullet}\right) \rightarrow$ $(\mathbf{X}, \mathbf{x})$ a representative $g: M \backslash \Omega \rightarrow \mathbf{X}$ carrying the centers of all $P$-balls to $\mathbf{x}$. Clearly, each face $r$ of $d$ is adjacent to two (possibly coinciding) $P$-balls. Pick an oriented arc in $M$ connecting the centers of these balls and meeting $P$ transversely in a single point lying in the interior of $r$. We orient this arc so that its intersection number with the face $r$ is equal to +1 . Applying $g$ to the resulting oriented arc we get a loop in $\mathbf{X}$ representing $\ell(r) \in G=\pi_{1}(\mathbf{X}, \mathbf{x})$.

We now use the given $\mathcal{Z}_{G}(\mathcal{C})$-coloring of the $G$-graph $\Omega$ to color the coupons and the crossings of $d$, as well as the rims of $d$ lying in $d$. To this end, pick a 1 -system of tracks $\left\{\gamma_{\lambda}\right\}_{\lambda}$ and a 2-system of tracks $\left\{\gamma_{c}\right\}_{c}$ for $\Omega$, where $\lambda$ runs over the arcs and $c$ runs over the coupons of $\Omega$. First, consider a rim $e \subset d$. Slightly pushing $e$ along the framing of $\Omega$, we obtain a parallel copy $\widetilde{e}$ of $e$ lying inside a $P$-ball. Let $\delta_{e}$ be a path in this $P$-ball leading from its center to $\widetilde{e}$. Let $\lambda=\lambda_{e}$ be the arc of $\Omega$ supporting $e$. Then, up to homotopy in $M \backslash \Omega$, we have $\delta_{e}=\alpha_{e} \gamma_{\lambda}$ for a unique homotopy class of paths $\alpha_{e}$ in $M \backslash \Omega$ leading from the center of the $P$-ball above to the point $\gamma_{\lambda}(0) \in \Omega_{\bullet}$. The map $g$ carries $\alpha_{e}$ to an element of $G=\pi_{1}(\mathbf{X}, \mathbf{x})$ again

\footnotetext{
${ }^{3}$ More generally, the constructions of Section 10 work when $\mathcal{C}$ is a spherical $G$-fusion category over a commutative ring such that $\operatorname{dim}\left(\mathcal{C}_{1}\right)$ is invertible and all idempotents in $\mathcal{C}$ split (see Appendix A.1). Indeed, in this case, $\mathcal{Z}_{G}(\mathcal{C})$ is also a $G$-ribbon category.
} 
denoted $\alpha_{e}$. The given precoloring $u$ of $\Omega$ yields an object $u_{\gamma_{\lambda}} \in \mathcal{Z}_{G}(\mathcal{C})$. The color of $e$ is the object

$$
U_{e}=\varphi_{\alpha_{e}^{-1}}\left(u_{\gamma_{\lambda}}\right) \in \mathcal{Z}_{G}(\mathcal{C})
$$

which is homogeneous of degree $\alpha_{e} \mu_{\gamma_{\lambda}} \alpha_{e}^{-1} \in G$. Next, consider a crossing $x$ of $d$. Let $e^{+}, e^{-}$be the rims adjacent to $x$ and lying in the distinguished strand of $x$. We choose notation so that $e^{+}$is directed towards $x$ if the crossing $x$ is positive and away from $x$ if $x$ is negative:
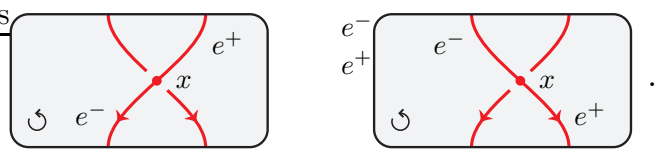

The color of $x$ is the isomorphism

$$
U_{x}=\left(\varphi_{2}\left(\alpha_{e^{-}} \alpha_{e^{+}}^{-1}, \alpha_{e^{-}}^{-1}\right)_{u_{\gamma_{\lambda}}}\right)^{-1}: U_{e^{+}} \rightarrow \varphi_{\alpha_{e^{-}} \alpha_{e^{+}}^{-1}}\left(U_{e^{-}}\right),
$$

where $\lambda$ is the arc of $\Omega$ supporting $e^{+} \cup e^{-}$. Finally, consider a coupon $c$ of $d$. Pushing $c$ along the framing of $\Omega$, we obtain a parallel copy $\widetilde{c}$ of $c$ lying inside a $P$-ball $B_{c}$. Let $\delta_{c}$ be a path in $B_{c}$ leading from its center to $\widetilde{c}$. Then up to homotopy in $M \backslash \Omega$, we have $\delta_{c}=\alpha_{c} \gamma_{c}$ for a unique homotopy class of paths $\alpha_{c}$ in $M \backslash \Omega$ leading from the center of $B_{c}$ to the point $\gamma_{c}(0) \in \Omega_{\bullet}$. Let $m \geq 0$ be the number of inputs of $c$ and let $e_{k}$ be the rim of $d$ incident to the $k$-th input for $k=1, \ldots, m$. Set $\varepsilon_{k}=+$ if $e_{k}$ is directed out of $c$ at the $k$-th input and $\varepsilon_{k}=-$ otherwise. Let $\lambda_{k}$ be the arc of $\Omega$ supporting $e_{k}$ and set $\gamma_{k}=\gamma_{\lambda_{k}}$. Composing $\delta_{c}$ with a path in $\widetilde{c}$ leading to the $k$-th input, we obtain a path $\delta_{k}$ in $B_{c}$ which expands uniquely as $\delta_{k}=\alpha_{k} \gamma_{k}$ for a unique homotopy class of paths $\alpha_{k}$ in $M \backslash \Omega$ leading from the center of $B_{c}$ to the point $\gamma_{k}(0)=\gamma_{c}(0) \in \Omega_{\bullet}$. By definition, the color of the $\operatorname{rim} e_{k}$ is

$$
U_{e_{k}}=\varphi_{\alpha_{k}^{-1}}\left(u_{\gamma_{k}}\right) \in \mathcal{Z}_{G}(\mathcal{C}) \text {. }
$$

Similarly, let $n \geq 0$ be the number of outputs of $c$ and let $e^{l}$ be the arc of $\Omega$ incident to the $l$-th output for $l=1, \ldots, n$. Set $\varepsilon^{l}=+$ if $e^{l}$ is directed into $c$ at the $l$-th output and $\varepsilon^{l}=-$ otherwise. Let $\lambda^{l}$ be the arc of $\Omega$ supporting $e^{l}$ and set $\gamma^{l}=\gamma_{\lambda^{l}}$. Composing $\delta_{c}$ with a path in $\widetilde{c}$ leading to the $l$-th output, we obtain a path $\delta^{l}$ in $B_{c}$ which expands uniquely as $\delta^{l}=\alpha^{l} \gamma^{l}$ for a unique homotopy class of paths $\alpha^{l}$ in $M \backslash \Omega$ leading from the center of $B_{c}$ to the point $\gamma^{l}(0)=\gamma_{c}(0) \in \Omega_{\bullet}$. By definition, the color of the rim $e^{l}$ is

$$
U_{e^{l}}=\varphi_{\left(\alpha^{l}\right)^{-1}}\left(u_{\gamma^{l}}\right) \in \mathcal{Z}_{G}(\mathcal{C}) .
$$

The given coupon-coloring $v$ of $\Omega$ yields the morphism

$$
v_{\gamma_{c}}: u_{\gamma_{c}}=\bigotimes_{k=1}^{m} u_{\rho_{k}}^{\varepsilon_{k}} \longrightarrow u^{\gamma_{c}}=\bigotimes_{\ell=1}^{n} u_{\rho^{\ell}}^{\varepsilon^{\ell}}
$$

where $\rho_{k}$ is the composition of $\gamma_{c}$ with a path in $\widetilde{c}$ leading to the $k$-th input and $\rho^{l}$ is the composition of $\gamma_{c}$ with a path in $\widetilde{c}$ leading to the $l$-th output. Note that

$$
\rho_{k}=\alpha_{c}^{-1} \delta_{k}=\alpha_{c}^{-1} \alpha_{k} \gamma_{k} \quad \text { and } \quad \rho_{l}=\alpha_{c}^{-1} \delta^{l}=\alpha_{c}^{-1} \alpha^{l} \gamma^{l} .
$$

For a track $\gamma$ of an arc incident to $c$ and a homotopy class of paths $\alpha$ in $M \backslash \Omega$ leading from the center of $B_{c}$ to the point $\gamma(0)=\gamma_{c}(0) \in \Omega_{\bullet}$, the precoloring $u$ yields an isomorphism $u_{\alpha_{c}^{-1} \alpha, \gamma}: u_{\alpha_{c}^{-1} \alpha \gamma} \rightarrow \varphi_{\alpha^{-1} \alpha_{c}}\left(u_{\gamma}\right)$. Consider the isomorphisms

$$
\psi(\alpha, \gamma,+)=\varphi_{2}\left(\alpha_{c}^{-1}, \alpha^{-1} \alpha_{c}\right)_{u_{\gamma}} \circ \varphi_{\alpha_{c}^{-1}}\left(u_{\alpha_{c}^{-1} \alpha, \gamma}\right): \varphi_{\alpha_{c}^{-1}}\left(u_{\alpha_{c}^{-1} \alpha \gamma}\right) \rightarrow \varphi_{\alpha^{-1}}\left(u_{\gamma}\right) .
$$

and

$$
\psi(\alpha, \gamma,-)=\left(\psi(\alpha, \gamma,+)^{-1}\right)^{*} \circ \varphi_{\alpha_{c}^{-1}}^{1}\left(u_{\alpha_{c}^{-1} \alpha \gamma}\right): \varphi_{\alpha_{c}^{-1}}\left(u_{\alpha_{c}^{-1} \alpha \gamma}^{*}\right) \rightarrow \varphi_{\alpha^{-1}}\left(u_{\gamma}\right)^{*}
$$


The color of the coupon $c$ is the morphism

$$
U_{c}: \bigotimes_{k=1}^{m} U_{e_{k}}^{\varepsilon_{k}} \longrightarrow \bigotimes_{\ell=1}^{n} U_{e^{\varepsilon^{\ell}}}
$$

in $\mathcal{Z}_{G}(\mathcal{C})$ defined as the following composition:

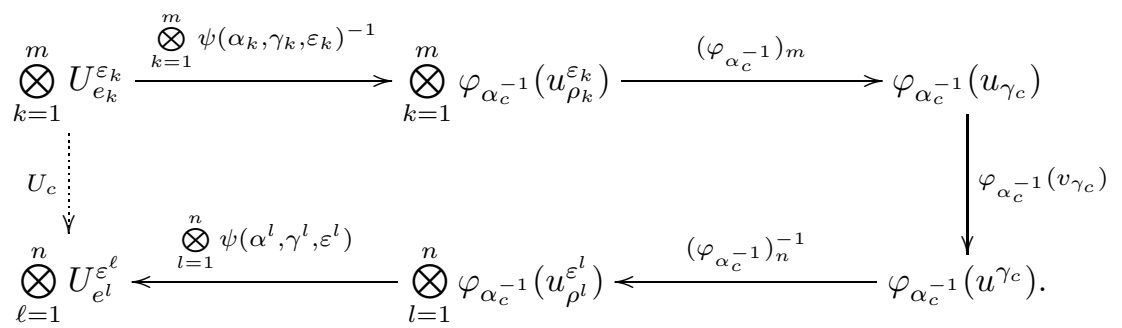

Fix from now on a representative set $I=\amalg_{\alpha \in G} I_{\alpha}$ of simple objects of $\mathcal{C}$. A $G$ coloring of $d$ is a map $\mathbf{c}: \operatorname{Fac}(d) \rightarrow I$ such that $\mathbf{c}(r) \in I_{\ell(r)}$ for all faces $r$ of $d$. The object $\mathbf{c}(r)$ assigned to a face $r$ of $d$ is called the $\mathbf{c}$-color of $r$. For each $G$-coloring $\mathbf{c}$ of $d$, we define a scalar $|\mathbf{c}| \in \mathbb{k}$ as follows.

First, for each oriented rim $e$ of $d$, we define a cyclic $\mathcal{C}$-set $P_{e, \mathbf{c}}$. If $e \subset P^{(1)}$, then $P_{e, \mathbf{c}}$ is the set of germs of faces of $d$ adjacent to $e$ turned into a cyclic $\mathcal{C}$-set as in [TV5, Section 13.1.1] using c. Explicitly, the orientations of $e$ and $M$ determine a positive direction on a small loop in $M$ encircling $e$. The resulting oriented loop determines a cyclic order on the set $P_{e, \mathbf{c}}$ of germs of faces of $d$ adjacent to $e$. To each $b \in P_{e, \mathbf{c}}$ we assign the c-color of the face of $d$ containing $b$ and a sign equal to + if the orientation of $b$ induces the one of $e \subset \partial b$ (that is, the orientation of $b$ is given by the orientation of $e$ followed by a vector at a point of $e$ directed inside $b$ ) and equal to - otherwise. In this way, $P_{e, \mathbf{c}}$ becomes a cyclic $\mathcal{C}$-set. If $e \subset d$, then $e$ is adjacent to two faces $r_{+}, r_{-}$of $d$ such that the orientation of $r_{+}$(induced by the one of $P$ ) induces the given orientation of $e$ and the orientation of $r_{-}$induces the opposite orientation of $e$ :

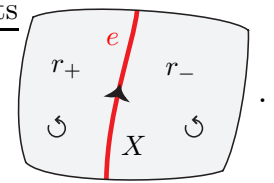

In this picture the arrow on $e$ indicates the orientation of $e$. Set $\varepsilon=+$ if the orientation of the strand of $d$ containing $e$ is compatible with that of $e$ and set $\varepsilon=-$ otherwise. Then $P_{e, \mathbf{c}}=\left\{r_{-}, e, r_{+}\right\}$where $r_{-}<e<r_{+}<r_{-}$and the map $\left\{r_{-}, e, r_{+}\right\} \rightarrow \mathrm{Ob}(\mathcal{C}) \times\{+,-\}$ carries $r_{ \pm}$to $\left(\mathbf{c}\left(r_{ \pm}\right), \pm\right)$and carries $e$ to $(X, \varepsilon)$ where $X \in \mathcal{C}$ is the image of the color of $e$ under the forgetful functor $\mathcal{Z}_{G}(\mathcal{C}) \rightarrow \mathcal{C}$.

For any oriented rim $e$ of $d$, let $H_{\mathbf{c}}(e)=H\left(P_{e, \mathbf{c}}\right)$ be the multiplicity module of $P_{e, \mathbf{c}}$. In particular, if $e \subset d$, then in the notation above

$$
H_{\mathbf{c}}(e) \simeq \operatorname{Hom}_{\mathcal{C}}\left(\mathbb{1}, c\left(r_{-}\right)^{*} \otimes X^{\varepsilon} \otimes c\left(r_{+}\right)\right)
$$

We let

$$
H_{\mathbf{c}}=\bigotimes_{e} H_{\mathbf{c}}(e)
$$

be the unordered tensor product of the $\mathbb{k}$-modules $H_{\mathbf{c}}(e)$ over all oriented rims $e$ of $d$. An unoriented rim $E$ of $d$ gives rise to two opposite oriented rims $e_{1}, e_{2}$ whose associated cyclic $\mathcal{C}$-sets $P_{e_{1}, \mathbf{c}}$ and $P_{e_{2}, \mathbf{c}}$ are dual to each other. This determines a contraction vector $*_{E} \in H_{\mathbf{c}}\left(e_{1}\right) \otimes H_{\mathbf{c}}\left(e_{2}\right)$, see [TV5, Section 12.3.4]. Set

$$
*_{\mathbf{c}}=\bigotimes_{E} *_{E} \in H_{\mathbf{c}}
$$


where $E$ runs over all unoriented rims of $d$.

We associate with each node $x$ of $d$ a knotted $\mathcal{C}$-net $\Gamma_{x}^{\mathbf{c}}$ in an oriented surface homeomorphic to $S^{2}$. We do it as in [TV5, Section 15.5.1] by appropriately incorporating colors to crossings of the involved knotted $\mathcal{C}$-nets. More precisely, we consider four cases, as follows.

(i) Let $x$ be a vertex of $P$. Pick a small closed ball neighborhood $B_{x} \subset M$ of $x$ such that $\Gamma_{x}=P \cap \partial B_{x}$ is a non-empty graph and $P \cap B_{x}$ is the cone over $\Gamma_{x}$ with summit $x$. The vertices of $\Gamma_{x}$ are the intersection points of the 2-sphere $\partial B_{x}$ with the edges of $P$ incident to $x$. The edges of $\Gamma_{x}$ are the intersections of $\partial B_{x}$ with the faces of $d$ adjacent to $x$. We endow $\partial B_{x}$ with the orientation induced by that of $M$ restricted to $M \backslash \operatorname{Int}\left(B_{x}\right)$. Every edge $a$ of $\Gamma_{x}$ lies in a face $r_{a}$ of $d$. We color $a$ with $\mathbf{c}\left(r_{a}\right) \in I$ and endow $a$ with the orientation induced by that of $r_{a} \backslash \operatorname{Int}\left(B_{x}\right)$. In this way, $\Gamma_{x}$ yields a $\mathcal{C}$-colored graph in $\partial B_{x}$ denoted $\Gamma_{x}^{\mathbf{c}}$. For example:

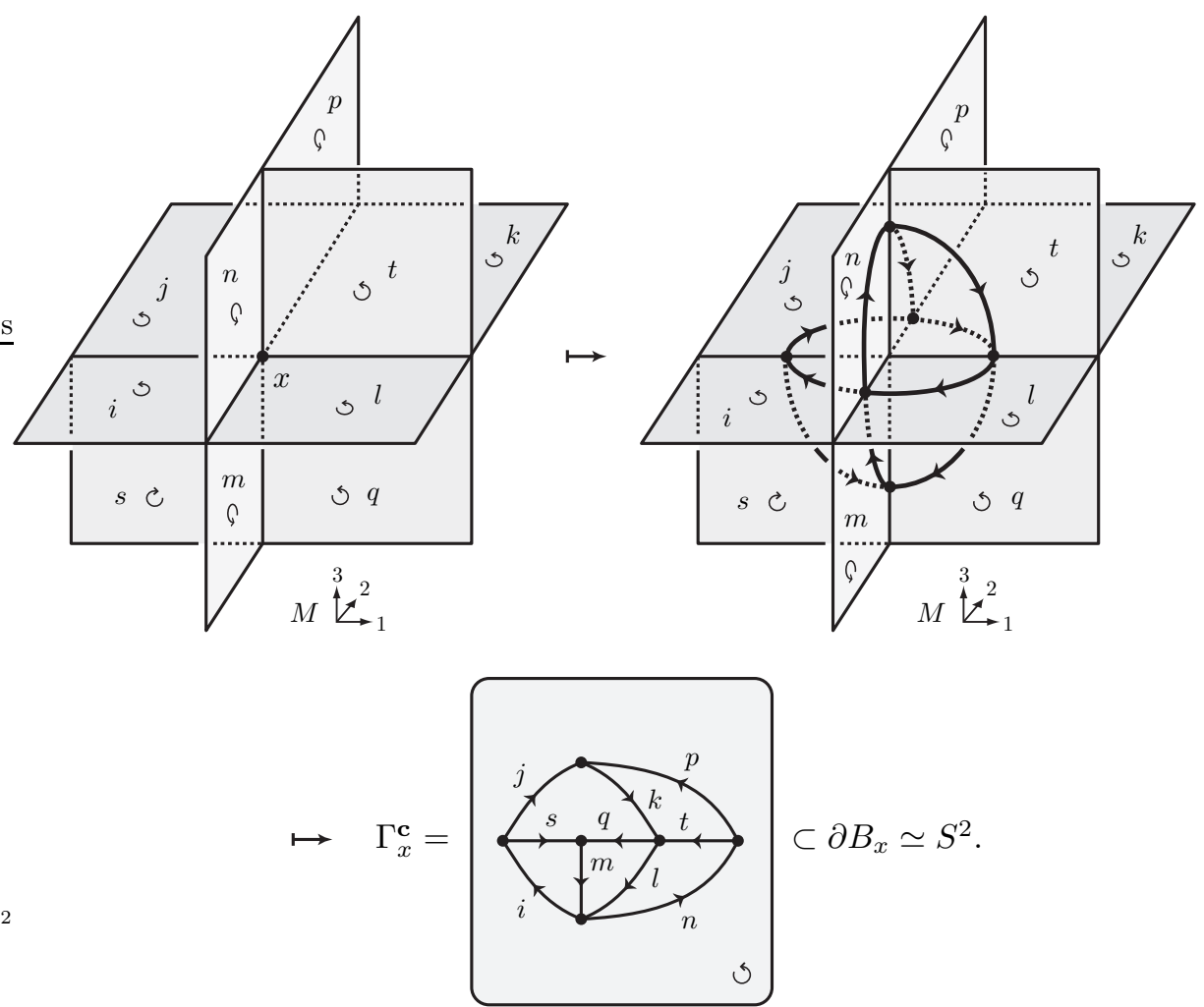


(ii) Let $x$ be a switch of $d$. A neighborhood of $x$ in $P$ looks as follows:

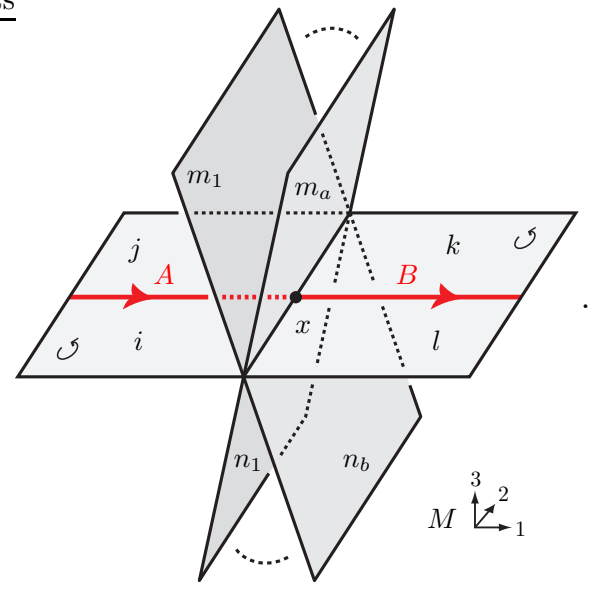

Here the orientation of $M$ is right-handed (as always in our pictures), the object $A=U_{e} \in \mathcal{Z}_{G}(\mathcal{C})$ is the color of the rim $e$ of $d$ directed to $x$, the object $B=U_{o} \in \mathcal{Z}_{G}(\mathcal{C})$ is the color of the rim $o$ of $d$ directed away from $x$, and $i, j, k, l, m_{1}, \ldots, m_{a}, n_{1}, \ldots, n_{b} \in I$ are the $\mathbf{c}$-colors of the faces of $d$ adjacent to $x$ with $a \geq 0$ and $b \geq 0$. By definition, $A=\varphi_{\alpha_{e}^{-1}}(X)$ and $B=\varphi_{\alpha_{o}^{-1}}(X)$, where $X=u_{\gamma_{\lambda}} \in \mathcal{Z}_{G}(\mathcal{C})$ is the evaluation of the precoloring $u$ of $\Omega$ on the track $\gamma_{\lambda}$ of the arc $\lambda$ of $\Omega$ supporting the rims $e$ and $o$. Set

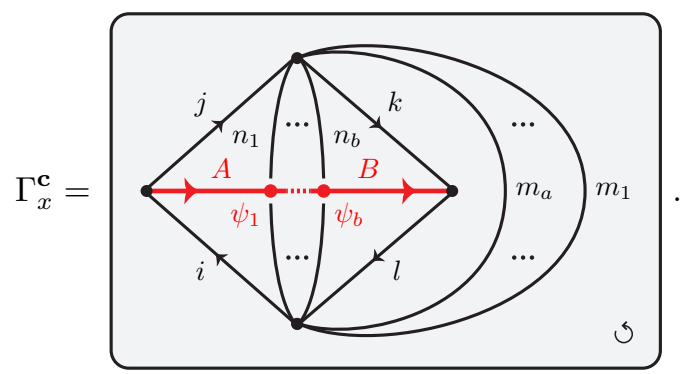

Here we draw $\Gamma_{x}^{\mathbf{c}}$ in the plane $\mathbb{R}^{2}$ (oriented counterclockwise) and view $\Gamma_{x}^{\mathbf{c}}$ as a knotted $\mathcal{C}$-net in $S^{2}=\mathbb{R}^{2} \cup\{\infty\}$. We direct the arc of $\Gamma_{x}^{\mathbf{c}}$ colored by $m_{p}$ with $1 \leq p \leq a$ upward if the orientation of the $m_{p}$-labeled face of $d$ followed by that of $d$ at $x$ yields the positive orientation of $M$, and downward otherwise. For $1 \leq q \leq b$, let $r_{q}$ be the $n_{q}$-labeled face of $d$. Set $\varepsilon_{q}=1$ if the orientation of $r_{q}$ followed by that of $d$ at $x$ yields the positive orientation of $M$ and set $\varepsilon_{q}=-1$ otherwise. We direct the arc of $\Gamma_{x}^{\mathbf{c}}$ colored by $n_{q}$ downward if $\varepsilon_{q}=1$ and upward otherwise. We numerate the $b+1$ arcs lying on the horizontal segment from left to right by $q=0,1, \ldots, b$ and color the $q$-th arc with

$$
A_{q}=\varphi_{\beta_{q}}(X) \in \mathcal{Z}_{G}(\mathcal{C}) \quad \text { where } \quad \beta_{q}=\alpha_{e}^{-1} \ell\left(r_{1}\right)^{\varepsilon_{1}} \cdots \ell\left(r_{q}\right)^{\varepsilon_{q}} \in G .
$$

Note that $A_{0}=A$ (since $\beta_{0}=\alpha_{e}^{-1}$ ) and $A_{b}=B$ (since $\beta_{b}=\alpha_{o}^{-1}$ ). The knotted net $\Gamma_{x}^{\mathbf{c}}$ has $b$ crossing points. We numerate them from left to right by $q=1, \ldots, b$ and color the $q$-th crossing with the isomorphism

$$
\psi_{q}= \begin{cases}\left(\varphi_{2}\left(\ell\left(r_{q}\right), \beta_{q-1}\right)_{X}\right)^{-1}: A_{q} \rightarrow \varphi_{\ell\left(r_{q}\right)}\left(A_{q-1}\right) & \text { if } \varepsilon_{q}=1, \\ \left(\varphi_{2}\left(\ell\left(r_{q}\right), \beta_{q}\right)_{X}\right)^{-1}: A_{q-1} \rightarrow \varphi_{\ell\left(r_{q}\right)}\left(A_{q}\right) & \text { if } \varepsilon_{q}=-1 .\end{cases}
$$


(iii) Let $x$ be a coupon of $d$ with $m \geq 0$ inputs and $n \geq 0$ outputs. A neighborhood of $x$ in $P$ looks as follows:

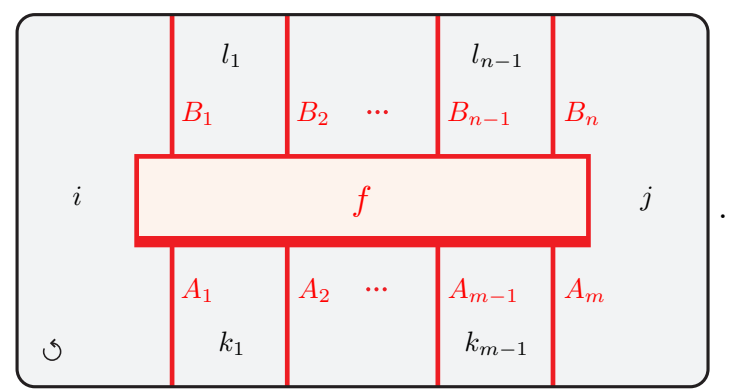

Here $i, j, k_{1}, \ldots, k_{m-1}, l_{1}, \ldots, l_{n-1} \in I$ are the c-colors of the faces of $d$ adjacent to the coupon, the objects $A_{1}, \ldots, A_{m} \in \mathcal{Z}_{G}(\mathcal{C})$ are the colors of the rims corresponding to the inputs, the objects $B_{1}, \ldots, B_{n} \in \mathcal{Z}_{G}(\mathcal{C})$ are the colors of the rims corresponding to the outputs, and the morphism $f$ in $\mathcal{Z}_{G}(\mathcal{C})$ is the color of the coupon $x$. For $m, n \geq 1$, set

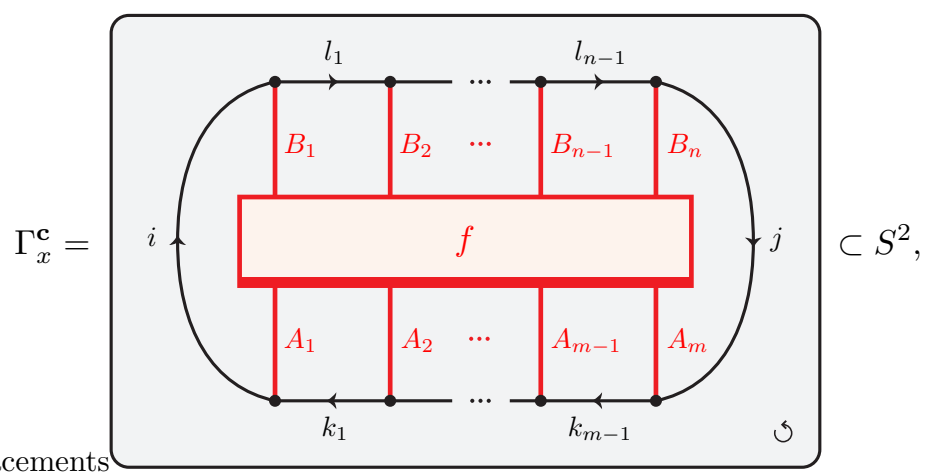

where the orientations (not shown in the picture) of the vertical arcs are induced by the orientations of the corresponding strands of $d$. For $m=0$ and $n \geq 1$, set

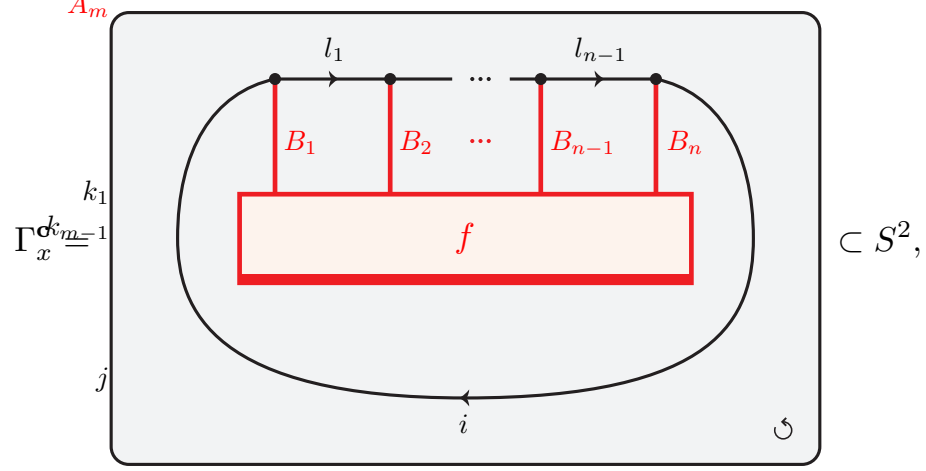

The case $m \geq 1$ and $n=0$ is similar. For $m=n=0$, set

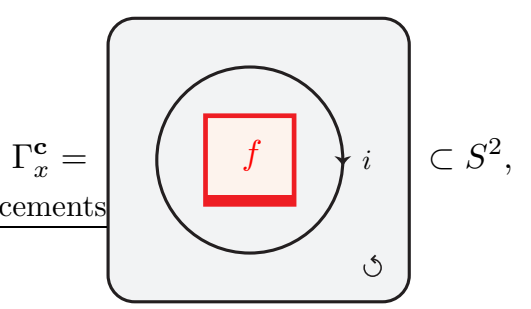

where here $f \in \operatorname{End}_{\mathcal{Z}_{G}(\mathcal{C})}\left(\mathbb{1}_{\mathcal{Z}_{G}(\mathcal{C})}\right)=\mathbb{k}$. 
(iv) Let $x$ be a crossing of $d$. A neighborhood of $x$ in $P$ looks as follows:

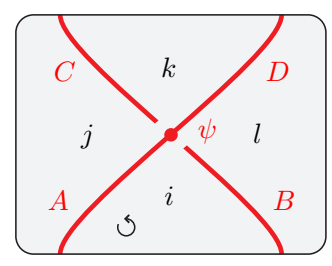

where objects $A, B, C, D \in \mathcal{Z}_{G}(\mathcal{C})$ are the colors of the rims adjacent to $x$, the isomorphism $\psi$ is the color of $x$, and $i, j, k, l \in I$ are the c-colors of the faces of $d$ adjacent to $x$. We associate with $x$ the knotted $\mathcal{C}$-net

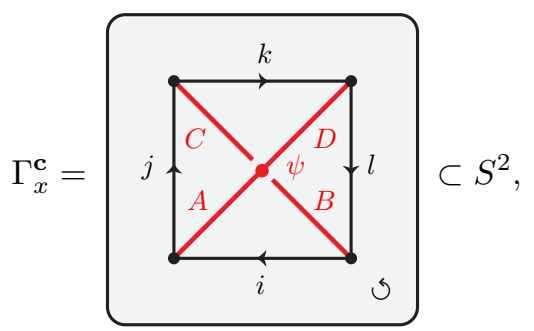

where the orientations of the diagonals (not shown in the picture) are induced by the orientations of the corresponding strands of $d$.

By Section 9.5, for any node $x$ of $d$, the knotted $\mathcal{C}$-net $\Gamma_{x}^{\mathbf{c}}$ yields a vector

$$
\mathbb{F}_{\mathcal{C}}\left(\Gamma_{x}^{\mathbf{c}}\right) \in \operatorname{Hom}_{\mathbb{k}}\left(H\left(\Gamma_{x}^{\mathbf{c}}\right), \operatorname{End}_{\mathcal{C}}(\mathbb{1})\right)=\operatorname{Hom}_{\mathbb{k}}\left(H\left(\Gamma_{x}^{\mathbf{c}}\right), \mathbb{k}\right)=H\left(\Gamma_{x}^{\mathbf{c}}\right)^{\star} .
$$

It results from the definitions that we have canonical isomorphisms

$$
H\left(\Gamma_{x}^{\mathbf{c}}\right) \simeq \bigotimes_{e_{x}} H_{\mathbf{c}}\left(e_{x}\right) \quad \text { and } \quad H\left(\Gamma_{x}^{\mathbf{c}}\right)^{\star} \simeq \bigotimes_{e_{x}} H_{\mathbf{c}}\left(e_{x}\right)^{\star}
$$

where $e_{x}$ runs over the rims of $d$ incident to $x$ and oriented away from $x$. The tensor product of the latter isomorphisms over all nodes $x$ of $d$ yields an isomorphism

$$
\bigotimes_{x} H\left(\Gamma_{x}^{\mathbf{c}}\right)^{\star} \simeq \bigotimes_{x} \bigotimes_{e_{x}} H_{\mathbf{c}}\left(e_{x}\right)^{\star} \simeq H_{\mathbf{c}}^{\star}
$$

The image under this isomorphism of the unordered tensor product $\bigotimes_{x} \mathbb{F}_{\mathcal{C}}\left(\Gamma_{x}\right)$ is a vector $V_{\mathbf{c}} \in H_{\mathbf{c}}^{\star}$. Set

$$
|\mathbf{c}|=V_{\mathbf{c}}\left(*_{\mathbf{c}}\right) \in \mathbb{k} \quad \text { and } \quad \operatorname{dim}(\mathbf{c})=\prod_{r \in \operatorname{Fac}(d)}(\operatorname{dim} \mathbf{c}(r))^{\chi(r)} \in \mathbb{k}
$$

where $\chi$ is the Euler characteristic. Finally, set

$$
|M, \Omega|_{\mathcal{C}}=\left(\operatorname{dim}\left(\mathcal{C}_{1}\right)\right)^{-|M \backslash P|} \sum_{\mathbf{c}} \operatorname{dim}(\mathbf{c})|\mathbf{c}| \in \mathbb{k},
$$

where c runs over all $G$-colorings of $d$ and the positive integer $|M \backslash P|$ is the number of $P$-balls (i.e., the number of connected components of $M \backslash P$ ).

Theorem 10.1. The scalar $|M, \Omega|_{\mathcal{C}}$ is a well-defined isomorphism invariant of the $\mathcal{Z}_{G}(\mathcal{C})$-colored $G$-graph $(M, \Omega)$. Also, $|M, \Omega|_{\mathcal{C}}$ is invariant under stabilization and conjugation of $(M, \Omega)$ (see Section 4.6 ).

The proof of Theorem 10.1 consists in verifying that the left-hand side of (11) remains invariant under the moves on knotted plexuses in skeletons (see the end of Section 10.1). This goes by combining the proofs of [TV2, Theorem 7.1] and [TV5, Theorem 15.7] and is left to the reader. The naturality of $\mathbb{F}_{\mathcal{C}}$ implies that $|M, \Omega|_{\mathcal{C}}$ is independent of the choice of the representative set $I$ of simple objects of $\mathcal{C}$. For 
$\Omega=\emptyset$, we obtain the invariant $|M, \emptyset|_{\mathcal{C}}=|M|_{\mathcal{C}}$ of the $G$-manifold $M$ defined in TV2, Theorem 7.1].

10.3. Proof of Theorem 8.1, The construction of the state sum graph TQFTs given in TV5, Section 15.7] applies (with colorings replaced by $G$-colorings) to the state sum invariant $|\cdot|_{\mathcal{C}}$ of $\mathcal{Z}_{G}(\mathcal{C})$-colored $G$-graphs (defined in Section 10.2) and produces a graph $\mathrm{HQFT}|\cdot|_{\mathcal{C}}$ over $\mathcal{Z}_{G}(\mathcal{C})$. It is clear from the definitions that this graph HQFT extends the state sum HQFT $|\cdot|_{\mathcal{C}}$. This proves Theorem 8.1 ,

10.4. Properties of $|\cdot|_{\mathcal{C}}$. We state two properties of the graph HQFT $|\cdot|_{\mathcal{C}}$. First, we compute $\left|S^{3}, \Omega\right|_{\mathcal{C}} \in \mathbb{k}$ for any $\mathcal{Z}_{G}(\mathcal{C})$-colored $G$-graph $\Omega$ in $S^{3}$. By TV4, the $G$-ribbon category $\mathcal{Z}_{G}(\mathcal{C})$ defines a monoidal functor $F_{\mathcal{Z}_{G}(\mathcal{C})}$ from the category of $\mathcal{Z}_{G}(\mathcal{C})$-colored $G$-graphs in $\mathbb{R}^{2} \times[0,1]$ to $\mathcal{Z}_{G}(\mathcal{C})$. In particular,

$$
F_{\mathcal{Z}_{G}(\mathcal{C})}(\Omega) \in \operatorname{End}_{\mathcal{Z}_{G}(\mathcal{C})}\left(\mathbb{1}_{\mathcal{Z}_{G}(\mathcal{C})}\right)=\mathbb{k} .
$$

In generalization of [TV5, Theorem 16.1] (where $G=1$ ), we claim that

$$
\left|S^{3}, \Omega\right|_{\mathcal{C}}=\left(\operatorname{dim}\left(\mathcal{C}_{1}\right)\right)^{-1} F_{\mathcal{Z}_{G}(\mathcal{C})}(\Omega) .
$$

The proof repeats the one of [V5, Theorem 16.1] with colorings replaced by $G$ colorings.

Second, we compute the isomorphism class of the module $|\Sigma|_{\mathcal{C}}$ for any connected $\mathcal{Z}_{G}(\mathcal{C})$-colored $G$-surface $\Sigma$ of genus $g \geq 0$. Let $\alpha_{1}, \beta_{1}, \ldots, \alpha_{g}, \beta_{g} \in G$ and $U_{\Sigma} \in$ $\mathcal{Z}_{G}(\mathcal{C})$ be as in Section 8.2. We claim that if the category $\mathcal{C}$ is additive, then

$$
|\Sigma|_{\mathcal{C}} \simeq \operatorname{Hom}_{\mathcal{Z}_{G}(\mathcal{C})}\left(\mathbb{1}_{\mathcal{Z}_{G}(\mathcal{C})}, \widetilde{C}_{\alpha_{1}, \beta_{1}} \otimes \cdots \otimes \widetilde{C}_{\alpha_{g}, \beta_{g}} \otimes U_{\Sigma}\right),
$$

where for $\alpha, \beta \in G$, the object $\widetilde{C}_{\alpha, \beta}=\left(C_{\alpha, \beta}, \sigma^{\alpha, \beta}\right)$ of $\mathcal{Z}_{G}(\mathcal{C})$ is defined by

$$
C_{\alpha, \beta}=\bigoplus_{i \in I_{\alpha}, j \in I_{\beta}} i^{*} \otimes j^{*} \otimes i \otimes j
$$

and, for any $X \in \mathcal{C}_{1}$,

$$
\sigma_{X}^{\alpha, \beta}=\sum_{\substack{i, k \in I_{\alpha} \\ j, l \in I_{\beta} \\ z \in I_{1}}}^{\downarrow_{f}} \underbrace{\uparrow_{j}}_{\uparrow_{i}}
$$

Here, complementary curvilinear boxes of the same color represent the projection/inclusion determined by an isotypic subobject, see [TV5, Section 4.6] for details. Formula (13) generalizes [TV5, Theorem 16.2] where $G=1$. The proof repeats the one given there with colorings replaced by $G$-colorings.

\section{Computations in GRAPH HQFTs}

In this section, $Z: \operatorname{Cob}_{\mathcal{B}}^{G} \rightarrow \operatorname{Mod}_{\mathrm{k}}$ is an arbitrary graph HQFT over a $G$-crossed category $\mathcal{B}$ over $\mathbb{k}$. We give a surgery formula for $Z$ using so-called torus vectors.

11.1. Graphs without free ends. A $\mathcal{B}$-colored $G$-graph $(M, \Omega)$ has no free ends if $\Omega \cap \partial M=\emptyset$, that is, if the endpoints of all arcs of $\Omega$ lie on the bases of the coupons. Such a graph represents a morphism $(M, \Omega)_{+}: \emptyset \rightarrow \partial M$ and a morphism $(M, \Omega)_{-}:-\partial M \rightarrow \emptyset$ in the category $\operatorname{Cob}_{\mathcal{B}}^{G}$. Recall the $\mathbb{k}$-linear isomorphism $Z_{0}: \mathbb{k} \simeq Z(\emptyset)$ and set

$$
Z_{+}(M, \Omega)=Z\left((M, \Omega)_{+}\right) Z_{0}: \mathbb{k} \rightarrow Z(\partial M)
$$


and

$$
Z_{-}(M, \Omega)=Z_{0}^{-1} Z\left((M, \Omega)_{-}\right): Z(-\partial M) \rightarrow \mathbb{k} .
$$

If $\partial M=\emptyset$, then any $\mathcal{B}$-colored $G$-graph $\Omega \subset M$ has no free ends and $(M, \Omega)_{+}=$ $(M, \Omega)_{-}$. In this case, the $\mathbb{k}$-linear homomorphism

$$
Z_{0}^{-1} Z_{+}(M, \Omega)=Z_{-}(M, \Omega) Z_{0}: \mathbb{k} \rightarrow \mathbb{k}
$$

is multiplication by the scalar $Z(M, \Omega) \in \mathbb{k}$ defined in Section 8.1 .

11.2. Torus vectors. We endow the unit disk $D^{2}=\{z \in \mathbb{C},|z| \leq 1\}$ and the unit circle $S^{1}=\partial D^{2}$ with the counterclockwise orientation. Endow the torus $S^{1} \times S^{1}$ with the product orientation and the base point $*=(1,1)$. For each $\alpha \in G=$ $\pi_{1}(\mathbf{X}, \mathbf{x})$, we let $g_{\alpha}$ be the unique homotopy class of maps $\left(S^{1} \times S^{1}, *\right) \rightarrow(\mathbf{X}, \mathbf{x})$ which carry the loops

$$
t \in[0,1] \mapsto\left(e^{2 \pi i t}, 1\right) \in S^{1} \times S^{1} \quad \text { and } \quad t \in[0,1] \mapsto\left(1, e^{2 \pi i t}\right) \in S^{1} \times S^{1}
$$

into loops in $(\mathbf{X}, \mathbf{x})$ representing respectively $\alpha$ and $1 \in G$. Then $\mathbb{T}_{\alpha}=\left(S^{1} \times S^{1}, g_{\alpha}\right)$ is a $\mathcal{B}$-colored $G$-surface with an empty set of marked points. Let $V=-\left(S^{1} \times D^{2}\right)$ be the solid torus with orientation opposite to the product orientation and with boundary $\partial V=S^{1} \times S^{1}$ pointed by $(\partial V)_{\bullet}=\{*\}$. The homotopy class of maps $g_{\alpha}$ above extends uniquely to a homotopy class of maps $\widetilde{g}_{\alpha}:\left(V,(\partial V)_{\bullet}\right) \rightarrow(\mathbf{X}, \mathbf{x})$. The triple $V_{\alpha}=\left(V, \emptyset, \widetilde{g}_{\alpha}\right)$ is a $\mathcal{B}$-colored $G$-graph with no free ends and with $\partial\left(V_{\alpha}\right)=\mathbb{T}_{\alpha}$. The $\alpha$-torus vector of $Z$ is the vector $Z_{+}\left(V_{\alpha}\right)\left(1_{\mathbb{k}}\right) \in Z\left(\mathbb{T}_{\alpha}\right)$.

11.3. The surgery formula. Let $(M, \Omega)$ be a $\mathcal{B}$-colored $G$-graph where $M$ is a closed connected oriented 3-manifold. We give a surgery formula for $Z(M, \Omega) \in \mathbb{k}$. To this end, present $M$ as the result of surgery on $S^{3}=\mathbb{R}^{3} \cup\{\infty\}$ along a framed link $L=L_{1} \cup \cdots \cup L_{n} \subset \mathbb{R}^{2} \times(0,1)$ with $n \geq 1$ components. Pick a closed regular neighborhood $U \subset S^{3}$ of $L$ and let $E=S^{3} \backslash \operatorname{Int}(U)$ be the exterior of $L$ in $S^{3}$. We endow both $U$ and $E$ with orientation induced by the right-handed orientation of $S^{3}$. By the definition of surgery, $M$ is obtained by gluing $n$ solid tori to $E$. Deforming if necessary $\Omega$ in $M$, we assume that $\Omega \subset \operatorname{Int}(E) \subset M$ and that $\Omega_{\bullet} \subset \partial E$ when $\Omega \neq \emptyset$.

We now turn the pair $(E, \Omega)$ into a $\mathcal{B}$-colored $G$-graph. Let $n\left(S^{1} \times S^{1}\right)$ be a disjoint union of $n$ copies of $S^{1} \times S^{1}$. Orient the link $L$ arbitrarily and pick an orientation-preserving diffeomorphism

$$
f_{L}: n\left(S^{1} \times S^{1}\right) \longrightarrow \partial U=-\partial E
$$

which, for all $p \in S^{1}$ and $q=1, \ldots, n$, carries the $q$-th copy of $S^{1} \times\{p\}$ to a positively oriented meridian of $L_{q}$ in $\partial U$ and carries the $q$-th copy of $\{p\} \times S^{1}$ to the positively oriented longitude of $L_{q}$ in $\partial U$ determined by the framing of $L$. Let $(\partial E)$. $\subset \partial E$ be the set consisting of the images under $f_{L}$ of the base points $*=(1,1)$ of the $n$ copies of $S^{1} \times S^{1}$. We choose $f_{L}$ so that $\Omega_{\bullet} \subset(\partial E)$. when $\Omega \neq \emptyset$. So, $E$ becomes an oriented 3-manifold with pointed boundary and $\Omega$ is a ribbon graph in $E$ with no free ends. Pick in the given homotopy class of maps $\left(M \backslash \Omega, \Omega_{\bullet}\right) \rightarrow(\mathbf{X}, \mathbf{x})$ a map carrying $(\partial E) \bullet \subset M \backslash \Omega$ to $\mathbf{x}$ and restrict it to $E \backslash \Omega$. This gives a map $g:\left(E \backslash \Omega,(\partial E)_{\bullet}\right) \rightarrow(\mathbf{X}, \mathbf{x})$ turning $(E, \Omega)$ into a $G$-graph. The $\mathcal{B}$-coloring of $(M, \Omega)$ induces a $\mathcal{B}$-coloring of $(E, \Omega)$. Indeed, when $\Omega \neq \emptyset$, pick a 1 -system and a 2 -system of tracks for $\Omega$ in $E$ that start in the point of $\Omega_{\bullet} \subset(\partial E)$. These systems together with their evaluation under the $\mathcal{B}$-coloring of $(M, \Omega)$ define a $\mathcal{B}$-coloring of $(E, \Omega)$ as in Section [5.3. Thus, $(E, \Omega)$ is a $\mathcal{B}$-colored $G$-graph with no free ends.

The homotopy class of the map $g f_{L}: n\left(S^{1} \times S^{1}\right) \rightarrow(\mathbf{X}, \mathbf{x})$ turns $n\left(S^{1} \times S^{1}\right)$ into a $G$-surface which, in the notation of Section 11.2 is a disjoint union $\sqcup_{q=1}^{n} \mathbb{T}_{\alpha_{q}}$ where $\alpha_{q} \in G=\pi_{1}(\mathbf{X}, \mathbf{x})$ is represented by the image under $g f_{L}$ of the loop 
$S^{1} \rightarrow n\left(S^{1} \times S^{1}\right)$ carrying any $s \in S^{1}$ to the point $(s, 1)$ in the $q$-th copy of $S^{1} \times S^{1}$. Then the map $f_{L}$ above is an isomorphism of $G$-surfaces $\sqcup_{q=1}^{n} \mathbb{T}_{\alpha_{q}} \rightarrow-\partial E$. Its cylinder (see Section 7.3 ) induces a $\mathbb{k}$-linear isomorphism

$$
Z\left(f_{L}\right)=Z\left(\operatorname{cyl}\left(f_{L}\right)\right): Z\left(\sqcup_{q=1}^{n} \mathbb{T}_{\alpha_{q}}\right) \rightarrow Z(-\partial E) .
$$

The monoidal constraints of the graph HQFT $Z$ induce a $\mathbb{k}$-linear isomorphism

$$
\xi_{n}: Z\left(\mathbb{T}_{\alpha_{1}}\right) \otimes \cdots \otimes Z\left(\mathbb{T}_{\alpha_{n}}\right) \rightarrow Z\left(\sqcup_{q=1}^{n} \mathbb{T}_{\alpha_{q}}\right) .
$$

Composing these isomorphisms with the homomorphism $Z_{-}(E, \Omega): Z(-\partial E) \rightarrow \mathbb{k}$ (see Section 11.1), we obtain a $\mathbb{k}$-linear homomorphism

$$
Z^{L}=Z_{-}(E, \Omega) \circ Z\left(f_{L}\right) \circ \xi_{n}: Z\left(\mathbb{T}_{\alpha_{1}}\right) \otimes \cdots \otimes Z\left(\mathbb{T}_{\alpha_{n}}\right) \rightarrow \mathbb{k} .
$$

Lemma 11.1. For any $\alpha \in G$, let $w_{\alpha} \in Z\left(\mathbb{T}_{\alpha}\right)$ be the $\alpha$-torus vector of $Z$. Then

$$
Z(M, \Omega)=Z^{L}\left(w_{\alpha_{1}} \otimes \cdots \otimes w_{\alpha_{n}}\right) .
$$

Proof. Recall from Section 11.2 the $\mathcal{B}$-colored $G$-graphs $\left\{V_{\alpha}\right\}_{\alpha \in G}$ with $\partial V_{\alpha}=\mathbb{T}_{\alpha}$. Since $(M, \Omega)$ is obtained by gluing $V_{\alpha_{1}} \sqcup \cdots \sqcup V_{\alpha_{n}}$ to $(E, \Omega)$ along $f_{L}$, we have

$$
(M, \Omega)_{+}=(E, \Omega)_{-} \circ \operatorname{cyl}\left(f_{L}\right) \circ\left(\left(V_{\alpha_{1}}\right)_{+} \sqcup \cdots \sqcup\left(V_{\alpha_{n}}\right)_{+}\right): \emptyset \rightarrow \emptyset
$$

in the category $\operatorname{Cob}_{\mathcal{B}}^{G}$. Applying the functor $Z$, we get

$$
Z\left((M, \Omega)_{+}\right)=Z\left((E, \Omega)_{-}\right) \circ Z\left(f_{L}\right) \circ Z\left(\left(V_{\alpha_{1}}\right)_{+} \sqcup \cdots \sqcup\left(V_{\alpha_{n}}\right)_{+}\right): Z(\emptyset) \rightarrow Z(\emptyset) .
$$

Since $Z(M, \Omega)=Z_{0}^{-1} Z\left((M, \Omega)_{+}\right) Z_{0}\left(1_{\mathbb{k}}\right)$, we have

$$
Z(M, \Omega)=Z_{0}^{-1} Z\left((E, \Omega)_{-}\right) Z\left(f_{L}\right) Z\left(\left(V_{\alpha_{1}}\right)_{+} \sqcup \cdots \sqcup\left(V_{\alpha_{n}}\right)_{+}\right) Z_{0}\left(1_{\mathbb{k}}\right) .
$$

Now, by definition, $Z_{-}(E, \Omega)=Z_{0}^{-1} Z\left((E, \Omega)_{-}\right)$. Also, the monoidality of $Z$ and the definition of the torus vectors imply that

$$
Z\left(\left(V_{\alpha_{1}}\right)_{+} \sqcup \cdots \sqcup\left(V_{\alpha_{n}}\right)_{+}\right) Z_{0}\left(1_{\mathbb{k}}\right)=\xi_{n}\left(w_{\alpha_{1}} \otimes \cdots \otimes w_{\alpha_{n}}\right) .
$$

Therefore,

$$
Z(M, \Omega)=Z_{-}(E, \Omega) Z\left(f_{L}\right) \xi_{n}\left(w_{\alpha_{1}} \otimes \cdots \otimes w_{\alpha_{n}}\right)=Z^{L}\left(w_{\alpha_{1}} \otimes \cdots \otimes w_{\alpha_{n}}\right) .
$$

We now evaluate $Z^{L}$ on certain vectors. Consider the solid torus $W=D^{2} \times S^{1}$ with product orientation and base point $*=(1,1)$ in $\partial W=S^{1} \times S^{1}$. Consider the knot $K=\{0\} \times S^{1} \subset \operatorname{Int}(W)$ with orientation induced by the opposite (clockwise) orientation of $S^{1}$ and with framing $((1,0), 0)$ at all points. Insert in $K$ a coupon transversal to the framing and having one input and one output (see Section 4.6). We choose the bottom base of the coupon so that the input is directed out of the coupon. In this way, we stabilize $K$ into a ribbon graph $K^{s} \subset W$. For $\alpha \in G$, the homotopy class of maps $g_{\alpha}:\left(S^{1} \times S^{1}, *\right) \rightarrow(\mathbf{X}, \mathbf{x})$ from Section 11.2 extends uniquely to a homotopy class of maps

$$
g_{\alpha}^{+}:\left(W \backslash K^{s},(\partial W) \bullet=\{*\}\right) \rightarrow(\mathbf{X}, \mathbf{x}) .
$$

The triple $W_{\alpha}=\left(W, K^{s}, g_{\alpha}^{+}\right)$is a $G$-graph with no free ends. Note that for any track $\gamma$ of a stratum of $K^{s}$, the associated element $\mu_{\gamma} \in \pi_{1}\left(W \backslash K^{s}, *\right)$ is carried by $g_{\alpha}^{+}$to $\alpha \in \pi_{1}(\mathbf{X}, \mathbf{x})=G$. Each object $X \in \mathcal{B}_{\alpha}$ determines a $\mathcal{B}$-coloring of $W_{\alpha}$ as follows. Pick a track of the only arc and a track of the only coupon of $K^{s}$. These tracks forms a 1-system and a 2-system of tracks for $W_{\alpha}$ and so, when colored by the object $X$ and the morphism $\operatorname{id}_{\varphi_{1}(X)}$, they determine a $\mathcal{B}$-coloring of $W_{\alpha}$ (see Section 5.3). This yields a $\mathcal{B}$-colored $G$-graph $W_{\alpha}^{X}$ with no free ends such that $\partial\left(W_{\alpha}^{X}\right)=\mathbb{T}_{\alpha}$. Set

$$
[X]=Z_{+}\left(W_{\alpha}^{X}\right)\left(1_{\mathbb{k}}\right) \in Z\left(\mathbb{T}_{\alpha}\right) .
$$

For any $X_{1} \in \mathcal{B}_{\alpha_{1}}, \ldots, X_{n} \in \mathcal{B}_{\alpha_{n}}$, gluing $W_{\alpha_{1}}^{X_{1}} \sqcup \cdots \sqcup W_{\alpha_{n}}^{X_{n}}$ to $(E, \Omega)$ along $f_{L}$ yields a $\mathcal{B}$-colored $G$-graph $\left(S^{3}, T_{X_{1}, \ldots, X_{n}}\right)$. Note that the underlying ribbon graph 
of $T_{X_{1}, \ldots, X_{n}} \subset S^{3}$ is the union $L^{s} \cup \Omega$, where $L^{s}$ is obtained from $L$ by stabilizing each of its components.

Lemma 11.2. For any $X_{1} \in \mathcal{B}_{\alpha_{1}}, \ldots, X_{n} \in \mathcal{B}_{\alpha_{n}}$,

$$
Z^{L}\left(\left[X_{1}\right] \otimes \cdots \otimes\left[X_{n}\right]\right)=Z\left(S^{3}, T_{X_{1}, \ldots, X_{n}}\right) \in \mathbb{k} .
$$

Proof. The monoidality of the functor $Z$ implies that

$$
\xi_{n}\left(\left[X_{1}\right] \otimes \cdots \otimes\left[X_{n}\right]\right)=Z_{+}\left(W_{\alpha_{1}}^{X_{1}} \sqcup \cdots \sqcup W_{\alpha_{n}}^{X_{n}}\right)\left(1_{\mathbb{k}}\right) .
$$

Then

$$
\begin{gathered}
Z^{L}\left(\left[X_{1}\right] \otimes \cdots \otimes\left[X_{n}\right]\right)=Z_{-}(E, \Omega) Z\left(f_{L}\right) Z_{+}\left(W_{\alpha_{1}}^{X_{1}} \sqcup \cdots \sqcup W_{\alpha_{n}}^{X_{n}}\right)\left(1_{\mathbb{k}}\right) \\
=Z_{0}^{-1} Z\left((E, \Omega)_{-}\right) Z\left(f_{L}\right) Z\left(\left(W_{\alpha_{1}}^{X_{1}} \sqcup \cdots \sqcup W_{\alpha_{n}}^{X_{n}}\right)_{+}\right) Z_{0}\left(1_{\mathbb{k}}\right)=Z\left(S^{3}, T_{X_{1}, \ldots, X_{n}}\right),
\end{gathered}
$$

where the last equality follows from the functoriality of $Z$.

\section{Proof of Theorem 8.2}

In this section, we prove Theorem 8.2 Recall that $\mathbb{k}$ is an algebraically closed field and $\mathcal{C}=\oplus_{\alpha \in G} \mathcal{C}_{\alpha}$ is an additive spherical $G$-fusion category over $\mathbb{k}$ such that $\operatorname{dim}\left(\mathcal{C}_{1}\right) \neq 0$. By Section $2.8, \mathcal{Z}_{G}(\mathcal{C})$ is an additive anomaly free $G$-modular category whose canonical rank is equal to $\operatorname{dim}\left(\mathcal{C}_{1}\right)$. Recall that $I=\amalg_{\alpha \in G} I_{\alpha}$ denotes a representative set of simple objects of $\mathcal{C}$. Let $\mathcal{J}=\amalg_{\alpha \in G} \mathcal{J}_{\alpha}$ be a representative set of simple objects of $\mathcal{Z}_{G}(\mathcal{C})$.

The surgery graph HQFT $\tau_{\mathcal{Z}_{G}(\mathcal{C})}$ and the state sum graph HQFT $|\cdot|_{\mathcal{C}}$ are (symmetric) monoidal functors $\operatorname{Cob}_{\mathcal{Z}_{G}(\mathcal{C})}^{G} \rightarrow \operatorname{Mod}_{\mathbb{k}}$. Now the category $\operatorname{Cob}_{\mathcal{Z}_{G}(\mathcal{C})}^{G}$ is left rigid (see Section 7.3 ) and $\tau_{\mathcal{Z}_{G}(\mathcal{C})}$ is non-degenerate (see Section 8.2 ). Thus, by TV5, Lemma 17.2], we only need to prove that:

(a) for any $\mathcal{Z}_{G}(\mathcal{C})$-colored $G$-surface $\Sigma$,

$$
|\Sigma|_{\mathcal{C}} \simeq \tau_{\mathcal{Z}_{G}(\mathcal{C})}(\Sigma)
$$

(b) for any $\mathcal{Z}_{G}(\mathcal{C})$-colored $G$-graph $\Omega$ in a closed oriented 3-manifold $M$,

$$
|M, \Omega|_{\mathcal{C}}=\tau_{\mathcal{Z}_{G}(\mathcal{C})}(M, \Omega) \text {. }
$$

12.1. Proof of (a). For $\alpha, \beta \in G$, recall the object $\widetilde{C}_{\alpha, \beta} \in \mathcal{Z}_{G}(\mathcal{C})$ from Section 10.4 . By [TV3, Theorem A1], this object is the coend

$$
\widetilde{C}_{\alpha, \beta}=\int^{X \in \mathcal{Z}_{\beta}(\mathcal{C})}\left(\varphi_{\alpha}(X)\right)^{*} \otimes X .
$$

Now, since $\mathcal{Z}_{\beta}(\mathcal{C})$ is additive and finitely semisimple with $\mathcal{J}_{\beta}$ as a representative set of simple objects, we have:

$$
\int^{X \in \mathcal{Z}_{\beta}(\mathcal{C})}\left(\varphi_{\alpha}(X)\right)^{*} \otimes X=\bigoplus_{J \in \mathcal{J}_{\beta}}\left(\varphi_{\alpha}(J)\right)^{*} \otimes J .
$$

Consequently, the uniqueness of a coend implies that

$$
\widetilde{C}_{\alpha, \beta} \simeq \bigoplus_{J \in \mathcal{J}_{\beta}}\left(\varphi_{\alpha}(J)\right)^{*} \otimes J .
$$

Let $\Sigma$ be a connected $\mathcal{Z}_{G}(\mathcal{C})$-colored $G$-surface of genus $g \geq 0$. The surface $\Sigma$ carries a base point $*$, a finite set of marked points $A=\left\{a_{1}, \ldots, a_{m}\right\}$, and a homotopy class of maps $(\Sigma \backslash A, *) \rightarrow(\mathbf{X}, \mathbf{x})$. Pick a track $\gamma_{i}$ of $a_{i}$ for each $i \in$ $\{1, \ldots m\}$. Recall from Section 6.1 the homotopy class $\mu_{\gamma_{i}} \in \pi_{1}(\Sigma \backslash A, *)$ of the loop encircling $a_{i}$. The group $\pi_{1}(\Sigma \backslash A, *)$ is generated by $\mu_{\gamma_{1}}, \ldots, \mu_{\gamma_{m}}$ and $2 g$ elements $\alpha_{1}, \beta_{1}, \ldots, \alpha_{g}, \beta_{g}$ subject to the only relation

$$
\left[\alpha_{1}, \beta_{1}\right] \cdots\left[\alpha_{g}, \beta_{g}\right]\left(\mu_{\gamma_{1}}\right)^{\varepsilon_{1}} \cdots\left(\mu_{\gamma_{m}}\right)^{\varepsilon_{m}}=1,
$$


where $[\alpha, \beta]=\alpha^{-1} \beta^{-1} \alpha \beta$ and $\varepsilon_{i}= \pm 1$ is the sign of $a_{i}$. Consider the object $U_{\Sigma} \in \mathcal{Z}_{G}(\mathcal{C})$ defined as in Section 8.2. By (13), we have

$$
|\Sigma|_{\mathcal{C}} \simeq \operatorname{Hom}_{\mathcal{Z}_{G}(\mathcal{C})}\left(\mathbb{1}_{\mathcal{Z}_{G}(\mathcal{C})}, \widetilde{C}_{\alpha_{1}, \beta_{1}} \otimes \cdots \otimes \widetilde{C}_{\alpha_{g}, \beta_{g}} \otimes U_{\Sigma}\right)
$$

Now (15) implies that

$$
\widetilde{C}_{\alpha_{1}, \beta_{1}} \otimes \cdots \otimes \widetilde{C}_{\alpha_{g}, \beta_{g}} \simeq \bigoplus_{J_{1} \in \mathcal{I}_{\beta_{1}}, \ldots, J_{g} \in \mathcal{I}_{\beta_{g}}}\left(\varphi_{\alpha_{1}}\left(J_{1}\right)^{*} \otimes J_{1}\right) \otimes \cdots \otimes\left(\varphi_{\alpha_{g}}\left(J_{g}\right)^{*} \otimes J_{g}\right) .
$$

The last two formulas together with the additivity of $\mathcal{Z}_{G}(\mathcal{C})$ imply that $|\Sigma|_{\mathcal{C}}$ is isomorphic to

$$
\bigoplus_{J_{1} \in \mathcal{I}_{\beta_{1}}, \ldots, J_{g} \in \mathcal{I}_{\beta g}} \operatorname{Hom}_{\mathcal{Z}_{G}(\mathcal{C})}\left(\mathbb{1}_{\mathcal{Z}_{G}(\mathcal{C})},\left(\varphi_{\alpha_{1}}\left(J_{1}\right)^{*} \otimes J_{1}\right) \otimes \cdots \otimes\left(\varphi_{\alpha_{g}}\left(J_{g}\right)^{*} \otimes J_{g}\right) \otimes U_{\Sigma}\right) .
$$

Now (8) applied with $\mathcal{B}=\mathcal{Z}_{G}(\mathcal{C})$ implies that the latter vector space is isomorphic to $\tau_{\mathcal{Z}_{G}(\mathcal{C})}(\Sigma)$. Consequently $|\Sigma|_{\mathcal{C}}$ is isomorphic to $\tau_{\mathcal{Z}_{G}(\mathcal{C})}(\Sigma)$.

The case of disconnected $\Sigma$ is deduced from the case of connected $\Sigma$ using Formula (7) applied to the graph HQFTs $|\cdot|_{\mathcal{C}}$ and $\tau_{\mathcal{Z}_{G}(\mathcal{C})}$.

12.2. Proof of (b). Let $\alpha \in G$. Recall that any object $X \in \mathcal{Z}_{\alpha}(\mathcal{C})$ determines a vector $[X] \in\left|\mathbb{T}_{\alpha}\right|_{\mathcal{C}}$ as in (14) with $\mathcal{B}=\mathcal{Z}_{G}(\mathcal{C})$. Proceeding as in TV5, Section 17.4], we obtain that the family $\{[J]\}_{J \in \mathcal{J}_{\alpha}}$ is a basis of $\left|\mathbb{T}_{\alpha}\right|_{\mathcal{C}}$ and that the $\alpha$-torus vector $w_{\alpha} \in\left|\mathbb{T}_{\alpha}\right|_{\mathcal{C}}$ of $|\cdot|_{\mathcal{C}}$ is computed in this basis by

$$
w_{\alpha}=\left(\operatorname{dim}\left(\mathcal{C}_{1}\right)\right)^{-1} \sum_{J \in \mathcal{J}_{\alpha}} \operatorname{dim}(J)[J] .
$$

Pick a $\mathcal{Z}_{G}(\mathcal{C})$-colored $G$-graph $\Omega$ in a closed oriented 3-manifold $M$. Since $|M, \Omega|_{\mathcal{C}}$ and $\tau_{\mathcal{Z}_{G}(\mathcal{C})}(M, \Omega)$ are multiplicative under disjoint union, it suffices to consider the case where $M$ is connected. Present $M$ by surgery on $S^{3}$ along a framed oriented link $L=L_{1} \cup \cdots \cup L_{n} \subset S^{3}$. We use the notation of Section 11.3 with $Z=|\cdot|_{\mathcal{C}}$. By Lemma 11.1 and Formula (16),

$$
\begin{gathered}
|M, \Omega|_{\mathcal{C}}=Z^{L}\left(w_{\alpha_{1}} \otimes \cdots \otimes w_{\alpha_{n}}\right) \\
=\sum_{J_{1} \in \mathcal{J}_{\alpha_{1}}, \ldots, J_{n} \in \mathcal{J}_{\alpha_{n}}}\left(\prod_{q=1}^{n} \frac{\operatorname{dim}\left(J_{q}\right)}{\operatorname{dim}\left(\mathcal{C}_{1}\right)}\right) Z^{L}\left(\left[J_{1}\right] \otimes \cdots \otimes\left[J_{n}\right]\right) .
\end{gathered}
$$

Using the notation of Section 11.3, it follows from Lemma 11.2 and Formula (12) that for any $J_{1} \in \mathcal{J}_{\alpha_{1}}, \ldots, J_{n} \in \mathcal{J}_{\alpha_{n}}$,

$$
\left.Z^{L}\left(\left[J_{1}\right] \otimes \cdots \otimes\left[J_{n}\right]\right)\right)=\left|S^{3}, T_{J_{1}, \ldots, J_{n}}\right|_{\mathcal{C}}=\left(\operatorname{dim}\left(\mathcal{C}_{1}\right)\right)^{-1} F_{\mathcal{Z}_{G}(\mathcal{C})}\left(T_{J_{1}, \ldots, J_{n}}\right) .
$$

Therefore

$$
\begin{gathered}
|M, \Omega|_{\mathcal{C}}=\sum_{J_{1} \in \mathcal{J}_{\alpha_{1}}, \ldots, J_{n} \in \mathcal{J}_{\alpha_{n}}}\left(\prod_{q=1}^{n} \frac{\operatorname{dim}\left(J_{q}\right)}{\operatorname{dim}\left(\mathcal{C}_{1}\right)}\right)\left(\operatorname{dim}\left(\mathcal{C}_{1}\right)\right)^{-1} F_{\mathcal{Z}_{G}(\mathcal{C})}\left(T_{J_{1}, \ldots, J_{n}}\right) \\
=\left(\operatorname{dim}\left(\mathcal{C}_{1}\right)\right)^{-n-1} \sum_{J_{1} \in \mathcal{J}_{\alpha_{1}}, \ldots, J_{n} \in \mathcal{J}_{\alpha_{n}}}\left(\prod_{q=1}^{n} \operatorname{dim}\left(J_{q}\right)\right) F_{\mathcal{Z}_{G}(\mathcal{C})}\left(T_{J_{1}, \ldots, J_{n}}\right)=\tau_{\mathcal{Z}_{G}(\mathcal{C})}(M, \Omega),
\end{gathered}
$$

where the last equality is the definition of $\tau_{\mathcal{Z}_{G}(\mathcal{C})}(M, \Omega)$, see Formula (9).

\section{Appendix A. The Crossing and Braiding of the graded Center}

In this appendix, we summarize the construction of the canonical crossing and braiding of the $G$-center of a $G$-graded category, referring to [TV3] for details. We formulate these constructions for the class of non-singular $G$-graded categories over a commutative ring which includes the class of $G$-fusion categories over a field. 
A.1. Non-singular graded categories. A monoidal category is pure if $f \otimes \operatorname{id}{ }_{X}=$ $\operatorname{id}_{X} \otimes f$ for all object $X$ and all endomorphism $f$ of the monoidal unit $\mathbb{1}$. In a pure pivotal category, the left and right traces of endormorphisms are $\otimes$-multiplicative. By [TV5, Remarks 4.2.2], a k-linear monoidal category with simple monoidal unit is pure. Note also that the $G$-center of a pure $G$-graded category is pure.

An idempotent in a category is an endomorphism $e$ of an object such that $e^{2}=e$. An idempotent $e: X \rightarrow X$ splits if there is an object $E$ and morphisms $p: X \rightarrow E$ and $q: E \rightarrow X$ such that $q p=e$ and $p q=\mathrm{id}_{E}$. Note that such a splitting triple $(E, p, q)$ of $e$ is unique up to isomorphism. A category with split idempotents is a category in which all idempotents split.

Following [TV3, a $G$-graded category $\mathcal{C}$ is non-singular if it is pure, has split idempotents, and for all $\alpha \in G$, the subcategory $\mathcal{C}_{\alpha}$ of $\mathcal{C}$ has at least one object whose left dimension is invertible in $\operatorname{End}_{\mathcal{C}}(\mathbb{1})$.

Any $G$-fusion category $\mathcal{C}$ over $\mathbb{k}$ is pure (since its monoidal unit $\mathbb{1}$ is simple) and both the left and right dimensions of simple objects of $\mathcal{C}$ are invertible in $\operatorname{End}_{\mathcal{C}}(\mathbb{1})=\mathbb{k}$ (see [TV5, Section 4.4.2]). Also, if $\mathbb{k}$ is a field, then $\mathcal{C}$ has split idempotents. Therefore any $G$-fusion category over a field is non-singular.

A.2. Notation. We fix until the end of the appendix a non-singular $G$-graded category $\mathcal{C}$ over $\mathbb{k}$. Denote by $\mathcal{E}$ the class of homogeneous objects of $\mathcal{C}$ with invertible left dimension. This class decomposes as $\mathcal{E}=\amalg_{\alpha \in G} \mathcal{E}_{\alpha}$, where $\mathcal{E}_{\alpha}=\mathcal{E} \cap \mathcal{C}_{\alpha}$. (Note that $\mathcal{E}_{\alpha} \neq \emptyset$ since $\mathcal{C}$ is non-singular.) For $V \in \mathcal{E}$, we set

$$
d_{V}=\operatorname{dim}_{l}(V) \in \operatorname{End}_{\mathcal{C}}(\mathbb{1}) .
$$

By [TV3, Theorem 4.1], the $G$-center $\mathcal{Z}_{G}(\mathcal{C})$ of $\mathcal{C}$ has a canonical structure of a $G$-braided category. In the next sections, we recall the construction of the crossing and of the (enhanced) $G$-braiding of $\mathcal{Z}_{G}(\mathcal{C})$. We also compute the twist of $\mathcal{Z}_{G}(\mathcal{C})$.

A.3. The crossing. The crossing in $\mathcal{Z}_{G}(\mathcal{C})$ is constructed in three steps. At Step 1, we associate a monoidal endofunctor of $\mathcal{Z}_{G}(\mathcal{C})$ to each homogeneous object of $\mathcal{C}$ with invertible left dimension. At Step 2, we construct a system of isomorphisms between these endofunctors. At Step 3, we define the crossing as the limit of the resulting projective system of endofunctors and isomorphisms.

Step 1. For any $V \in \mathcal{E}$, we define a monoidal endofunctor $\varphi_{V}$ of $\mathcal{Z}_{G}(\mathcal{C})$ as follows. For any $(A, \sigma) \in \mathcal{Z}_{G}(\mathcal{C})$, the morphism

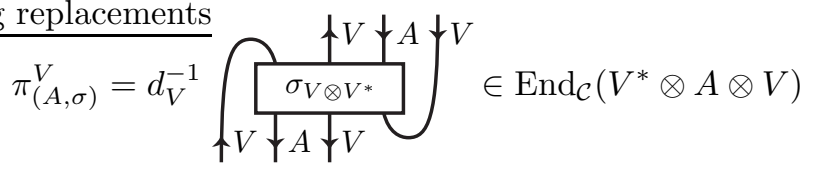

is an idempotent. Since all idempotents in $\mathcal{C}$ split, there exist an object $E_{(A, \sigma)}^{V} \in \mathcal{C}$ and morphisms $p_{(A, \sigma)}^{V}: V^{*} \otimes A \otimes V \rightarrow E_{(A, \sigma)}^{V}$ and $q_{(A, \sigma)}^{V}: E_{(A, \sigma)}^{V} \rightarrow V^{*} \otimes A \otimes V$ such that

$$
\pi_{(A, \sigma)}^{V}=q_{(A, \sigma)}^{V} p_{(A, \sigma)}^{V} \quad \text { and } \quad p_{(A, \sigma)}^{V} q_{(A, \sigma)}^{V}=\operatorname{id}_{E_{(A, \sigma)}^{V}} .
$$

When $A$ is homogeneous, we can and always choose $E_{(A, \sigma)}^{V}$ to be homogeneous (of degree $\left.|V|^{-1}|A||V|\right)$. We will depict the morphisms $p_{(A, \sigma)}^{V}$ and $q_{(A, \sigma)}^{V}$ as

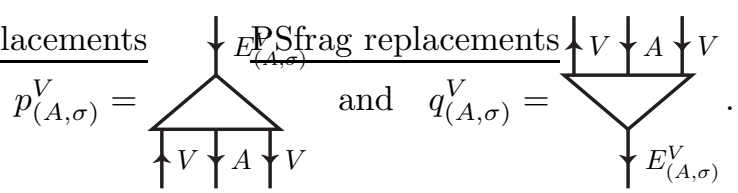


The family $\gamma_{(A, \sigma)}^{V}=\left\{\gamma_{(A, \sigma), X}^{V}\right\}_{X \in \mathcal{C}_{1}}$, where

$$
\gamma_{(A, \sigma), X}^{V}=d_{V}^{-1}
$$

is a half-braiding of $\mathcal{C}$ relative to $\mathcal{C}_{1}$. Set

$$
\varphi_{V}(A, \sigma)=\left(E_{(A, \sigma)}^{V}, \gamma_{(A, \sigma)}^{V}\right) \in \mathcal{Z}_{G}(\mathcal{C})
$$

For a morphism $f:(A, \sigma) \rightarrow(B, \rho)$ in $\mathcal{Z}_{G}(\mathcal{C})$, set

$$
\varphi_{V}(f)=V(B)
$$

The monoidal constraints of $\varphi_{V}$ are defined for any $(A, \sigma),(B, \rho) \in \mathcal{Z}_{G}(\mathcal{C})$ by

$$
\left(\varphi_{V}\right)_{2}((A, \sigma),(B, \rho))=\text { and }\left(\varphi_{V}\right)_{0}=
$$

Then $\left(\varphi_{V},\left(\varphi_{V}\right)_{2},\left(\varphi_{V}\right)_{0}\right)$ is a pivotal strong monoidal $\mathbb{k}$-linear endofunctor of $\mathcal{Z}_{G}(\mathcal{C})$ such that $\varphi_{V}\left(\mathcal{Z}_{\beta}(\mathcal{C})\right) \subset \mathcal{Z}_{|V|^{-1} \beta|V|}(\mathcal{C})$ for all $\beta \in G$.

The endofunctors $\left\{\varphi_{V}\right\}_{V \in \mathcal{E}}$ are related as follows. First, pick any $U \in \mathcal{E}_{\alpha}$, $V \in \mathcal{E}_{\beta}, W \in \mathcal{E}_{\beta \alpha}$ with $\alpha, \beta \in G$. For any $(A, \sigma) \in \mathcal{Z}_{G}(\mathcal{C})$, set

$$
\zeta_{(A, \sigma)}^{U, V, W}=d_{U}^{-1} d_{V}^{-1}
$$


Then the family $\zeta^{U, V, W}=\left\{\zeta_{(A, \sigma)}^{U, V, W}\right\}_{(A, \sigma) \in \mathcal{Z}_{G}(\mathcal{C})}$ is a monoidal natural isomorphism from $\varphi_{U} \varphi_{V}$ to $\varphi_{W}$. Second, pick any $U \in \mathcal{E}_{1}$. For any $(A, \sigma) \in \mathcal{Z}_{G}(\mathcal{C})$, set

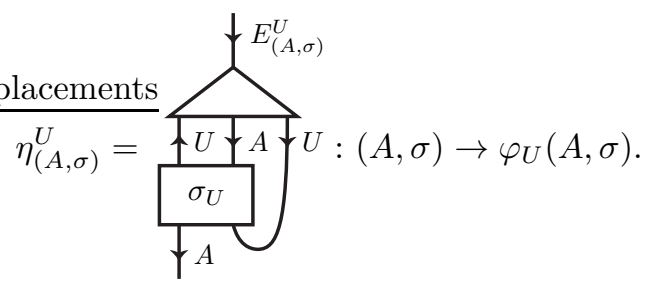

Then the family $\eta^{U}=\left\{\eta_{(A, \sigma)}^{U}\right\}_{(A, \sigma) \in \mathcal{Z}_{G}(\mathcal{C})}$ is a monoidal natural isomorphism from the identity endofunctor $1_{\mathcal{Z}_{G}(\mathcal{C})}$ to $\varphi_{U}$.

Step 2. For $U, V \in \mathcal{E}_{\alpha}$ with $\alpha \in G$, the family $\delta^{U, V}=\left\{\delta_{(A, \sigma)}^{U, V}\right\}_{(A, \sigma) \in \mathcal{Z}_{G}(\mathcal{C})}$, where

$$
\delta_{(A, \sigma)}^{U, V}=d_{V}^{-1}
$$

is a monoidal natural isomorphism from $\varphi_{U}$ to $\varphi_{V}$. These isomorphisms are related as follows: for any $U, V, W \in \mathcal{E}_{\alpha}$,

$$
\delta^{U, V} \delta^{V, W}=\delta^{U, W} \quad \text { and } \quad \delta^{U, U}=\operatorname{id}_{\varphi_{U}} .
$$

Step 3. For $\alpha \in G$, the family $\left(\varphi_{V}, \delta^{U, V}\right)_{U, V \in \mathcal{E}_{\alpha}}$ is a projective system in the category of pivotal strong monoidal $\mathbb{k}$-linear endofunctors of $\mathcal{Z}_{G}(\mathcal{C})$. Since all $\delta^{U, V}$ 's are isomorphisms, this system has a well-defined projective limit

$$
\varphi_{\alpha}=\lim _{\longleftarrow}\left(\varphi_{V}, \delta^{U, V}\right)_{U, V \in \mathcal{E}_{\alpha}}
$$

which is a pivotal strong monoidal $\mathbb{k}$-linear endofunctor of $\mathcal{Z}_{G}(\mathcal{C})$. We can assume that $\varphi_{\alpha}\left(\mathcal{Z}_{\beta}(\mathcal{C})\right) \subset \mathcal{Z}_{\alpha^{-1} \beta \alpha}(\mathcal{C})$ for all $\beta \in G$. Denote by $\iota^{\alpha}=\left\{\iota_{V}^{\alpha}\right\}_{V \in \mathcal{E}_{\alpha}}$ the universal cone associated with the projective limit above: for $V \in \mathcal{E}_{\alpha}$,

$$
\iota_{V}^{\alpha}=\left\{\left(\iota_{V}^{\alpha}\right)_{(A, \sigma)}: \varphi_{\alpha}(A, \sigma) \rightarrow \varphi_{V}(A, \sigma)\right\}_{(A, \sigma) \in \mathcal{Z}_{G}(\mathcal{C})}
$$

is a monoidal natural isomorphism from $\varphi_{\alpha}$ to $\varphi_{V}$.

The transformations $\zeta$ and $\eta$ from Step 1 induce monoidal natural isomorphisms $\varphi_{2}(\alpha, \beta): \varphi_{\alpha} \varphi_{\beta} \rightarrow \varphi_{\beta \alpha}$ and $\varphi_{0}: 1_{\mathcal{Z}_{G}(\mathcal{C})} \rightarrow \varphi_{1}$, respectively. These isomorphisms are related to the universal cone as follows: for $U \in \mathcal{E}_{\alpha}, V \in \mathcal{E}_{\beta}, W \in \mathcal{E}_{\beta \alpha}$, and $R \in \mathcal{E}_{1}$, the following diagrams commute:
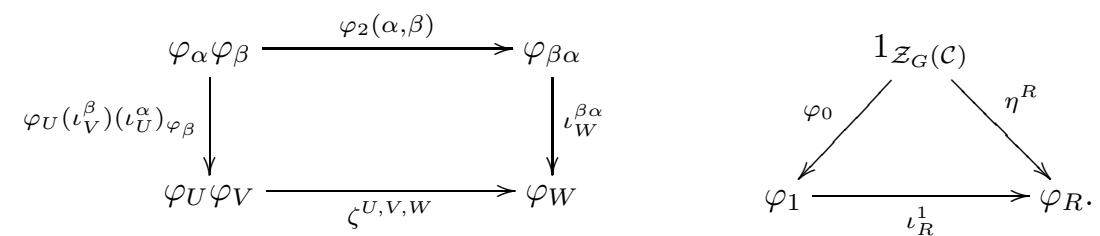

Note that $\varphi_{2}$ and $\varphi_{0}$ induce natural isomorphisms $\varphi_{\alpha} \varphi_{\alpha^{-1}} \simeq \varphi_{1} \simeq 1_{\mathcal{Z}_{G}(\mathcal{C})}$ and $\varphi_{\alpha^{-1}} \varphi_{\alpha} \simeq \varphi_{1} \simeq 1_{\mathcal{Z}_{G}(\mathcal{C})}$ for $\alpha \in G$. Hence, the endofunctor $\varphi_{\alpha}$ of $\mathcal{Z}_{G}(\mathcal{C})$ is an equivalence. Therefore

$$
\varphi=\left(\varphi, \varphi_{2}, \varphi_{0}\right): \bar{G} \rightarrow \operatorname{Aut}\left(\mathcal{Z}_{G}(\mathcal{C})\right), \alpha \mapsto \varphi_{\alpha}
$$


is a strong monoidal functor such that $\varphi_{\alpha}\left(\mathcal{Z}_{\beta}(\mathcal{C})\right) \subset \mathcal{Z}_{\alpha^{-1} \beta \alpha}(\mathcal{C})$ for all $\alpha, \beta \in G$. This is the crossing of $\mathcal{Z}_{G}(\mathcal{C})$.

A.4. The enhanced $G$-braiding. For $V \in \mathcal{E},(A, \sigma) \in \mathcal{Z}_{G}(\mathcal{C})$, and $X \in \mathcal{C}_{\text {hom }}$, set

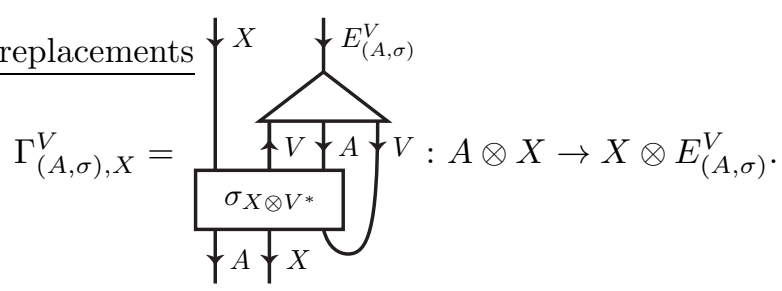

This is an isomorphism which is natural in $(A, \sigma)$ and in $X$. Also, for any $U, V \in \mathcal{E}$ with $|U|=|V|,(A, \sigma) \in \mathcal{Z}_{G}(\mathcal{C})$, and $X \in \mathcal{C}_{\text {hom }}$, the following diagram commutes:

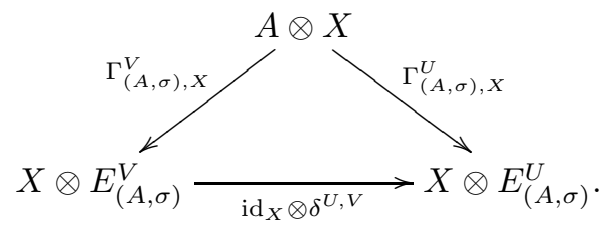

Then the transformation $\Gamma$ induces a family of isomorphisms

$$
\tau=\left\{\tau_{(A, \sigma), X}: A \otimes X \rightarrow X \otimes \mathcal{U}\left(\varphi_{|X|}(A, \sigma)\right)\right\}_{(A, \sigma) \in \mathcal{Z}_{G}(\mathcal{C}), X \in \mathcal{C}_{\text {hom }}}
$$

which is natural in $(A, \sigma)$ and in $X$, where $\mathcal{U}: \mathcal{Z}_{G}(\mathcal{C}) \rightarrow \mathcal{C}$ is the forgetful functor. The family $\tau$ is related to the universal cones $\left\{\iota^{\alpha}\right\}_{\alpha \in G}$ associated with $\varphi$ as follows: for any $(A, \sigma) \in \mathcal{Z}_{G}(\mathcal{C}), X \in \mathcal{C}_{\text {hom }}$, and $V \in \mathcal{E}_{|X|}$,

$$
\left(\operatorname{id}_{X} \otimes\left(\iota_{V}^{|X|}\right)_{(A, \sigma)}\right) \tau_{(A, \sigma), X}=\Gamma_{(A, \sigma), X}^{V}
$$

We call the family $\tau$ the enhanced $G$-braiding in $\mathcal{Z}_{G}(\mathcal{C})$. The enhanced $G$-braiding satisfies properties generalizing that of a $G$-braiding. In particular, it is distributive in each variable with respect to the monoidal product: for all $(A, \sigma),(B, \rho) \in \mathcal{Z}_{G}(\mathcal{C})$ and all $X, Y \in \mathcal{C}_{\text {hom }}$,

$$
\tau_{(A, \sigma), X \otimes Y}=\left(\operatorname{id}_{X \otimes Y} \otimes \varphi_{2}(|Y|,|X|)_{(A, \sigma)}\right)\left(\operatorname{id}_{X} \otimes \tau_{\varphi_{|X|}(A, \sigma), Y}\right)\left(\tau_{(A, \sigma), X} \otimes \operatorname{id}_{Y}\right)
$$

and

$$
\begin{gathered}
\tau_{(A, \sigma) \otimes(B, \rho), X}=\left(\operatorname{id}_{X} \otimes\left(\varphi_{|X|}\right)_{2}((A, \sigma),(B, \rho))\right)\left(\tau_{(A, \sigma), X} \otimes \operatorname{id}_{\varphi_{|X|}(B, \rho)}\right) \circ \\
\circ\left(\operatorname{id}_{A} \otimes \tau_{(B, \rho), X}\right) .
\end{gathered}
$$

A.5. The $G$-braiding. The $G$-braiding in $\mathcal{Z}_{G}(\mathcal{C})$ is induced from the enhanced $G$-braiding. More precisely, for all $(A, \sigma) \in \mathcal{Z}_{G}(\mathcal{C})$ and $(B, \rho) \in \mathcal{Z}_{G}(\mathcal{C})_{\text {hom }}$,

$$
\tau_{(A, \sigma),(B, \rho)}=\tau_{(A, \sigma), B}:(A, \sigma) \otimes(B, \rho) \rightarrow(B, \rho) \otimes \varphi_{|(B, \rho)|}(A, \sigma) .
$$

is a morphism in $\mathcal{Z}_{G}(\mathcal{C})$. Then the family

$$
\left\{\tau_{(A, \sigma),(B, \rho)}\right\}_{(A, \sigma) \in \mathcal{Z}_{G}(\mathcal{C}),(B, \rho) \in \mathcal{Z}_{G}(\mathcal{C})_{\text {hom }}}
$$

is a $G$-braiding in $\mathcal{Z}_{G}(\mathcal{C})$. In particular, $\mathcal{Z}_{G}(\mathcal{C})$ is a $G$-braided category. 
A.6. Ribboness of the $G$-center. The twist $\theta$ of $\mathcal{Z}_{G}(\mathcal{C})$ is computed as follows: if $(A, \sigma) \in \mathcal{Z}_{\alpha}(\mathcal{C})$ with $\alpha \in G$, then for any $U \in \mathcal{E}_{\alpha}$,

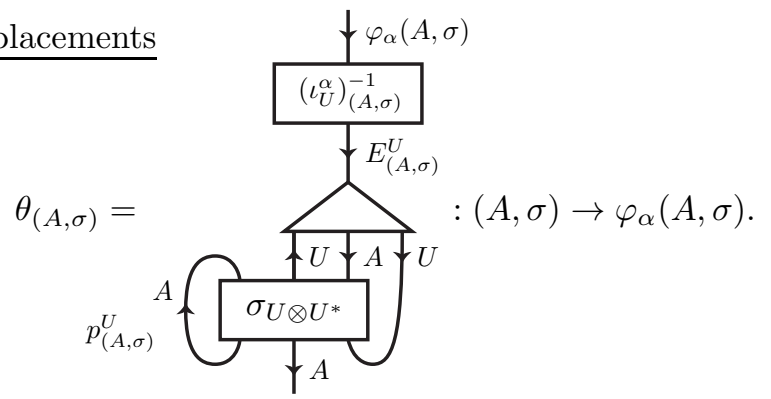

Recall that $\mathcal{Z}_{G}(\mathcal{C})$ is $G$-ribbon if the twist $\theta$ is self-dual (see Section 2.5). By TV3, Lemma 6.1 , a necessary and sufficient condition for $\mathcal{Z}_{G}(\mathcal{C})$ to be $G$-ribbon is that

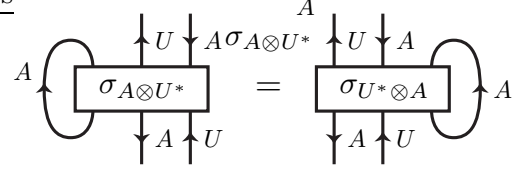

for all $\alpha \in G,(A, \sigma) \in \mathcal{Z}_{\alpha}(\mathcal{C})$, and $U \in \mathcal{E}_{\alpha}$.

\section{REFERENCES}

[Ba] Balsam, B., Turaev-Viro invariants as an extended TQFT III, arXiv:1012.0560.

[EGNO] Etingof, P., Gelaki, S., Nikshych, D., Ostrik, V., Tensor categories, Mathematical Surveys and Monographs, 205. American Mathematical Society, Providence, RI, 2015.

[GNN] Gelaki, S., Naidu, D., Nikshych, D., Centers of fusion categories, Alg. Number Theory 3 (2009), 959-990.

[ML] Mac Lane, S., Categories for the working mathematician, second ed., Springer-Verlag, New York, 1998.

[Mu Müger, M., From subfactors to categories and topology. II. The quantum double of tensor categories and subfactors, J. Pure Appl. Algebra 180 (2003), 159-219.

[SW] Schweigert, C., Woike, L., Extended homotopy quantum field theories and their orbifoldization, J. Pure Appl. Algebra 224 (2020).

[Tu1] Turaev, V., Quantum invariants of knots and 3-manifolds, de Gruyter Studies in Mathematics, 18. Walter de Gruyter, Berlin, 1994.

[Tu2] Turaev, V., Homotopy Quantum Field Theory, EMS Tracts in Math. 10, European Math. Soc. Publ. House, Zürich 2010.

[TV1] Turaev, V., Virelizier, A., On two approaches to 3-dimensional TQFTs, arXiv:1006.3501.

[TV2] Turaev, V., Virelizier, A., On 3-dimensional homotopy quantum field theory, I, Internat. J. Math. 23 (2012), no. 9, 1250094, 28 pp.

[TV3] Turaev, V., Virelizier, A., On the graded center of graded categories, J. Pure Appl. Algebra 217 (2013), no. 10, 1895-1941.

[TV4] Turaev, V., Virelizier, A., On 3-dimensional homotopy quantum field theory II: The surgery approach, Internat. J. Math. 25 (2014), no. 4, 1450027, 66 pp.

[TV5] Turaev, V., Virelizier, A., Monoidal Categories and Topological Field Theory, Progress in Mathematics, 322. Birkhäuser, Basel, 2017. xii+523 pp. 


\author{
Vladimir Turaev \\ Department of Mathematics, Indiana University \\ BloOMingTON IN47405 USA \\ AND \\ IRMA, 7 RUE RENE DESCARTES \\ 67084 Strasbourg France \\ Alexis Virelizier \\ Université de LiLle \\ Laboratoire Paul Painlevé (CNRS, UMR 8524) \\ F-59000 LiLle France \\ E-MAIL: ALEXIS.VIRELIZIER@UNIV-LILLE.FR
}

